\title{
, Non-Coherent Successive Relaying and Cooperation: Principles, Designs, and Applications
}

\author{
Li Li, H. Vincent Poor, Fellow, IEEE, and Lajos Hanzo, Fellow, IEEE
}

\begin{abstract}
4 Abstract-Cooperative communication is capable of forming 5 a virtual antenna array for each node (user) in a network by 6 allowing the nodes (users) to relay the messages of others to the 7 destination. Such a relay aided network may be viewed as a dis8 tributed multiple-input multiple-output (MIMO) system relying 9 on the spatially distributed single antennas of the cooperating 10 mobiles, which avoids the correlation of the antenna elements 11 routinely encountered in conventional MIMO systems and hence 12 attains the maximum achievable diversity gain. Therefore, the 13 family of cooperative communication techniques may be regarded 14 as a potential solution for future wireless networks. However, 15 constrained by the half-duplex transmit/receive mode of most 16 practical transceivers, the cooperative networks may impose a 17 severe $50 \%$ throughput loss. As a remedy, successive relaying can 18 be employed, which is capable of mimicking a full-duplex relay 19 and thereby recovering much of the $\mathbf{5 0 \%}$ throughput loss. Fur20 thermore, for the sake of bypassing power-hungry and potentially 21 excessive-complexity channel estimation, noncoherent detection 22 techniques may be employed for multiple-antenna aided systems, 23 because estimating all the associated channels may become un24 realistic. Explicitly, the mobile-stations acting as relays cannot 25 be realistically expected to estimate the source-to-relay channels. 26 To motivate further research on noncoherent successive relay27 ing aided systems, a comprehensive review of its basic concepts, 28 fundamental principles, practical transceiver designs and open 29 challenges is provided.
\end{abstract}

AQ1 30 Index Terms-Author, please supply index terms/keywords 31 for your paper. To download the IEEE Taxonomy go to 32 http://www.ieee.org/documents/taxonomy_v101.pdf.

\section{INTRODUCTION}

35

36 ONCEIVING high-quality wireless solutions in support of the wireless Internet is of paramount importance. 37 Achieving a low bit-error-rate (BER), high system throughput, 38 low complexity, low delay, as well as seamless connectivity 39 across the entire coverage area are just a few of the critical

Manuscript received April 2, 2014; revised September 6, 2014, November 27, 2014, and February 19, 2015; accepted April 5, 2015. The financial support of the European Union under the auspices of the Concerto project, and that of the RC-UK under the auspices of the India-UK Advanced Technology Centre known as In-ATC, and that of the European Research Council's Advanced Fellow Grant, as well as that of the U.S. National Science Foundation under Grants CNS-1456793 and ECCS-1343210 is gratefully acknowledged.

L. Li is with the Provincial Key Lab of Information Coding and Transmission, Southwest Jiaotong University, Chengdu 610031, China. He is also with the School of Electronics and Computer Science (ECS), University of Southampton, SO17 1BJ, U.K. (e-mail: 111d13@ecs.soton.ac.uk).

H. V. Poor is with the School of Engineering and Applied Science, Princeton University, Princeton, NJ 08544 USA (e-mail: poor@ princeton.edu).

L. Hanzo is with the School of Electronics and Computer Science (ECS), University of Southampton, SO17 1BJ Southampton, U.K. (e-mail: lh@ecs.soton.ac.uk).

Digital Object Identifier 10.1109/COMST.2015.2424157 design concerns in contemporary wireless systems. It is an- 40 ticipated that wireless tele-traffic will grow substantially over 41 the forthcoming ten years [1, Figure 1]. Based on this trend, 42 techniques that are capable of improving system capacity are 43 of salient significance. In this context, multiple-input multiple- 44 output (MIMO) wireless systems have attracted considerable 45 attention in recent years [2]-[7], because they exhibit a capacity 46 that increases linearly with the transmit power, provided that the 47 number of MIMO elements can also be linearly increased. 48

However, the MIMO antenna elements have to be sufficiently 49 far apart to experience independent fading, which may be 50 impractical in the uplink owing to the limited size of the mobile 51 handset. Furthermore, even a downlink MIMO base station 52 (BS) transmitter associated with a relatively large element 53 separation may not benefit from independent fading, when it 54 is subjected to shadow-fading imposed for example by large- 55 bodied vehicles or other local shadowing objects [8]. 56

As a remedy, cooperative communications is capable of 57 forming a virtual antenna array (VAA) for each node (user) 58 in a cooperative network by allowing the nodes (users) to 59 relay the messages of other's to the destination. Hence, such a 60 relay aided network practically constitutes a distributed MIMO 61 system relying on the spatially distributed single antennas of the 62 mobiles. This allow us to avoid the correlation of the antenna 63 elements that arises in conventional MIMO systems.

The germination of the basic idea of cooperative commu- 65 nication can be traced back to the concept of the relay chan- 66 nel, which was devised by Van der Meulen in [9] and was 67 later characterized from an information-theoretic perspective 68 by Cover and El Gamal in [10]. Basic relaying protocols were 69 also proposed in [10]. To elaborate a little further, cooperative 70 communications benefits from the broadcast nature of wireless 71 transmitters, which allows the relays to receive and retransmit 72 all signals, leading to the concept of "cooperative diversity". 73 Laneman et al. characterised both the decode-and-forward (DF) 74 and amplify-and-forward (AF) protocols in [11] and [12] by 75 evaluating both their diversity orders and their outage probabil- 76 ities. Similar concepts were investigated also by Sendonaris et 77 $a l$. in [13] and [14]. These seminal paradigms [11]-[14] have 78 attracted substantial research attention and inspired a number 79 of novel contributions in the research area of cooperative 80 communications. Some of the important milestones achieved 81 in the early stages of cooperative communications research are 82 summarized in Table I.

However, despite the above-mentioned benefits, cooperation 84 techniques impose their own problems as well. Since conven- 85 tional transceivers cannot transmit and receive at the same time, 86 we have to allocate two orthogonal channels for the reception 87 
TABLE I

BRIEF History OF EARLY INVESTIGATIONS OF COOPERATIVE COMMUNICATIONS

\begin{tabular}{|c|c|c|}
\hline Year & Authors & Contributions \\
\hline 1971 & Van der Meulen [9] & $\begin{array}{l}\text { Heralded the concept of cooperative communication and established the basic } \\
\text { three-terminal relaying model. }\end{array}$ \\
\hline 1979 & Cover and El Gamal [10] & $\begin{array}{l}\text { Analysed the capacity of the three-terminal based relaying system along with } \\
\text { proposing some basic relaying protocols. }\end{array}$ \\
\hline 2003 & Sendonaris et al. [13], [14] & \multirow{2}{*}{$\begin{array}{l}\text { Evaluated the performance of several classical cooperative protocols. The } \\
\text { remarkable advantages of cooperative communication were quantified in terms of } \\
\text { their large power savings, low outage probability and high diversity order. }\end{array}$} \\
\hline 2004 & Laneman et al. [12] & \\
\hline \multirow[b]{2}{*}{2005} & Azarian et al. [15] & $\begin{array}{l}\text { Investigated the achievable diversity-multiplexing trade-off (DMT) of the } \\
\text { cooperative network. }\end{array}$ \\
\hline & $\begin{array}{l}\text { Høst-Madsen and Zhang } \\
{[16]}\end{array}$ & $\begin{array}{l}\text { Optimum resource allocation was conceived for cooperative systems, which is } \\
\text { capable of improving both the energy efficiency and battery recharge-period of } \\
\text { cooperative communications. }\end{array}$ \\
\hline 2006 & Bletsas et al. [17] & Relay selection regimes were exploited for improving the outage performance. \\
\hline
\end{tabular}

88 and transmission at the relay node $(\mathrm{RN})$. Consequently, in the 89 conventional cooperative protocols, such as in AF, DF as well as 90 compress-and-forward [18] protocols, the holistic transmission 91 of an information stream has to be split into two phases, 92 namely the "broadcast phase" for the RN's reception and the 93 "cooperative phase" for the RN's transmission. By contrast, 94 the direct-transmission of the information stream dispensing 95 with relaying requires only a single phase. Therefore, the 96 conventional cooperation techniques actually impose a severe $9750 \%$ throughput loss, which goes against the demand for higher 98 wireless data rates, as highlighted in [1] and [19].

99 For the sake of recovering the throughput loss incurred 100 in conventional half-duplex (HD) relaying based cooperation 101 systems, while retaining their substantial benefits of extending 102 the radio coverage, a range of advanced techniques have been 103 proposed in recent years, which may be briefly categorised as 104 follows:

105 (a) Switching to full-duplex (FD) relaying mode-In the 106 early investigations of relaying techniques [9], [10], the ide107 alized FD relaying mode was assumed for analysing the ca108 pacity upper-bound of relaying channels. However, from a 109 practical perspective, relying on FD relaying may be infeasible 110 for compact single-antenna aided relays. Since a high-power 111 interfering signal will be fed back to the RN's input from the 112 RN's output, this results in the so-called "self-interference" (SI) 113 problem. Hence, instead of the FD mode, the HD mode has 114 been employed in the seminal contributions on user cooperation 115 [11]-[14], [20].

116 Nonetheless, with recent developments in digital signal pro117 cessing techniques, mitigating the unwanted SI in the feedback 118 path between the transmit antenna and receive antenna becomes 119 possible. The related early results [21]-[23] were focused on 120 realizing single-input single-output (SISO) repeaters. Later, 121 the FD transceiver design philosophy was extended to multi122 antenna aided spatial suppression techniques, which invoked 123 more sophisticated adaptive filter algorithms [24]-[26]. Most 124 recently, Katti et al. at Stanford made substantial progress in 125 terms of building in-band full-duplex radios. Explicitly, with 126 the aid of antenna cancellation techniques, they implemented 127 in-band full-duplex transceivers [27]-[29], which are capable of 128 reducing the SI to the noise floor. Motivated by the development 129 of FD transceiver techniques, the theoretical analysis of FD

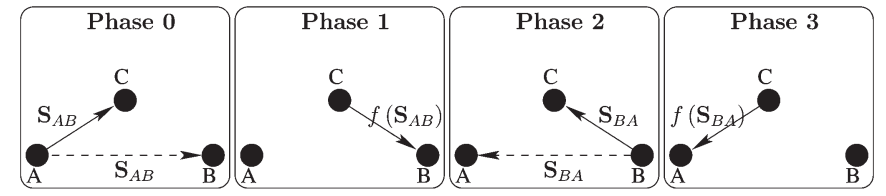

(a)

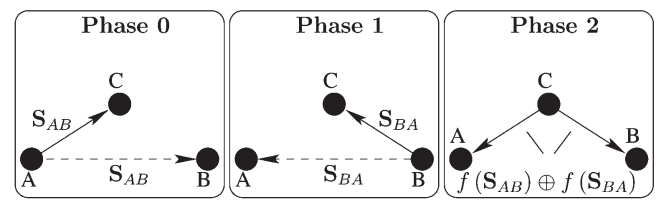

(b)

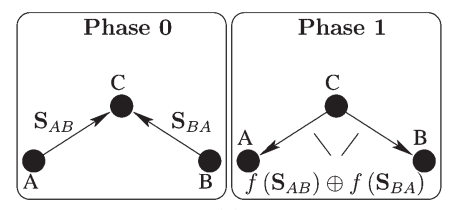

(c)

Fig. 1. The transmission arrangements of conventional DF relaying, threestep two-way relaying, and two-step two-way relaying while exchanging the information sequences $f S_{A B}$ and $\mathbf{S}_{B A}$ between node $A$ and $B$ with the aid of the relay node $C$, where $f\left(\mathbf{S}_{A B}\right)$ denotes the estimate of $\mathbf{S}_{A B}$.

relaying based cooperative networks enjoyed a renaissance in 130 [30]-[32], where the impact of SI imposed by practical FD 131 relays was taken into account.

However, as stated above, most of the proposed FD relays 133 have to employ spatially separated transmit and receive anten- 134 nas, which should have a sufficiently high physical isolation, 135 whilst additionally relying on sophisticated adaptive filter algo- 136 rithms. Hence, the implementation of FD relays still remains 137 an open challenge at the time of writing and this motivates the 138 design of alternative solutions.

(b) Devising sophisticated relaying protocols-For the par- 140 ticular scenario in which two nodes communicate with each 141 other with the aid of a RN, "two-way" relaying was devised in 142 [33]-[35], which has attracted considerable attention in recent 143 years [36]-[40], since it inherently accommodates bandwidth- 144 efficient network coding techniques. When exchanging the 145 pair of information sequences $\mathbf{S}_{A B}$ and $\mathbf{S}_{B A}$ between node 146 $A$ and node $B$ with the aid of the RN $C$, the transmission 147 arrangements of conventional DF relaying and of two-way 148 


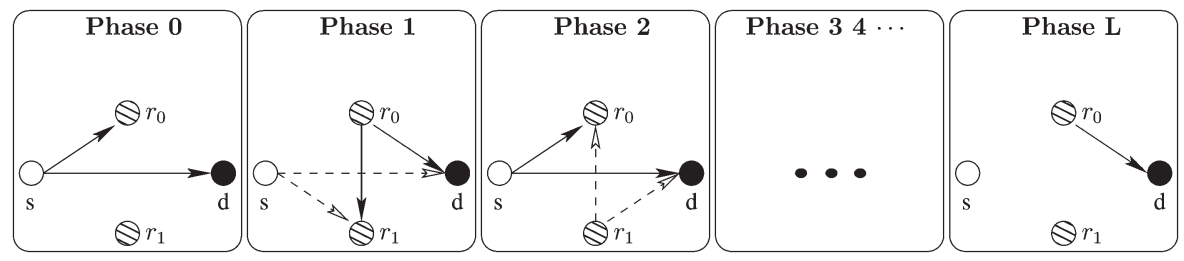

Fig. 2. Transmission schedule of the successive relaying protocol.

149 relaying are contrasted in Fig. 1. Observe in Fig. 1 that the 150 four distinct transmission phases required by conventional DF 151 relaying are compressed into three phases in the "three-step 152 two-way relaying" regime or are further compressed into two 153 phases in "two-step two-way relaying" regime. Hence the 50\% 154 throughput loss incurred by conventional relaying protocols is 155 mitigated. In more detail,

1) Three-step two-way relaying: During Phase 0 , the $\mathrm{SN}$ A broadcasts its information sequence $\mathbf{S}_{A B}$, while the RN C generates an estimate of $\mathbf{S}_{A B}$, namely $f\left(\mathbf{S}_{A B}\right)$ relying on the signal sequence received. ${ }^{1}$ Meanwhile, the DN B also obtains a copy of the information sequence $\mathbf{S}_{A B}$, which however may be contaminated both by the channel's fading effects and by additive white Gaussian noise (AWGN). Then, a similar process will be invoked again during the forthcoming Phase 1. This time, the SN becomes the receiver, the DN becomes the transmitter and the broadcast information sequence becomes $\mathbf{S}_{B A}$. As seen in sub-figure "(a) Conventional DF relaying" of Fig. 1, the RN C directly forwards the estimate $f\left(\mathbf{S}_{A B}\right)$ or $f\left(\mathbf{S}_{B A}\right)$ during its cooperative phase, which only bears the information of $\mathbf{S}_{A B}$ or $\mathbf{S}_{B A}$. By contrast, the RN $\mathrm{C}$ forwards the XOR of $f\left(\mathbf{S}_{A B}\right)$ and $f\left(\mathbf{S}_{B A}\right)$ i.e. $f\left(\mathbf{S}_{A B}\right) \oplus f\left(\mathbf{S}_{B A}\right)$ during Phase 2 of the 3-step two-way relaying, which bears both the information of $\mathbf{S}_{A B}$ and that of $\mathbf{S}_{B A}$. Naturally, each node A and node B knows its own message $\mathbf{S}_{A B}$ or $\mathbf{S}_{B A}$. Hence each can readily extract the remote message by subtracting its own message from $f\left(\mathbf{S}_{A B}\right) \oplus f\left(\mathbf{S}_{B A}\right)$. For example, assuming that perfect detection is achieved at the $\mathrm{RN} \mathrm{C}$, as well as that we have $\mathbf{S}_{A B}=\underbrace{0101}_{\mathbf{S}_{A B}}, \mathbf{S}_{B A}=\underbrace{1100}_{\mathbf{S}_{B A}}$, we readily see that $f\left(\mathbf{S}_{A B}\right) \oplus f\left(\mathbf{S}_{B A}\right)=\underbrace{1001}_{\text {XOR }}$. Hence, during Phase 2, node A becomes capable of recovering the remote message of $\mathbf{S}_{B A}$ by calculating $\underbrace{1100}_{\mathbf{S}_{B A}}=\underbrace{0101}_{\mathbf{S}_{A B}} \oplus \underbrace{1001}_{\text {XOR }}$. Practically, the XOR of $f\left(\mathbf{S}_{A B}\right)$ and $f\left(\mathbf{S}_{B A}\right)$ received at node A may also be contaminated both by the channel's fading effects and by the AGWN. Fortunately, as stated earlier, node A has another copy of its remote message, which was received during Phase 0 . Hence a joint detection of the remote message based on both the signal received during Phase 0 and on that received during Phase 2 may be implemented at node A. Consequently, a diversity gain is obtained.

${ }^{1}$ Hence, the decode-and-forward protocol is adopted at the RN C.
2) Two-step two-way relaying: During Phase 0, node A 193 and node B simultaneously broadcast their information 194 sequences $\mathbf{S}_{A B}$ and $\mathbf{S}_{B A}$. Limited by the HD transceiver 195 architecture, neither node A nor node B can receive any 196 signal during this phase. Meanwhile, the RN C receives a 197 composite signal, which consists of the $\mathbf{S}_{A B}$ component 198 and of the $\mathbf{S}_{B A}$ component. For the sake of calculating 199 the XOR of $\mathbf{S}_{A B}$ and $\mathbf{S}_{B A}$, the RN C has to be able to 200 detect each of the information sequences $\mathbf{S}_{A B}$ and $\mathbf{S}_{B A} 201$ from their composite. ${ }^{2}$ Since the information sequences 202 $\mathbf{S}_{A B}$ and $\mathbf{S}_{B A}$ interfere with each other at the RN C, 203 interference cancellation has to be invoked at the RN C. 204 Similar to the last phase of three-step two-way relaying, 205 during Phase 1 of two-step two-way relaying, the RN C 206 forwards the XOR of $f\left(\mathbf{S}_{A B}\right)$ and $f\left(\mathbf{S}_{B A}\right)$ to the DN A 207 and B. Then, the DN A (B) extracts its own information 208 sequence $\mathbf{S}_{A B}\left(\mathbf{S}_{B A}\right)$ from $f\left(\mathbf{S}_{A B}\right) \oplus f\left(\mathbf{S}_{B A}\right)$ for the 209 sake of decoding the information sequence transmitted 210 by the remote node B (A). However, node A (B) re- 211 ceives only a single copy of the remote message during 212 the two-step scheme, because the direct link between A 213 and B becomes unavailable in Phase 0 of the two-step 214 scheme owing to the HD limitation. Hence, compared to 215 the three-step scheme, the two-step scheme completely 216 recovers the throughput loss at the cost of degrading the 217 achievable diversity gain.

However, the application scenario of the two-way relaying 219 scheme is constrained to bidirectional communications.

(c) Mimicking the FD relaying mode despite relying on 221 using HD relays - A novel relaying protocol, namely succes- 222 sive relaying or two-path relaying was conceived in [43], which 223 is capable of mimicking a FD relay by relying on a pair of 224 HD relays, where the two HD relays alternately serve as the 225 transmitter and receiver of the virtual FD relay. Based on [43], 226 as well as inspired by the related benefits reported in [33], [34], 227 [44], and [45], a generalized successive relaying protocol was 228 proposed by Fan et al. in [46], offering further insights both 229 into the achievable rates and into the associated diversity versus 230 multiplexing trade-off.

The transmission arrangement of the successive relaying pro- 232 tocol proposed in [46] is illustrated in Fig. 2, where we observe 233 $(L+1)$ processing phases. To elaborate a little further, observe 234 in Fig. 2 that the SN $s$ constantly transmits information during 235

\footnotetext{
${ }^{2}$ In some studies, the DF protocol employed at the $\mathrm{RN} \mathrm{C}$ is replaced by the AF protocol [41] or by the denoise-and-forward protocol [42]. Hence interference suppression for detecting each of the information sequences $\mathbf{S}_{A B}$ and $\mathbf{S}_{B A}$ at the $\mathrm{RN}$ is circumvented. Instead, the RN C may only amplify the composite signal received and forward it to node $\mathrm{A}$ and node $\mathrm{B}$.
} 
TABLE II

HISTORY OF SUCCESSIVE RELAYING

\begin{tabular}{|c|c|c|}
\hline Year & Authors & Contributions \\
\hline 2004 & Oechtering and Sezgin [43] & $\begin{array}{l}\text { The concept of successive relaying was introduced, where a new cooperative } \\
\text { transmission scheme was conceived. }\end{array}$ \\
\hline 2005 & nd Wittnebe & \multirow{3}{*}{$\begin{array}{l}\text { The benefits of employing successive relaying with regard to conventional } \\
\text { relaying, especially the higher achievable throughput and the better } \\
\text { diversity-multiplexing trade-off were reported. }\end{array}$} \\
\hline 2006 & Belfiore & \\
\hline \multirow{3}{*}{2007} & Yang & \\
\hline & Rankov and Wittneben [34] & \multirow{2}{*}{$\begin{array}{l}\text { A generalized successive relaying protocol was proposed, offering further } \\
\text { insights both into the achievable rates and into the associated } \\
\text { diversity-multiplexing trade-off. The successive relaying induced interference } \\
\text { problem was also mentioned. }\end{array}$} \\
\hline & Fan & \\
\hline 2009 & Wicaksana et al. [49] & \multirow{2}{*}{$\begin{array}{l}\text { Techniques for suppressing the successive relaying induced interference were } \\
\text { proposed under the idealized assumption that perfect channel state information } \\
\text { (CSI) was available at the receiver. }\end{array}$} \\
\hline \multirow[b]{2}{*}{2011} & Luo et al. [48] & \\
\hline & Tian et al. [50] & $\begin{array}{l}\text { A sophisticated combination of relay selection, distributed space-time block } \\
\text { coding as well as adaptive relaying schemes was proposed for the sake of } \\
\text { overcoming the inter-relay interference of the successive relaying regime. }\end{array}$ \\
\hline
\end{tabular}

TABLE III

Three Potential Solutions for Mitigating the 50\% Throughrut Loss Incurred in Conventional Relaying Protocols

\begin{tabular}{|l|r|r|r|}
\hline Solutions: & Full-Duplex Relaying & Two-Way Relaying & Successive Relaying \\
\hline Challenges: & Self-Interference & Co-Channel Interference & Inter-Relay Interference \\
\hline Limitations: & Require infrastructure relays & Limited to bidirectional communications & Require an excessive number of relays \\
\hline
\end{tabular}

236 every phase, except for the last phase. Hence, in successive 237 relaying, a normalized throughput of $\frac{L}{L+1}$ is achieved, which 238 will get close to that of direct-transmission upon increasing 239 the number of processing phases $L$. Consequently, the $50 \%$ 240 throughput loss incurred in conventional relaying protocols 241 may be mitigated at the cost of increased delay. The specific 242 actions involved in every phase of Fig. 2 will be further detailed 243 in Section II-A. However, observe in Fig. 2 that increased 244 interference will be encountered both at the RNs and at the DN, 245 which is termed inter-relay interference (IRI) and co-channel 246 interference (CCI), respectively. To combat the successive re247 laying induced interference, a range of solutions were proposed 248 in [47]-[49]. Furthermore, the authors of [50] replace the repe249 tition of source symbols by the relays as in [46] with a simple 250 coding scheme for the sake of achieving full diversity by using 251 signal space diversity techniques. Further improvements based 252 on space-time block coding combined with coordinate inter253 leaving are derived in [51]. The effect of the successive relaying 254 induced interference will be discussed in detail in Section III-C. 255 The history of successive relaying is briefly summarized 256 in Table II.

257 The above-mentioned solutions conceived for recovering 258 the conventional relaying-induced 50\% throughput loss are 259 summarized in Table III, where the challenges they have to 260 overcome as well as the constraints they encounter are also 261 highlighted. Instead of assuming a fixed relaying infrastructure 262 based cooperative network, we rely on mobile relays consti263 tuted by idle mobile users, which is a more economical ap264 proach. Again, the above-mentioned two-way relaying protocol 265 is applicable only to bidirectional communications. By contrast, 266 the successive relaying approach is free from this constraint. 267 Hence successive relaying may be regarded as a more versatile 268 solution provided that its delay is tolerable. Hence, in this trea269 tise, we provide a detailed review of and tutorial on the design 270 of successive relaying protocols conceived for recovering the 271 50\% throughput loss of conventional HD relaying.
Furthermore, we will pay particular attention to the system's 272 coherent versus non-coherent (NC) detection strategy. 273

Classical coherent detection techniques rely on knowledge 274 of CSI for mitigating the deleterious effects of fading channels. 275 Practical channel estimation techniques typically rely on pilot 276 symbol assisted training techniques [52] and on the fact that in 277 general the consecutive channel impulse response (CIR) taps 278 are correlated in time, as governed by the normalized Doppler 279 frequency. However, an $M$-transmitter, $N$-receiver MIMO sys- 280 tem has to estimate $(M \times N)$ channels, which will substan- 281 tially increase the complexity of the entire system, especially 282 at high normalized Doppler frequencies. Furthermore, in the 283 specific scenario of a VAA-assisted cooperative network, it is 284 unrealistic to expect that in addition to the task of relaying, the 285 relay-station would dedicate further precious resources to the 286 estimation of the source-to-relay channel in support of coherent 287 detection. Based on these discussions, NC detection schemes 288 may be deemed to be promising solutions in the context of 289 cooperative networks, since they are capable of retaining the 290 cooperative diversity gain, while circumventing the potentially 291 excessive burden of multiple-antenna based channel estimation. 292

Numerous differential detection schemes have been devised 293 for noncoherent receivers. For AWGN channels, the conven- 294 tional differential detection (CDD) philosophy was further de- 295 veloped to multiple-symbol differential detection (MSDD) by 296 Divsalar et al. in [53] for the sake of reducing the BER per- 297 formance gap between the CDD of $M$-ary phase-shift keying 298 (MPSK) and the coherent detection of differentially encoded 299 MPSK. This performance gain over CDD is achieved by ex- 300 tending the observation interval from two adjacent symbols to 301 multiple consecutive symbols, while at the same time making 302 a joint decision on these multiple consecutive symbols. A fast 303 algorithm for MSDD was also proposed in [54]. However, 304 the main disadvantage of MSDD is its high complexity. By 305 contrast, Leib et al. [55] and Edbauer [56] proposed sim- 306 ple decision-feedback aided differential detection (DF-DD) 307 
308 techniques, which achieve an equally competitive performance 309 at a low computational complexity. Later, Adachi et al. [57] 310 demonstrated that these DF-DD techniques can be regarded 311 as an approximate realisation of MSDD that proposed by 312 Divsalar et al. in [53], which are capable of significantly reduce 313 the computational complexity at the cost of incurring a slight 314 degradation of the BER performance. ${ }^{3}$ For fading channels, es315 pecially in high-Doppler scenarios, the CDD typically exhibits 316 a high BER-floor. To reduce this error-floor, Divsalar et al. 317 [58] and Ho et al. [59] further improved the original MSDD 318 algorithm by taking into account the channel's correlation 319 matrix in the multiple-symbol detection metric. Similarly, to 320 mitigate the system's computational complexity, the DF-DD 321 technique was also extended to Rayleigh fading channels in 322 [60]. As a further milestone, the sphere decoding algorithm 323 advocated in [61] was incorporated into the MSDD algorithm 324 of [58] and [59] by Lampe et al. in [62], which resulted in 325 the conception of the multiple-symbol differential sphere detec326 tion (MSDSD) technique. The MSDSD algorithm substantially 327 reduces the complexity of the MSDD algorithm without any 328 BER degradation with respect to the MSDD algorithm [58]. 329 Hence it achieves a better trade-off between the attainable BER 330 performance and the associated decoding complexity and there331 fore attracted considerable attention. However, the decoding 332 complexity of the MSDSD algorithm imposed at low SNRs 333 still grows exponentially upon decreasing the SNR. For solving 334 this particular drawback of MSDSD, Pun et al. devised the 335 Multiple-Symbol Differential Detection based Fano-algorithm 336 (Fano-MSDD) in [63] and [64] by further developing Fano's 337 original algorithm [65] as an efficient MSDD receiver. On 338 the other hand, to transform the hard-decision-based (HDB) 339 MSDSD algorithm to a more energy-efficient, soft-decision340 based (SDB) iterative detection scheme, the MSDSD algorithm 341 was further developed into the soft-input soft-output MSDSD 342 (SISO-MSDSD) regime of [66].

343 For the sake of supporting the operation of cooperative 344 systems, the noncoherent detection algorithms employed at the 345 DN should become capable of simultaneously processing the 346 multiple input signal sequences received via both the source347 to-destination link and the relay-to-destination link. More 348 explicitly, as a benefit of cooperative diversity, the DN of a 349 cooperative network will have to combine multiple versions 350 of the same codeword transmitted via both the source-to351 destination link and relay-to-destination links. For the sake of 352 satisfying this requirement, the MSDSD algorithm of [62] was 353 further modified by Wang et al. in [67] and [68]. We will 354 refer to this variant of the MSDSD algorithm as the "multiple355 path processing based MSDSD". Consequently, the application 356 scenario of the MSDSD algorithm was extended from single357 link direct transmission based systems to cooperative systems. 358 Motivated by [67] and [68], the single-user/single-path MSDSD 359 algorithm of [62] was further developed into a novel relay-aided 360 form in [69]. Compared to [67] and [68], the relay-aided MS361 DSD scheme of [69] further mitigates the system's complexity 362 and exhibits an increased flexibility in terms of adapting to

\footnotetext{
${ }^{3}$ Hence, in the perspective of achievable BER performance, we categorize DF-DD into sub-optimum MSDD in Fig. 3.
}

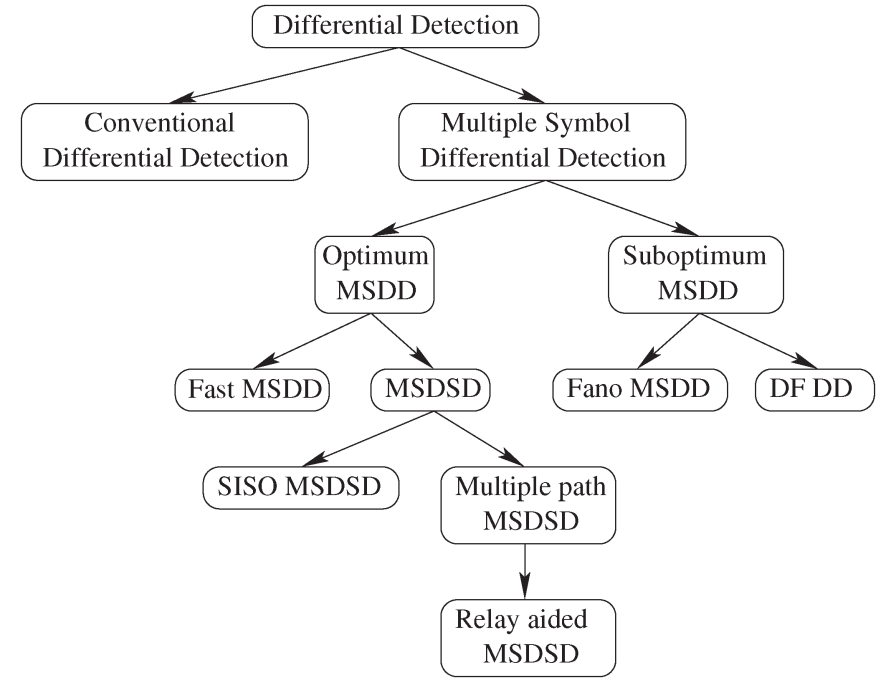

Fig. 3. Evolution of the multiple-symbol differential detectors: CDD [74], MSDD [53], [73], Fast-MSDD [54], DF-DD [57], [60], Fano-MSDD [63], MSDSD [62], SISO-MSDSD [66], Multiple-path MSDSD [67], Relay-aided MSDSD [69], [70].

multi-user, multi-relay scenarios. As a further advance, the soft- 363 decision based version of the relay-aided MSDSD was extended 364 in [70], to create the relay-aided soft-input soft-output MSDSD 365 (relay-aided SISO-MSDSD).

We summarize the above-mentioned history of multiple- 367 symbol differential detection techniques in Table IV. Similar 368 to [71], Fig. 3 is provided for visualizing the evolution of the 369 noncoherent differential detection techniques.

Based on the above brief review of NC detection techniques, 371 in view of their benefits in obviating the need for channel es- 372 timation and in overcoming the problem that we cannot expect 373 the mobile relays to estimate the source-to-relay channels, we 374 advocate the family of NC detection techniques, especially the 375 MSDSD algorithms of [62] and [66] as well as their relay- 376 aided MSDSD variants [69], [70] as the detection strategy to 377 be used for successive relaying aided cooperative networking. 378 Hence, in the rest of this treatise, we focus our discussions on 379 advanced non-coherent successive relaying (NC-SR).

\section{A. Outline of the Paper}

As addressed early in Section I, to avoid the potential 382 correlation of the antenna elements routinely encountered in 383 conventional MIMO systems, while retaining the spectral ef- 384 ficiency of the multi-antenna systems, the successive relaying 385 aided cooperative network was advocated. Then, for the sake of 386 circumventing the potentially excessive-complexity and hence 387 power-hungry channel estimation, whilst still exhibiting a com- 388 petitive BER performance, the family of noncoherent differen- 389 tial multiple-symbol joint detection algorithms was investigated 390 in the context of advanced NC-SR regimes. Hence, our goal 391 with this review is to stimulate further research and to inspire 392 additional novel contributions on spectrally-efficient cooper- 393 ative systems dispensing with channel-estimation.

In our forthcoming discourse, in general, we will portray the 395 historic development of the NC-SR regime from its original 396 
TABLE IV

Contributions to Multiple-Symbol DifFerential Detection

\begin{tabular}{|c|c|c|}
\hline Year & Authors & Contributions \\
\hline 1968 & Van Trees [72] & Contributed the critical theoretical foundation of the MSDD algorithm. \\
\hline 1990 & Divsalar et al. [53], [73] & $\begin{array}{l}\text { Heralded the MSDD algorithm by using a multiple-symbol observation interval } \\
\text { during the differential detection process and analysed its BER performance in } \\
\text { AWGN channels. }\end{array}$ \\
\hline 1992 & Ho and Fung [59] & $\begin{array}{l}\text { Redesigned the decision metric of the MSDD algorithm [53] by introducing the } \\
\text { channel's correlation matrix. As a result, the application of the MSDD algorithm } \\
\text { was extended to correlated Rayleigh fading channels. The associated BER } \\
\text { performance was analysed. }\end{array}$ \\
\hline 1994 & Mackenthun [54] & $\begin{array}{l}\text { Developed a fast algorithm for MSDD [53], which imposes a complexity order } \\
\text { of only } N \log _{2}(N) \text { per } N \text { symbols in AWGN channels. }\end{array}$ \\
\hline 1999 & Schober et al. [60] & $\begin{array}{l}\text { Extended the DF-DD algorithm to Rayleigh fading channels, where the } \\
\text { per-symbol complexity of the MSDD algorithm grows linearly with the } \\
\text { observation window size. Consequently, a sub-optimum trade-off between the } \\
\text { performance attained and the complexity imposed was struck. }\end{array}$ \\
\hline \multirow{2}{*}{2005} & Lampe et al. [62] & $\begin{array}{l}\text { Further developed the MSDD algorithm to the contemporary state-of-the-art } \\
\text { noncoherent detection regime - MSDSD. }\end{array}$ \\
\hline & Pun and Ho [63] & $\begin{array}{l}\text { Proposed the Fano-MSDD algorithm as an alternative technique of MSDSD for } \\
\text { low-SNR scenarios. }\end{array}$ \\
\hline 2006 & Pauli et al. [66] & Devised the energy-efficient, soft-decision-aided SISO-MSDSD regime. \\
\hline 2009 & Wang and Hanzo [67] & $\begin{array}{l}\text { Applied the MSDSD algorithm to cooperative networks, and consequently } \\
\text { generated the multiple-path MSDSD. }\end{array}$ \\
\hline 2011 & Li et al. [69], [70] & $\begin{array}{l}\text { Created both the hard-decision and soft-decision based relay-aided MSDSD, } \\
\text { which was exclusively designed for handling multiple input information streams } \\
\text { at the DN of a cooperative network, while dispensing with CSI. }\end{array}$ \\
\hline
\end{tabular}

397 basic representative to its most sophisticated architecture, step 398 by step. In each step, we may introduce a number of basic con399 cepts and principles, portray the system's architecture, identify 400 the fundamental challenges encountered, detail the associated 401 solutions, consider the realistic transceiver design principles, as 402 well as derive a variety of theoretical performance bounds.

403 In more detail, we will offer further insights into the advan404 tages of the successive relaying (SR) regimes in Section II-A, 405 where the SR regime and other relaying regimes, as well as 406 among different SR regimes are compared. Then, the trade407 off between the BER performance gain attained and the com408 plexity imposed by the MSDSD algorithm is characterised in 409 Section II-C, while the mathematical derivation leading from 410 the MSDSD algorithm of [62] to the HDB relay-aided MSDSD 411 and further to the SDB relay-aided MSDSD is provided in 412 Section II-D and E. The design principles of these algorithms 413 are also highlighted. These tutorial components focusing on 414 both the SR regime and on the relay-aided MSDSD algorithm 415 can be regarded as preliminaries for the ensuing discussions of 416 our original contributions.

417 Consecutively, two prototypes are introduced herein, namely 418 the successive AF relaying aided direct-sequence code-decision 419 multiple-access (DS-CDMA) uplink of Section III and the 420 successive DF relaying aided DS-CDMA uplink of Section IV. 421 In Section III, we move forward from the "single-user" scenario 422 to the "multi-user" scenario. In Section IV, we equip the system 423 with a powerful error correction capability.

424 Finally, we conclude in Section V. Some potential future 425 research topics in the area of NC-SR are also highlighted in 426 Section V. For the sake of facilitating the readers, the glossary 427 is shown in Table XIV. Hence, the overall structure of this 428 treatise may be summarized as in Fig. 4, in which DCMC is an 429 abbreviation for discrete-input continuous-output memoryless 430 channel.

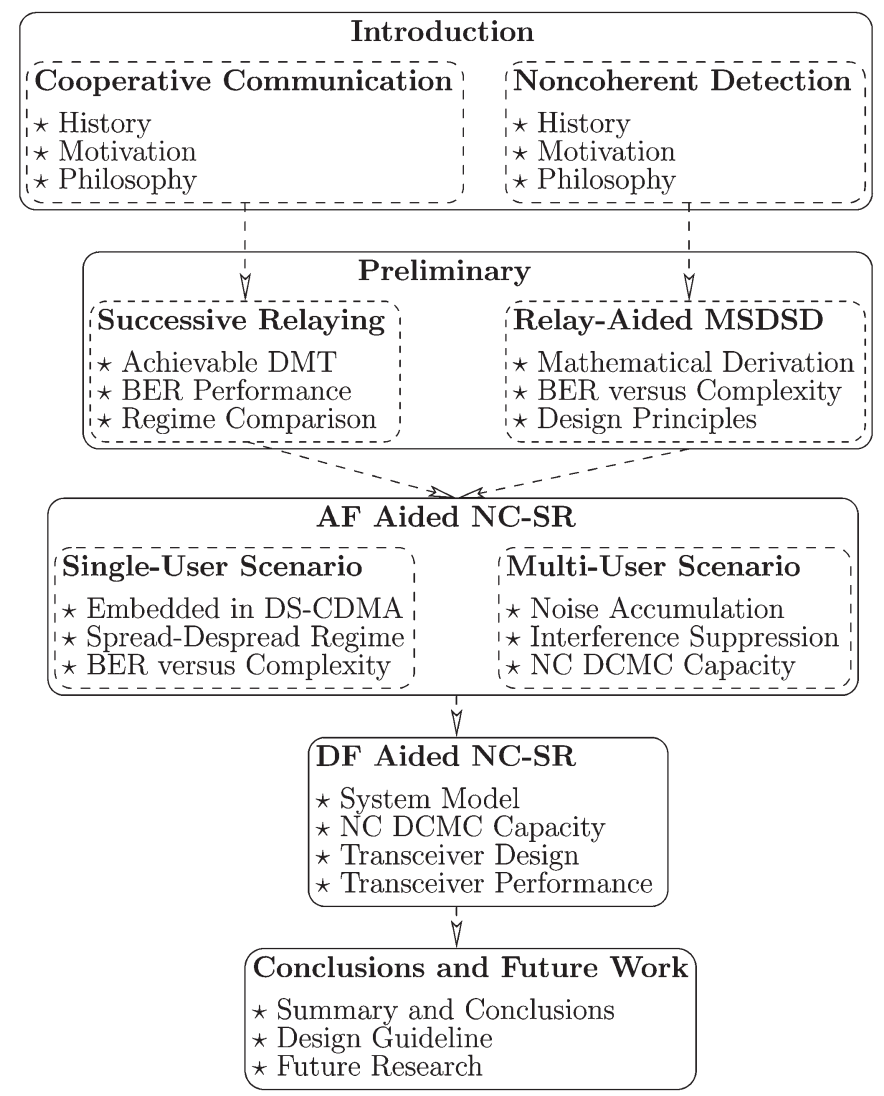

Fig. 4. Outline of the paper.

II. Preliminary: AdVAntages of SR And The 431 EMERGENCE OF RELAY-AIDED MSDSD

A. Advantages of Successive Relaying

The transmission schedule of the successive relaying proto- 434 col was illustrated in Fig. 2. However, for clarifying it further by 435 


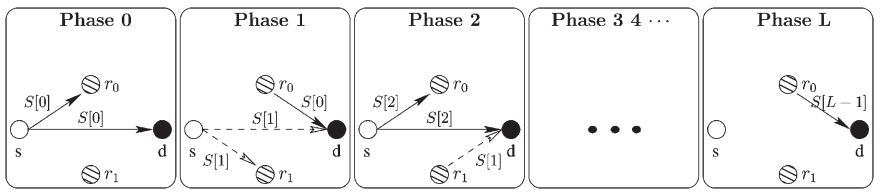

Fig. 5. Simplified transmission schedule of the successive relaying protocol, where the IRI is omitted.

436 focusing only on the desired signal components whilst ignoring 437 the effects of the IRI, the simplified transmission arrangement 438 of the successive relaying protocol is displayed in Fig. 5. 439 Correspondingly, the specific actions involved in every phase 449 shown in Fig. 5 are described as follows

- Phase 0: The SN $s$ broadcasts the message $S[0]$; the RN $r_{0}$ listens to the $\mathrm{SN} s$ and receives the message $S[0]$; the $\mathrm{RN} r_{1}$ remains silent; the $\mathrm{DN} d$ receives the message $S[0]$.

463 As mentioned earlier, Fan et al. [46] analysed the DMT ${ }^{4}$ of 464 DF based successive relaying, while assuming perfect source465 to-relay links. The algebraic relationship between the diversity 466 order and the associated multiplexing gain was also formulated 467 by them in [46, (33)]. Based on [46, (33)], the DMT struck 468 by successive DF relaying relying on $L$ processing phases is 469 depicted in Fig. 6. The DMTs achieved by direct transmission, 470 conventional DF relaying, as well as conventional AF relaying 471 are also shown in Fig. 6 as the benchmarks, which are directly 472 abstracted from [11, Fig. 6]. As argued before, successive DF 473 relaying is capable of achieving a significantly improved DMT 474 compared to conventional DF or AF relaying. This specific 475 benefit of successive relaying is evidenced again in Fig. 6.

476 For the sake of extending our horizon further as well as 477 of further manifesting the advantage of SR, we would like 478 to compare several typical relaying regimes herein, which 479 include full-duplex relaying (FDR), SR, two HD relays aided

\footnotetext{
${ }^{4}$ In general, a MIMO system (including cooperative systems) can provide two types of gains: diversity gain and multiplexing gain. However, maximizing one type of gain may not necessarily maximize the other. To compare the performance between diversity-oriented and multiplexing-oriented schemes, Zheng and Tse defined the concept of DMT $D(r)$ in [5], which represents the diversity order $D$ as a function of the multiplexing gain $r$. The DMT is essentially the trade-off between the error probability and the data rate of a system.
}

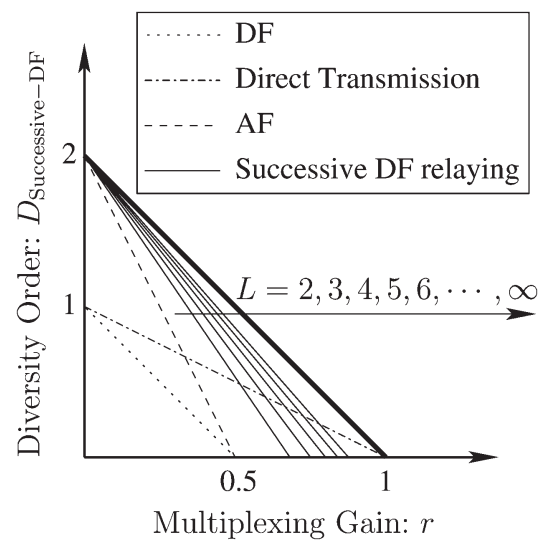

Fig. 6. Diversity order versus multiplexing gain $r$ for the successive DF relaying protocol, where the number of processing phases $L$ is increased from $L=2$ to $\infty$. The relevant curves are attained according to [46, (33)]. Then, according to the transmission process depicted in Fig. 5, intuitively, the normalized throughput of successive DF relaying (or its multiplexing gain) is limited to the region of $r \in\left[0, \frac{L}{L+1}\right]$. The DMT of both the conventional DF relaying and of the AF relaying as portrayed in [11, Figure 6] are also included here as a pair of benchmarks.

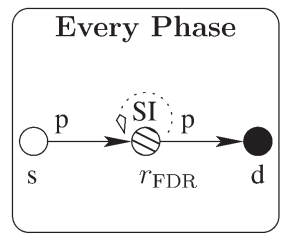

(a)

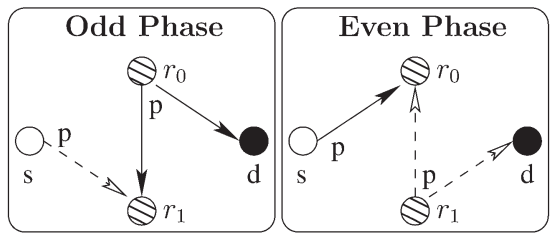

(b)

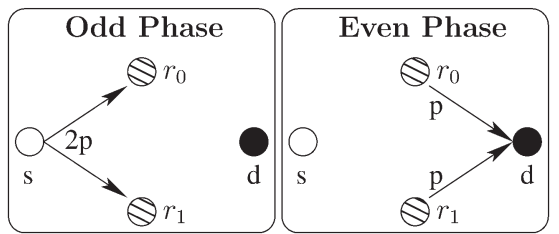

(c)

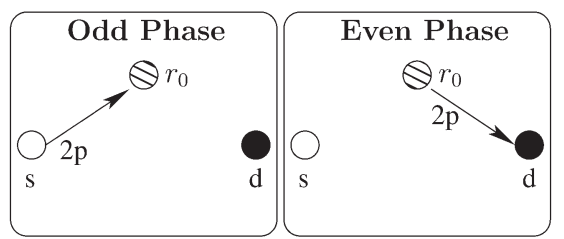

(d)

Fig. 7. Transmission framework of a range of typical relaying regimes. The detailed interpretation of each regime can be found in Table V. (a) Full duplex relaying. (b) Successive relaying. (c) Two HD relays aided CR. (d) Single HD relay aided $\mathrm{CR}$.

conventional relaying (CR), as well as a single HD relay aided 480 CR. Their transmission frameworks are compactly illustrated 481 in Fig. 7 and the associated interpretations can be found in 482 Table V. For the sake of a fair comparison, the transmit power of 483 all the competitive relaying regimes is fixed to $2 P$. For example, 484 
TABLE V

DETAILED INTERPRETATIONS OF THE RELAYING REGIMES INVOLVED IN Fig. 7

\begin{tabular}{|l|l|}
\hline full-duplex relaying (FDR) & $\begin{array}{l}\text { A full-duplex relay node is employed, which is capable of transmitting and } \\
\text { receiving signals simultaneously. Hence the SN-to-RN and the RN-to-DN } \\
\text { transmissions can be completed in a single phase. }\end{array}$ \\
\hline \multirow{3}{*}{ successive relaying (SR) } & $\begin{array}{l}\text { Relying on a large number of processing phase of } L \text {, we can only utilize the "Phase } \\
1 \text { " and "Phase 2" in Figure 2 to summarize the entire transmission arrangement of } \\
\text { SR. If we further ignore the direct SN-to-DN link owing to its large distance, we } \\
\text { obtain "(b) successive relaying" in Figure 7. }\end{array}$ \\
\hline \multirow{2}{*}{ two HD relays aided CR } & $\begin{array}{l}\text { During the broadcast phase, the SN } s \text { broadcast its information, while two RNs } r_{0}, \\
r_{1} \text { listen to the SN. During the cooperative phase, two RNs } r_{0}, r_{1} \text { simultaneously } \\
\text { forward the SN's information heard in the last phase to the DN. }\end{array}$ \\
\hline \multirow{2}{*}{ single HD relay aided CR } & $\begin{array}{l}\text { It is the fundamental relaying prototype and has a similar transmission arrangement } \\
\text { to that of "(c) two HD relays aided CR". By contrast, instead of exploiting multiple } \\
\text { RNs, it only employs a single HD RN. }\end{array}$ \\
\hline
\end{tabular}

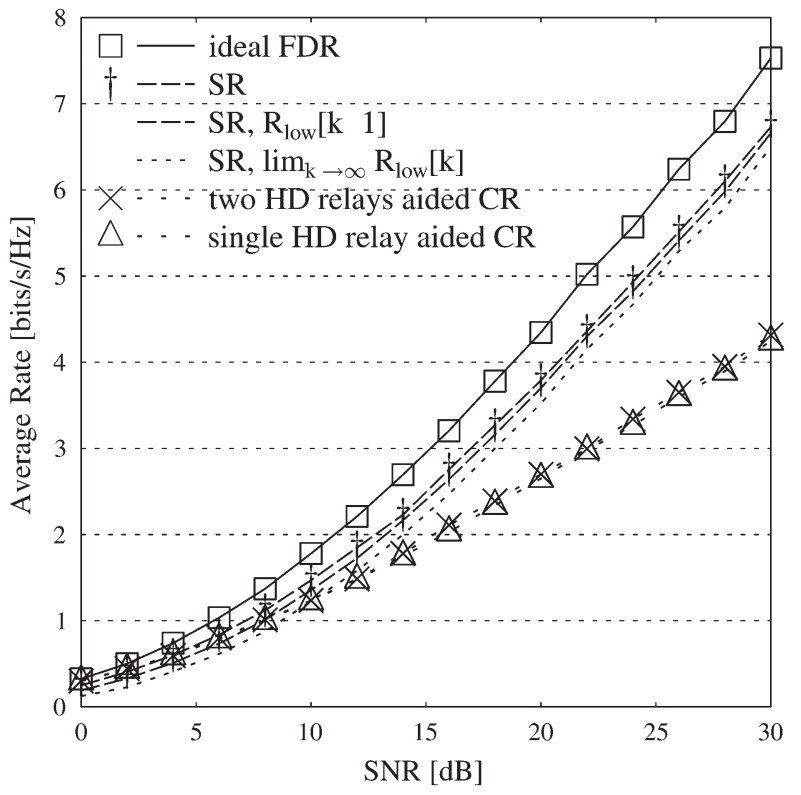

Fig. 8. Performance of several relaying schemes in terms of average achievable error-free data rate versus SNR. All the competitive relaying regimes employ AF relaying protocol. Particularly, it is assumed that the SI incurred in the "full-duplex relaying" of Fig. 7(a) has been perfectly cancelled, which results in the curve marked "ideal FDR" in this figure. Then, the strength of IRI incurred in SR is assumed to be the half of the desired signals. These simulation results are based on [34] and they can also be found in [34, Fig. 6].

485 since there are always two transmitters in every phase of "(b) 486 successive relaying", both the $\mathrm{SN} s$ and the RN $r_{i}, i \in 0,1$ 487 transmit their signals at the power of $P$. By contrast, in each 488 phase of "(d) single HD relay aided CR", there is only a single 489 transmitter. Hence, either the SN $s$ or the RN $r_{0}$ transmits 490 signals at the power of $2 P$. Furthermore, it is assumed that 491 the direct Source-to-Destination link is negligible owing to the 492 associated large propagation distance.

493 Consequently, the average achievable error-free data rate 494 versus SNR performance of each relaying regime of Fig. 7 is 495 shown in Fig. 8, where all the cooperative regimes employ 496 the AF relaying protocol. In [34], three different regimes were 497 considered, namely the SR regime employing adaptive rate 498 allocation, the SR regime transmitting exactly at the ergodic 499 capacity of time slot $[k+1]$ as the fixed transmit rate of its $500 \mathrm{SN}$, as well as the SR regime transmitting at the ergodic 501 capacity of time slot $k \rightarrow \infty$ as the fixed transmit rate of its
SN. They are denoted by "SR", "SR, $R_{\text {low }}[k+1]$ ", and "SR, 502 $\lim _{k \rightarrow \infty} R_{\text {low }}[k]$ " in Fig. 8, respectively. Observe in Fig. 8 that 503 replacing adaptive rate allocation by the fixed rate allocation 504 scheme will sightly degrade the performance of SR. Neverthe- 505 less, the performance of these SR regimes is still close to that 506 of the "ideal FDR". Furthermore, they significantly exceed the 507 performance of the "single HD relay aided CR" or of the "two 508 HD relays aided CR" beyond SNR $=10 \mathrm{~dB}$. More particularly, 509 observe in Fig. 7 that both the "(b) successive relaying" and 510 the "(c) two HD relays aided CR" require an identical number 511 of RNs. However, the SR regime is capable of substantially 512 outperforming its conventional relaying based counterpart. This 513 fact underlines the advantage of the SR regime.

514

Furthermore, depending on the specific type of protocol 515 employed at the RN, the SR regime illustrated in Fig. 7 can be 516 categorised into AF aided SR and DF aided SR. The AF aided 517 SR regime has been investigated in Fig. 8. Then, according to 518 whether the RNs do or do not invoke IRI cancellation, the DF 519 aided SR can be further categorised into two types, namely the 520 "DF aided SR, where the RNs dispense with IRI canc." and the 521 "DF aided SR, where the RNs employ IRI canc.". Hence, it is 522 worth providing further insights into SR by comparing these 523 different SR regimes. As revealed in [34], the performance of 524 these different SR regimes is predetermined by their ability to 525 mitigate the IRI effects. Hence it is insightful to compare their 526 error-free data rates versus the IRI power, which are shown 527 in Fig. 9, where the "ideal DF aided FDR" system is similar 528 to the "ideal FDR" system shown in Fig. 8, but with its AF 529 protocol employed at the FD relay replaced by the DF protocol. 530 Then, the "AF aided SR, DN employs IRI canc." system of 531 Fig. 9 is the same as the system, namely "SR" in Fig. 8. 532 Furthermore, the "single HD-DF relay aided CR" system of 533 Fig. 9 is obtained upon replacing the AF protocol employed 534 by the "single HD relay aided CR" system in Fig. 8, by the DF 535 protocol. Furthermore, the IRI to signal power ratio (ISR) is 536 used as the horizontal axis in Fig. 9.

Observe in Fig. 9 that for ISR $\geq 10 \mathrm{~dB}$, the RNs of the SR 538 system should perform the IRI cancellation before detecting the 539 messages transmitted by the SN. Specifically, it is beneficial 540 to invoke the regime, namely "DF aided SR, RNs employ IRI 541 canc.". By contrast, if the IRI is sufficiently weak, e.g. when 542 we have ISR $\leq-15 \mathrm{~dB}$, it is not necessary to invoke IRI 543 cancellation at the RNs of the SR system. Instead, it is better 544 to directly decode the signals transmitted by the SN at the RNs 545 


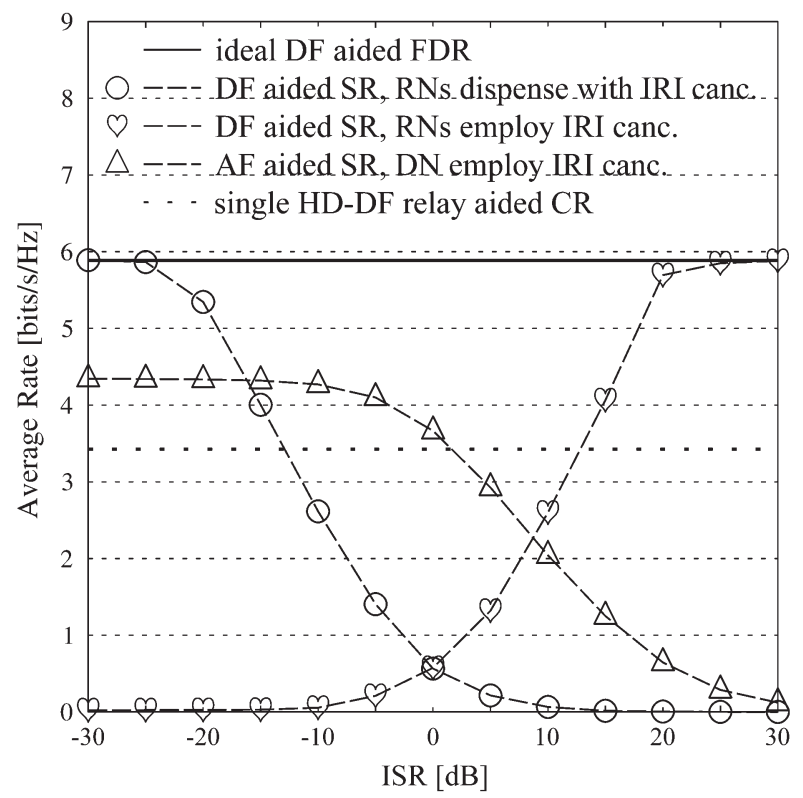

Fig. 9. Comparison among different SR regimes in terms of their average achievable error-free data rates versus ISR. The signal to noise power ratio of the desired signal is fixed to $\mathrm{SNR}=20 \mathrm{~dB}$. These simulation results are based on [34] and they can also be found in [34, Fig. 9].

546 of the SR system, while considering the IRI to be an additional 547 noise component. Hence, in this case, we may advocate the 548 "DF aided SR, RNs dispense with IRI canc." regime. In the 549 case of incurring weak or moderate IRI, for example, when we 550 have ISR $\in(-15,10) \mathrm{dB}$, we may opt for the AF aided SR 551 regime. Since our DS-CDMA based interference suppression 552 technique, which will be introduced in Section III-A and C2 553 is capable of sufficiently suppressing the IRI. Generally, in 554 Sections III and IV, we operate the SR systems in weak IRI 555 scenarios. Hence, we may focus our attention on AF aided SR, 556 as well as on DF aided SR, where the RNs directly decode the 557 signals transmitted by the $\mathrm{SN}$, while regarding the IRI as an 558 additional noise component.

\section{B. Precondition of Embedding Successive Relaying 560 Into DS-CDMA System}

561 As summarized in Table III, SR imposes both co-channel 562 interference and inter-relay interference at the DN. These SR 563 induced interferences may significantly degrade the benefits of 564 the SR [34], [46].

565 Against this background, we invoked the DS-CDMA tech566 nique in [75] and designed the successive relaying aided coop567 erative DS-CDMA uplink. In this transmission framework, the 568 successive relaying aided network (SRAN) is embedded in the 569 DS-CDMA uplink for improving the communication quality of 570 a cell-edge user. Hence, the DS-CDMA technique is used for 571 mitigating the successive relaying induced interference between 572 the transmitted signals of the source and relay.

573 In more detail, for the sake of a fair comparison to the 574 conventional two-phase cooperative system [11], we have to 575 ensure that no extra channel resources are required by the 576 advocated successive relaying aided cooperative DS-CDMA
TABLE VI

SYSTEM PARAMETERS EMPLOYED IN FIGS. 11 AND 12

\begin{tabular}{|l|r|}
\hline Scenario & Depicted in Figure 10 \\
\hline Channel Model & Narrow-Band Flat-Fading Channel \\
\hline Normalized Doppler Frequency & $f_{d}=0.03$ \\
\hline Modulation & 4-PSK for Coherent Detection \\
& 4-DPSK for Differential Detection \\
\hline \multirow{2}{*}{ Detector } & Coherent Detector \\
& Coherent Differential Detector \\
& NC Differential Detector \\
\hline Window Size of MSDSD & SL MSDSD (or MSDD) \\
\hline
\end{tabular}

uplink. Practically, governed by a certain target BER value, 577 there may be a strict limit on the number of active users that 578 can be supported in a DS-CDMA system, which we denote by 579

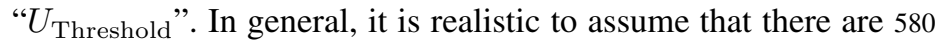
some idle users in a DS-CDMA cellular system. Hence, the 581 preconditions of employing the SRAN in support of a cell-edge 582 user in the DS-CDMA system may be as follows: 583

1. We find a sufficient number of idle users between the SN 585 and DN, willing to support the cell-edge user. 586

2. The sum of the number of active users and relays, namely 587 $U_{\mathrm{ACT}}+U_{\mathrm{RN}}$ is still lower than $U_{\text {Threshold }}$.

In other words, the relays employed in the SRAN are the idle 589 users of the DS-CDMA cellular system. Moreover, since we 590 assume that $U_{\mathrm{ACT}}+U_{\mathrm{RN}}<U_{\text {Threshold }}$, the system does not 591 run out of spreading sequences. However, appointing idle users 592 to serve as the relays increases the total number of active 593 entities in the DS-CDMA cellular system, hence increasing the 594 interference. Therefore, it may be concluded that the proposed 595 successive relaying aided cooperative DS-CDMA uplink es- 596 sentially converts the typical 50\% half-duplex relaying-induced 597 throughput loss to a potential user-load reduction of the CDMA 598 system.

\section{MSDSD: Improving the Trade-Off Between the Power Gain 600 and Decoding Complexity

As stated early in Section I, CDD will exhibit a high error- 602 floor in fading scenarios. This is demonstrated in Fig. 11 by the 603 dashed curve marked by the heart legends, where a flat Rayleigh 604 fading channel having a normalized Doppler frequency of $f_{d}=605$ 0.03 was considered. As summarized in Table IV, for the sake 606 of accommodating fading channels, Ho et al. [59] as well as 607 Divsalar et al. [58] introduced the channel correlation matrix 608 into the initial MSDD algorithm proposed by Divsalar et al. in 609 [53]. By exploiting both the increased time-diversity gain of the 610 extended observation window size and the statistical knowledge 611 of the channel, the BER performance of the improved MSDD 612 algorithm [58], [59] is capable of approaching the lower bound 613 of differential detection, namely that achieved by coherent dif- 614 ferential detection. The beneficial effect of increasing the obser- 615 vation window size is demonstrated by the solid lines in Fig. 11. 616

However, when employing $M_{c}$-ary differential phase-shift 617 keying $\left(M_{c}\right.$-DPSK) and an observation window size of $N, 618$ to make a decision concerning the differential symbol se- 619 quence $\mathbf{S}$ transmitted during an observation window, the MSDD 620 


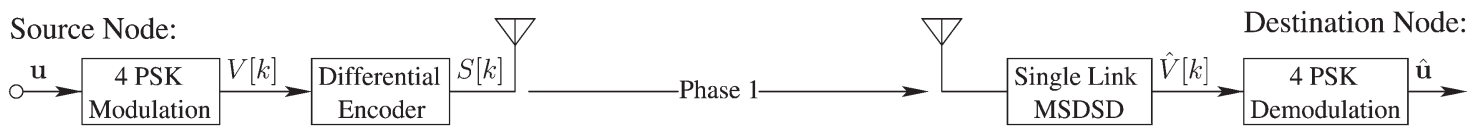

Fig. 10. Schematic of single-link MSDSD.

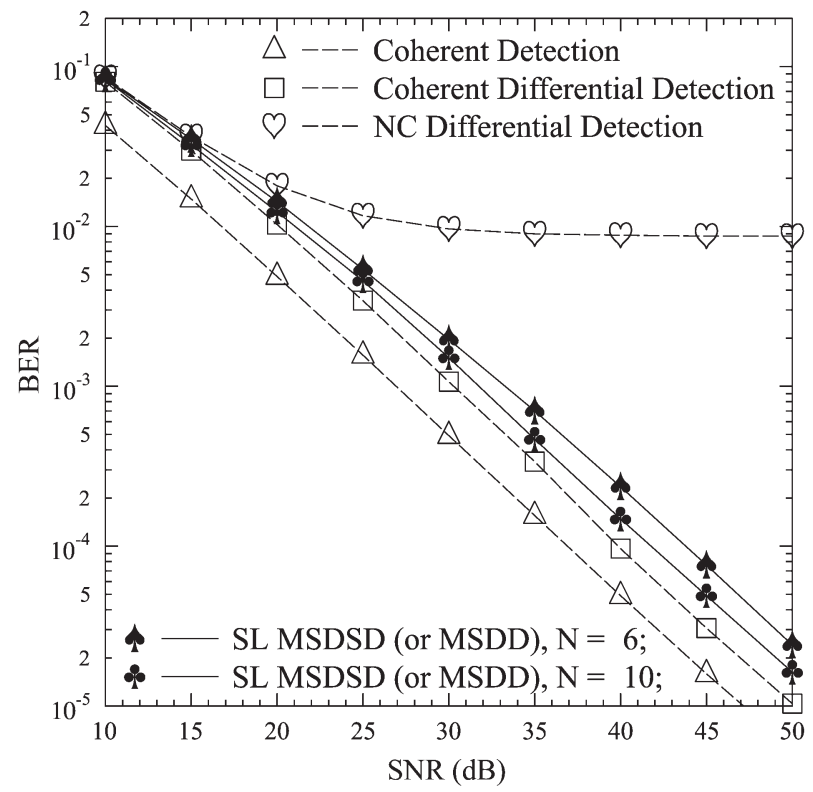

Fig. 11. BER performance of the single-link (SL) direct-transmission (DT) based MSDSD (or MSDD) when encountering a flat Rayleigh fading channel having a normalized Doppler frequency of $f_{d}=0.03$ and employing 4-DPSK modulation. The associated BER performance of coherent detection, coherent differential detection, and conventional differential detection are also portrayed. The results for SL MSDSD (or MSDD) are based on the schematic of Fig. 10.

621 algorithm has to test all of its $M_{c}^{N-1}$ legitimate combinations. ${ }^{5}$ 622 In more detail, each Frobenius norm, ${ }^{6}$ which is determined 623 by both a specific legitimate combination of $\mathbf{S}$ and the actual 624 received signal sequence is calculated. Then, the particular 625 legitimate combination of $\mathbf{S}$, which results in the minimum 626 Frobenius norm is deemed to be the optimum decision. We may 627 refer to Fig. 16, which visualizes the search space of the MS628 DSD algorithm [62], as will be discussed later in Section II-D 629 for the sake of capturing the philosophy of this detection pro630 cess. Hence, the Frobenius norm calculation (FNC) constitutes 631 the major contribution of the system's decoding complexity. 632 Accordingly, the average number of FNCs required for decod633 ing a single differential symbol sequence $\mathbf{S}$ can be employed 634 as a basic metric for evaluating the decoding complexity of 635 the multiple symbol joint detection based algorithms, such as 636 MSDD and the MSDSD. ${ }^{7}$

637 In this spirit, the MSDD algorithm imposes a decoding com638 plexity of $M_{c}^{N-1}$ FNCs per observation window. By contrast, 639 the CDD algorithm only requires $M_{c}$ FNCs per observation 640 window. Hence, the power gain of the MSDD algorithm with

\footnotetext{
${ }^{5}$ As a reference symbol, the last differential symbol of an observation interval is always fixed, which therefore does not require any detection.

${ }^{6}$ Its precise definition is given in (4) of Section II-D.

${ }^{7}$ The complexity imposed by the sorting operation during the differential decoding process of MSDSD may be ignored for the sake of simplifying the complexity comparison among CDD, MSDD and MSDSD.
}

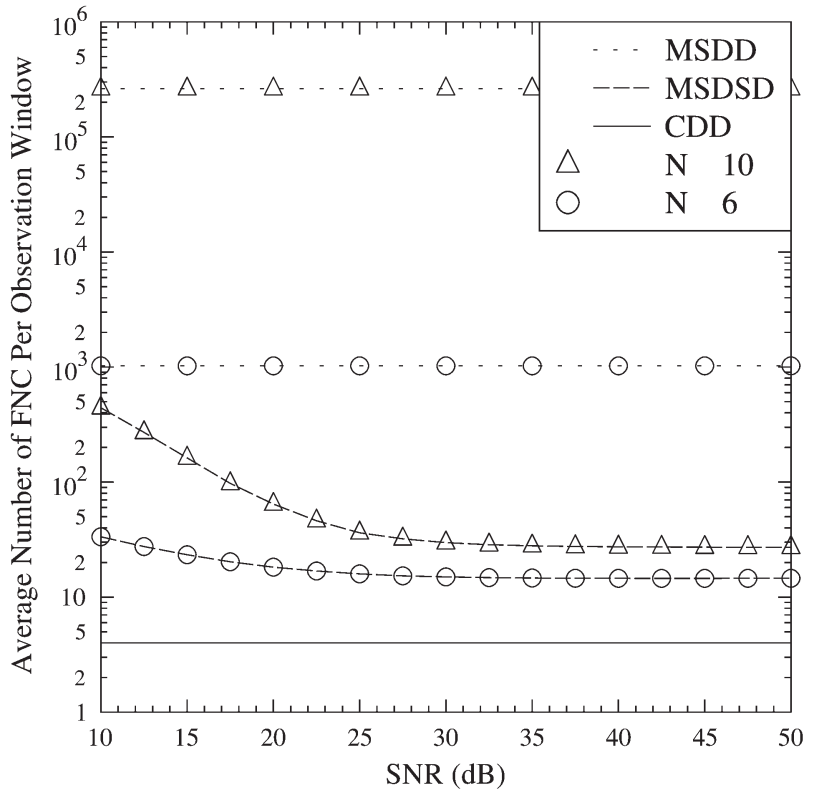

Fig. 12. The decoding complexities associated with the CDD, MSDSD and MSDD schemes employed in Fig. 11 are compared here, where the average number of FNCs required for decoding a single observation interval is employed as the complexity metric. The results are based on the schematic of Fig. 10 as well as on the parameters of Table VI.

respect to the CDD algorithm as shown in Fig. 11 is obtained at 641 the cost of imposing a significantly higher decoding complexity. 642 Therefore, an improvement of the trade-off between the power 643 gain obtained and the associated decoding complexity was 644 desired. With this objective, Lampe et al. [62] devised the pow- 645 erful MSDSD algorithm, which is capable of excluding some 646 legitimate combinations of $\mathbf{S}$ from the search space without 647 going all the way to completing their associated FNCs. As a 648 benefit, the brute-force maximum-likelihood search involved in 649 the MSDD algorithm is obviated without sacrificing the attain- 650 able BER performance. This decoding complexity reduction 651 facilitated by the MSDSD algorithm is demonstrated in Fig. 12. 652 Based on Figs. 11 and 12, the MSDSD algorithm significantly 653 reduces the decoding complexity of the MSDD algorithm, 654 while retaining its BER performance. Hence the compromise 655 between the power gain obtained and the associated decoding 656 complexity of the NC detection techniques is improved.

To further improve the trade-off between the power gain 658 achieved and the decoding complexity of NC detection, the 659 single-path MSDSD algorithm of [62] was further developed 660 into a novel multiple-path form in [67] and [68], where the 661 original MSDSD algorithm was appropriately modified and 662 applied to cooperative systems. For example, the schematics of 663 the cooperative systems relying on the "twin-path" MSDSD and 664 on the "triple-path" MSDSD are portrayed in Figs. 13 and 14, 665 respectively. 


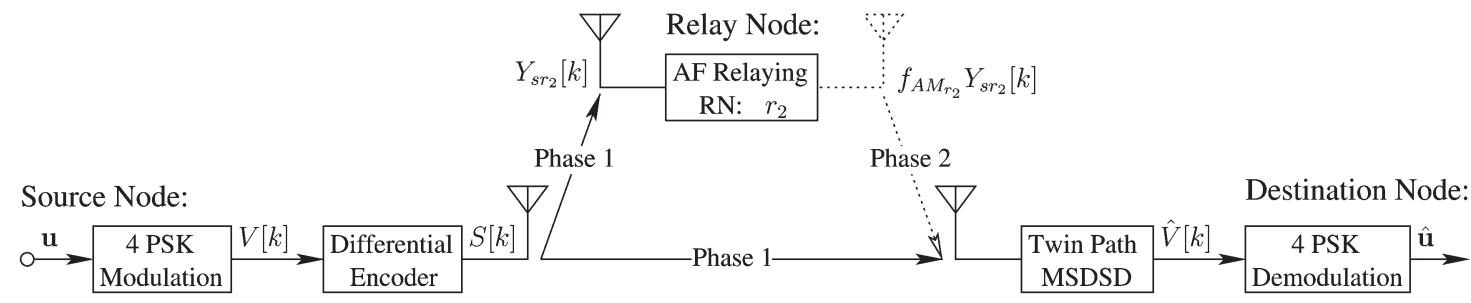

Fig. 13. Schematic of twin-path MSDSD based AF relaying.

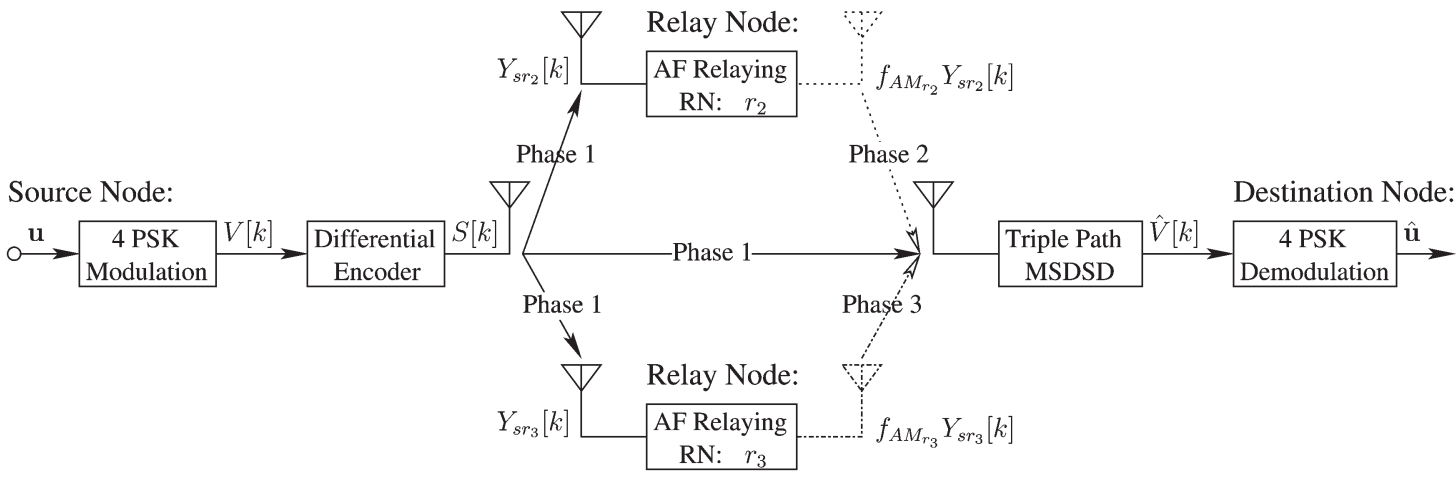

Fig. 14. Schematic of triple-path MSDSD based AF relaying.

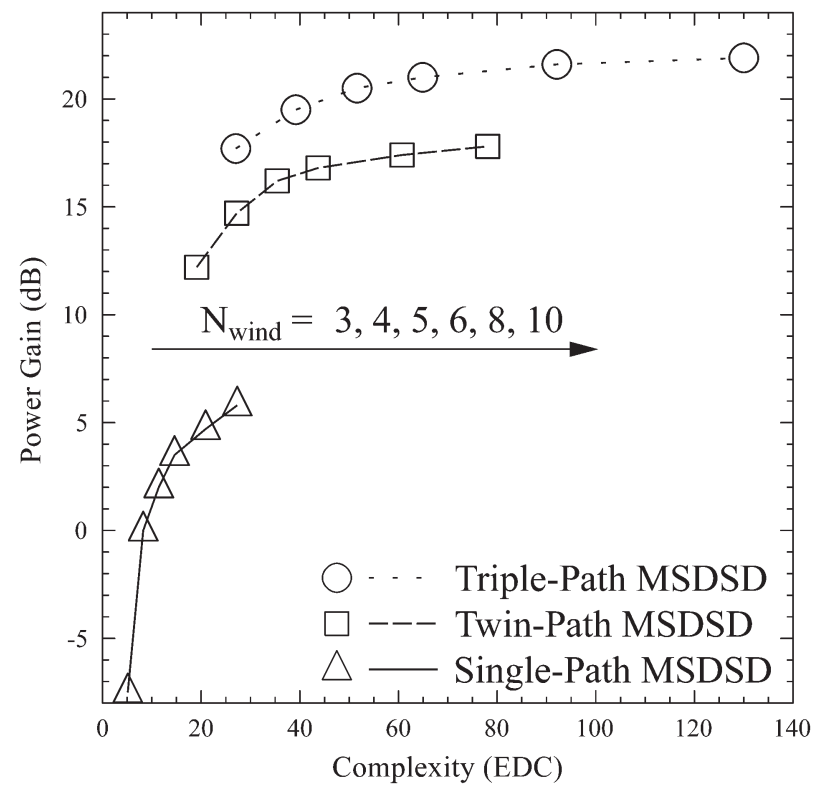

Fig. 15. Power gain versus complexity for single- and multiple-path MSDSD, where the power gain is expressed in terms of the SNR. Then, the SNR required by the single-path MSDSD algorithm having an observation window size of $N_{\text {wind }}=4$ for approaching the target BER of $10^{-4}$ when encountering a flat Rayleigh fading channel having $f_{d}=0.03$ is regarded as the power-gain benchmark. The results of the "single-path"system, the "twin-path" system and the "triple-path" system are based on the schematics of Figs. 10, 13, and 14, respectively, where all the parameters employed are summarized in Table VII.

667 The relevant improvement of the trade-off between the power 668 gain attained and the associated decoding complexity is re669 vealed in Fig. 15, where the SNR required by the single-path 670 MSDSD algorithm having observation window size of $N_{\text {wind }}=$ 6714 for approaching the target BER of $10^{-4}$ when encountering a 672 flat Rayleigh fading channel having $f_{d}=0.03$ is regarded as 673 our power-gain benchmark.
TABLE VII

SYSTEM PARAMETERS EMPLOYED IN FIG. 15

\begin{tabular}{|l|r|}
\hline Scenario & Depicted in Figures 10,13 and 14 \\
\hline Channel Model & Narrow-Band Flat-Fading Channel \\
\hline Normalized Doppler Frequency & $f_{d}=0.03$ \\
\hline Modulation & Differential QPSK \\
\hline & Single-Path MSDSD \\
Detector & Twin-Path MSDSD \\
& Triple-Path MSDSD \\
\hline Window Size of MSDSD & $N \in\{3,4,5,6,8,10\}$ \\
\hline
\end{tabular}

However, as commented in Section I, as well as shown 674 in Table IV, the multiple-path MSDSD [67], [68] remains 675 insufficiently flexible for application in cooperative networks, 676 especially when having a large number of propagation paths 677 in the cooperative network owing to employing multiple RNs. 678 Correspondingly, the relay-aided MSDSD of [69] and [70] was 679 devised, which can be readily applied to various cooperative 680 networks having an arbitrary number of RN induced propa- 681 gation paths. This is achieved without any BER performance 682 degradation with respect to multiple-path MSDSD [67], [68]. 683 The conception of both the HDB and the SDB relay-aided 684 MSDSD is briefly highlighted in Section II-D and E.

\section{HDB Relay-Aided MSDSD}

686

Let $\mathbf{V}$ denote an information symbol vector, which con- 687 sists of $(N-1) M_{c}$-ary PSK information symbols $V[k] \in 688$ $\left\{e^{j 2 \pi m / M_{c}} ; m=0,1, \cdots, M_{c}-1\right\}$. Then, $\mathbf{V}$ is differentially 689 encoded to a DPSK symbol vector $\mathbf{S}$ having a length of $N .690$ After $\mathbf{S}$ is transmitted from $u$ th entity (SN or RN) to the DN via 691 a flat Rayleigh fading channel having a CIR vector of $\mathbf{H}_{u}$, the 692 differential decoder in Fig. 10 will receive a signal vector $\mathbf{Z}_{u}, 693$ which is a distorted version of $\mathbf{S}$. Hence we have the following 694 relationships: $\mathbf{V}=[V[1], V[2], \ldots, V[N-1]]^{T}, \mathbf{S}=[S[1], S[2], 695$ 
$696 \ldots, S[N]]^{T}, \quad \mathbf{H}_{u}=\left[h_{u}[1], h_{u}[2], \ldots, h_{u}[N]\right]^{T}, \mathbf{Z}_{u}=\left[z_{u}[1]\right.$, $\left.697 z_{u}[2], \ldots, z_{u}[N]\right]^{T}, \mathbf{N}=[N[1], N[2], \ldots, N[N]]^{T}$ and

$$
\begin{aligned}
S[k+1] & =S[k] V[k], \\
\mathbf{Z}_{u} & =\mathbf{H}_{u} \circ \mathbf{S}+\mathbf{N},
\end{aligned}
$$

698 where $\circ$ denotes the Hadamard product, and we have $N[k] \sim$ $699 \mathcal{C N}\left(0, \sigma^{2}\right)$.

700 Obviously, there are $\left(M_{c}\right)^{N-1}$ legitimate combinations of 701 the information symbol vector $\mathbf{V}$, which may be denoted by 702 the set $\chi$. Hence the challenge encountered by the noncoherent 703 differential multiple-symbol joint decoder is that of finding the 704 actually transmitted information vector $\mathbf{V}$ out of the entire set $705 \chi$, when relying only on the received signal vector $\mathbf{Z}_{u}$. The 706 mathematical principle behind this problem relates to the so707 called "general Gaussian problem" [72].

708 Based on [72] and Divsalar's original MSDD algorithm [53], 709 Ho and Fund [59] rewrote the specific form of the conditional 710 probability density function (PDF) of the received signal vector $711 \mathbf{Z}_{u}$, conditioned on the transmitted DPSK symbol vector $\mathbf{S}$, 712 which may be formulated as

$$
\operatorname{Pr}\left(\mathbf{Z}_{u} \mid \mathbf{S}\right)=\frac{1}{(2 \pi)^{\frac{N}{2}} \| \Phi_{\mathbf{Z}_{u} \mathbf{Z}_{u} \|^{\frac{1}{2}}}} \exp \left(-\frac{1}{2} \mathbf{Z}_{u}{ }^{H} \boldsymbol{\Phi}_{\mathbf{Z}_{u} \mathbf{Z}_{u}}^{-1} \mathbf{Z}_{u}\right),
$$

713 where the correlation matrix is given by $\boldsymbol{\Phi}_{\mathbf{Z}_{u} \mathbf{Z}_{u}} \triangleq \operatorname{diag}\{\mathbf{S}\}$ $714\left\{\mathcal{E}\left\{\mathbf{H}_{u} \mathbf{H}_{u}{ }^{H}\right\}+\sigma_{n}^{2} \mathbf{I}_{N}\right\} \operatorname{diag}\left\{\mathbf{S}^{*}\right\}, \mathbf{I}_{N}$ is an $N$-dimensional 715 identity matrix and $\sigma_{n}^{2}=N_{0}$ is the noise variance.

716 Accordingly, the previously mentioned challenge encoun717 tered by the noncoherent differential multiple-symbol joint 718 decoder can be tackled by selecting the optimum DPSK symbol 719 vector $\hat{\mathbf{S}}$ out of all the $\left(M_{c}\right)^{N-1}$ legitimate candidate vectors, 720 which maximizes the conditional PDF presented in (2). Then, 721 the benefit of presenting the conditional $\operatorname{PDF} \operatorname{Pr}\left(\mathbf{Z}_{u} \mid \mathbf{S}\right)$ in 722 the form of (2) is that when the amplitude of any legitimate 723 DPSK symbol $S[k]$ is fixed to unity, the determinant $\left\|\boldsymbol{\Phi}_{\mathbf{Z}_{u} \mathbf{Z}_{u}}\right\|$ 724 in (2) becomes independent of the DPSK symbol vector $\mathbf{S}$. 725 Hence, when comparing the values of the conditional PDF 726 corresponding to different DPSK symbol vectors, we only have 727 to concentrate our attention on the $\left(\mathbf{Z}_{u}{ }^{H} \boldsymbol{\Phi}_{\mathbf{Z}}^{-1} \mathbf{Z}_{u} \mathbf{Z}_{u}\right)$ part on the 728 right hand side of (2). Since the conditional $P D F \operatorname{Pr}\left(\mathbf{Z}_{u} \mid \mathbf{S}\right)$ 729 in (2) is a monotonically decreasing function of the metric $730\left(\mathbf{Z}_{u}{ }^{H} \boldsymbol{\Phi}_{\mathbf{Z}_{u} \mathbf{Z}_{u}}^{-1} \mathbf{Z}_{u}\right)$, maximising $\operatorname{Pr}\left(\mathbf{Z}_{u} \mid \mathbf{S}\right)$ over the entire set of $731 \mathbf{S}$ is equivalent to minimising $\left(\mathbf{Z}_{u}{ }^{H} \boldsymbol{\Phi}_{\mathbf{Z}_{u} \mathbf{Z}_{u}}^{-1} \mathbf{Z}_{u}\right)$ over the entire 732 set of $\mathbf{S}$. Hence, the decision rule is simplified to

$$
\hat{\mathbf{S}}=\underset{\mathbf{S} \in \chi}{\arg \min }\left\{\mathbf{Z}_{u}{ }^{H} \boldsymbol{\Phi}_{\mathbf{Z}_{u} \mathbf{Z}_{u}}^{-1} \mathbf{Z}_{u}\right\} .
$$

733 However, according to the decision rule (3), the minimum 734 decision metric $\left(\mathbf{Z}_{u}^{H} \boldsymbol{\Phi}_{\mathbf{Z}_{u}}^{-1} \mathbf{Z}_{u} \mathbf{Z}_{u}\right)$ can only be determined, if 735 all the $\left(M_{c}\right)^{N-1}$ legitimate combinations of the DPSK symbol 736 vector $\mathbf{S}$ are substituted into (3) and evaluated. To mitigate this 737 brute-force search problem incurred by the MSDD algorithms 738 of [53] and [59], Lampe et al. [62] devised the state-of-the739 art NC detection philosophy termed the MSDSD. In [62], 740 the refined decision rule proposed by Ho and Fund [59] and formulated in (3) may be expressed after a range of algebraic 741 manipulations as

$$
\hat{\mathbf{S}}=\underset{\mathbf{S} \in \chi}{\arg \min }\left\{\left\|\mathbf{U}^{u} \mathbf{S}\right\|^{2}\right\},
$$

where, the notation $\|\cdot\|$ denotes the Frobenius norm, $\mathbf{U}^{u} \triangleq 743$ $\left(\mathbf{F} \operatorname{diag}\left\{\mathbf{Z}_{u}\right\}\right)^{*}, \mathbf{U}^{u} \in \mathbb{C}^{N \times N}$, and $\mathbf{F}$ is a triangular matrix, 744 defined via $\mathbf{C}^{-1}=\mathbf{F}^{H} \mathbf{F}$, where $\mathbf{C} \triangleq \mathcal{E}\left\{\mathbf{H}_{u} \mathbf{H}_{u}{ }^{H}\right\}+\sigma_{n}^{2} \mathbf{I}_{N} . \quad 745$

The benefit of presenting the multiple-symbol differential 746 decision rule in the form of (4) is that the MSDD algorithm now 747 can be regarded as a "shortest vector problem", which may be 748 efficiently solved by sphere detection [61], [76].

749

According to the sphere decoding strategy [76], the specific 750 DPSK symbol vectors $\hat{\mathbf{S}}$, which are located within a sphere of 751 radius $R$, for which we have $\left\|\mathbf{U}^{u} \hat{\mathbf{S}}\right\|^{2} \leq R^{2}$ will be temporarily 752 regarded as potential candidates. Then, the squared Frobenius 753 norm $\left\|\mathbf{U}^{u} \hat{\mathbf{S}}\right\|^{2}$ will be employed as the new sphere radius. 754 Hence the sphere radius $R$ is further reduced. By contrast, 755 any DPSK symbol vector $\hat{\mathbf{S}}$ that violates the condition of 756 $\left\|\mathbf{U}^{u} \hat{\mathbf{S}}\right\|^{2} \leq R^{2}$ will be excluded from the search. This test is 757 repeated along with a gradually reduced sphere radius $R$, until 758 only the optimum candidate vector $\hat{\mathbf{S}}$ lies within the sphere. 759 This process is exemplified in Fig. 16, where an observa- 760 tion window of $N=5$ and a differentially-encoded 4-DPSK 761 differential-detection aided modulation scheme are employed. 762

Since the matrix $\mathbf{U}^{u}$ involved in (4) has an upper triangular 763 form, we may carry out the sphere decoding in a component- 764 wise manner. Bearing in mind that we have $\hat{\mathbf{S}}=[\hat{S}[1], \hat{S}[2], \ldots, 765$ $\hat{S}[N]]^{T}$, the squared Frobenius norm accounting only for the last 766 $(N-i+1)$ components of $\hat{\mathbf{S}}$, i.e. $[\hat{S}[i], \hat{S}[i+1], \ldots, \hat{S}[N]]^{T} 767$ is a fraction of the total squared Frobenius norm $\left\|\mathbf{U}^{u} \hat{\mathbf{S}}\right\|^{2}$ in (4). 768 Therefore, once the partial squared Frobenius norm of a legiti- 769 mate DPSK symbol vector $\hat{\mathbf{S}}$ has already exceeded the current 770 sphere radius $R_{\text {current }}^{2}$, it is logical that regardless of the first 771 $(i-1)$ components of $\hat{\mathbf{S}}$, i.e. regardless of $[\hat{S}[1], \hat{S}[2], \cdots, 772$ $\hat{S}[i-1]]^{T}$, its completed squared Frobenius norm $\left\|\mathbf{U}^{u} \hat{\mathbf{S}}\right\|^{2} 773$ must also exceed the current sphere radius $R_{\text {current }}^{2}$. This 774 implies that $M_{c}^{(i-1)}$ legitimate combinations of $\hat{\mathbf{S}}$ are justifi- 775 ably excluded from the search space even without completing 776 their associated FNCs. Consequently, the "Excluded Groups" 777 displayed in Fig. 16 are identified. This is also the major 778 reason why the MSDSD algorithm is capable of significantly 779 mitigating the decoding complexity of the MSDD algorithm, as 780 demonstrated in Fig. 12 of Section II-C.

As mentioned in Section I and detailed in [67] and [68], 782 the MSDSD algorithm formulated in (4) has also been applied 783 in cooperative communication scenarios. One of the critical 784 design issues elaborated on in [67] and [68] is that of combin- 785 ing the multiple relay-aided received signals $\left\{\mathbf{Z}_{u}\right\}_{u=1}^{U}$, which 786 correspond to the same source symbol sequence, but are trans- 787 mitted by $U$ users acting as RNs and experiencing different 788 channels. This problem was solved by Wang et al. [68] leading 789 to their multiple-path MSDSD algorithm. However, the size of 790 the critical matrix $\mathbf{U}$ of [68, (26)] rapidly increases with the 791 number of multipath components $U$; hence the implementation 792 of sphere detection based on the decision metric proposed in 793 $[68,(26)]$ becomes a challenge, when the number of multipath 794 


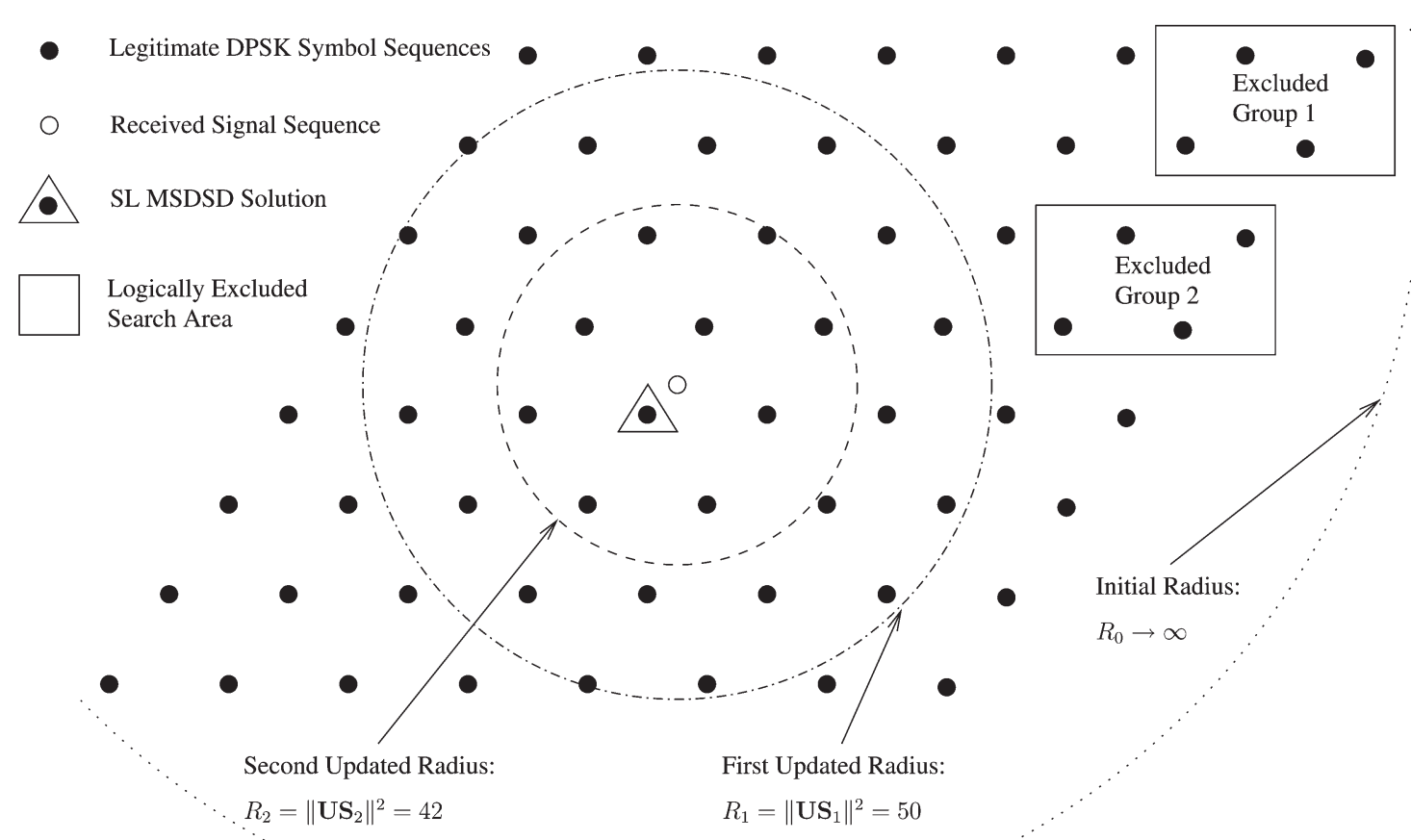

Fig. 16. Search space of the single-path MSDSD. Since we have $N=5$, there are $M_{c}^{N-1}=4^{4}=256$ legitimate DPSK symbol sequence $\hat{\mathbf{S}}$, but to avoid obfuscating details, we only depict part of them, which are explicitly labelled by 64 black dots. Then, the original signal sequence $\mathbf{S}$ will incur an amplitude attenuation and phase rotation of its component 4-DPSK symbols. Hence the received signal sequence represented by the hollow circle in the centre cannot fall onto any of the legitimate DPSK symbol sequences. Instead, it has a certain distance from the black dots. Thus, our MSDSD algorithm aims at finding the black dot that has the minimum distance from the hollow circle, which is deemed to be the original signal sequence $\mathbf{S}$.

$795 U$ increases. Thus, a new HDB relay-aided MSDSD algorithm 796 was devised in [69].

797 Since the received sequences $\left\{\mathbf{Z}_{u}\right\}_{u=1}^{U}$ are independent 798 Gaussian random vectors, the corresponding conditional PDF 799 can be written as

$$
\operatorname{Pr}\left(\left\{\mathbf{Z}_{u}\right\}_{u=1}^{U} \mid \mathbf{S}\right)=\prod_{u=1}^{U} \operatorname{Pr}\left(\mathbf{Z}_{u} \mid \mathbf{S}\right) .
$$

800 Upon substituting (2) into (5), (5) may be rewritten as

$$
\begin{aligned}
\operatorname{Pr}\left(\left\{\mathbf{Z}_{u}\right\}_{u=1}^{U} \mid \mathbf{S}\right) & =\left(\prod_{u=1}^{U} \eta_{u}\right) \\
& \times \exp \left(-\frac{1}{2} \sum_{u=1}^{U} \mathbf{Z}_{u}{ }^{H} \mathbf{\Phi}_{\mathbf{Z}_{u} \mathbf{Z}_{u}}^{-1} \mathbf{Z}_{u}\right),
\end{aligned}
$$

801 where the factor $\prod_{u=1}^{U} \eta_{u}$ is independent of $\mathbf{S}$. Hence, the relay802 aided MSDSD decision rule of finding the specific sequence $803 \hat{\mathbf{S}}=[\hat{S}[1], \hat{S}[2], \ldots, \hat{S}[N]]^{T}$ which maximises the conditional $804 \mathrm{PDF}$ of (6) is equivalent to selecting the particular transmitted 805 source signal sequence $\hat{\mathbf{S}}$, whose matrix sum is the smallest, 806 which is formulated as

$$
\begin{aligned}
\hat{\mathbf{S}} & =\underset{\mathbf{S} \in \chi}{\arg \min }\left\{\sum_{u=1}^{U} \mathbf{Z}_{u}{ }^{H} \mathbf{\Phi}_{\mathbf{Z}_{u} \mathbf{z}_{u}}^{-1} \mathbf{Z}_{u}\right\}, \\
& =\underset{\mathbf{S} \in \chi}{\arg \min }\left\{\sum_{u=1}^{U}\left\|\mathbf{U}^{u} \mathbf{S}\right\|^{2}\right\} .
\end{aligned}
$$

Then, we further define a $(U N \times N)$-element matrix $\mathbf{U}$ as

$$
\begin{aligned}
\mathbf{U} & =\left[\begin{array}{cccc}
\mathbf{U}_{1,1} & \mathbf{U}_{1,2} & \cdots & \mathbf{U}_{1, N} \\
0 & \mathbf{U}_{2,2} & \cdots & \mathbf{U}_{2, N} \\
\vdots & \vdots & \ddots & \vdots \\
0 & 0 & \cdots & \mathbf{U}_{N, N}
\end{array}\right]_{U N \times N}, \\
\mathbf{U}_{i, j} & =\left[\mathbf{U}_{i, j}^{1}, \mathbf{U}_{i, j}^{2}, \cdots, \mathbf{U}_{i, j}^{U}\right]^{T},
\end{aligned}
$$

where $\mathbf{U}_{i, j}$ is the specific vector component of $\mathbf{U}$ in row $i$ and 808 column $j, 1 \leq i, j \leq N$, and $\mathbf{U}_{i, j}^{u}$ is a specific element of $\mathbf{U}^{u} 809$ in row $i$ and column $j, 1 \leq i, j \leq N$, which was defined in 810 (4). Upon substituting (8) into (7), we finally arrive at the HDB 811 relay-aided MSDSD decision rule

$$
\hat{\mathbf{S}}=\underset{\mathbf{S} \in \chi}{\arg \min }\left\{\|\mathbf{U S}\|^{2}\right\}
$$

The size of the corresponding matrix $\mathbf{U}$ used in $[68,(26)]$ is 813 $\left(U N \times U^{2} N\right)$, which is cubically proportional to the number 814 of entities $U$. By contrast, the size of the newly devised matrix 815 U defined in (8) and involved in (9) is reduced to $(U N \times N), 816$ which only increases linearly with $U$. Thus, the HDB relay- 817 aided MSDSD algorithm may be decomposed into two steps: 818 first, we individually calculate each $\mathbf{U}^{u}$ contribution seen in (4) 819 according to the conventional single-path MSDSD algorithm; 820 then, we execute the relay-aided MSDSD based on the matrix 821 $\mathbf{U}$ of (4) according to the conventional single-path MSDSD 822 
823 algorithm; then, we execute the relay-aided MSDSD based on 824 the matrix $\mathbf{U}$ of (8), which is constituted by combining all the $825\left\{\mathbf{U}^{u}\right\}_{u=1}^{U}$ components.

\section{E. SDB Relay-Aided MSDSD}

827 As stated in Section I, relaxing hard-decision-based detection 828 to its soft-decision-based counterpart will achieve a substan829 tially improved energy-efficiency. To transform the HDB relay830 aided MSDSD to its SDB variant, we have to pay particular 831 attention to the a posteriori log-likelihood ratio (LLR) of the $832 \mu$ th bit $u[\mu]$, given $U$ simultaneously received signal streams 833 represented by $\left\{\mathbf{z}_{u}\right\}_{u=1}^{U}$, which may be formulated as

$$
L(u[\mu])=\ln \frac{\operatorname{Pr}\left(u[\mu]=b \mid\left\{\mathbf{z}_{u}\right\}_{u=1,2, \cdots, U}\right)}{\operatorname{Pr}\left(u[\mu]=\bar{b} \mid\left\{\mathbf{z}_{u}\right\}_{u=1,2, \cdots, U}\right)}, b \in\{0,1\},
$$

834 where $\bar{b}$ is the complement of $b$.

835 We will detail later in Section IV-B that the DN will receive 836 the pair of despread signal streams $\mathbf{z}_{s}^{l}$ and $\mathbf{z}_{r_{0}}^{l+1}$, which corre837 spond to the same SN's transmitted signal stream $\mathbf{S}^{l}$. Hence, for 838 example, we can substitute $\mathbf{z}_{s}^{l}$ and $\mathbf{z}_{r_{0}}^{l+1}$ into (10) as $\left\{\mathbf{z}_{u}\right\}_{u=1,2}$. 839 As a result, (10) can be rewritten with the aid of Bayes' 840 formula as

$$
\begin{aligned}
L(u[\mu]) & =\ln \frac{\operatorname{Pr}\left(u[\mu]=b \mid \mathbf{z}_{s}^{l}, \mathbf{z}_{r_{0}}^{l+1}\right)}{\operatorname{Pr}\left(u[\mu]=\bar{b} \mid \mathbf{z}_{s}^{l}, \mathbf{z}_{r_{0}}^{l+1}\right)} \\
& =\ln \frac{\sum_{\mathbf{V} \in \chi_{: u[\mu]=b}} \operatorname{Pr}\left(\mathbf{z}_{s}^{l} \mid \mathbf{V}\right) \operatorname{Pr}\left(\mathbf{z}_{r_{0}}^{l+1} \mid \mathbf{V}\right) \operatorname{Pr}(\mathbf{V})}{\sum_{\mathbf{V} \in \chi_{: u[\mu]=\bar{b}}} \operatorname{Pr}\left(\mathbf{z}_{s}^{l} \mid \mathbf{V}\right) \operatorname{Pr}\left(\mathbf{z}_{r_{0}}^{l+1} \mid \mathbf{V}\right) \operatorname{Pr}(\mathbf{V})},
\end{aligned}
$$

841 where the information symbol vector $\mathbf{V}$ consists of $\left(T_{b}-1\right)$ 842 QPSK symbols. The relationship of the symbol-vector $\mathbf{V}$ and $843 \mathbf{S}^{l}$ is also clarified in (1). Furthermore, $\chi_{: u[\mu]=b}$ represents 844 the set of $\left(M_{c}^{T_{b}-1} / 2\right)$ legitimate transmitted vectors $\mathbf{V}$, whose $845 \mu^{t h}$ bits are constrained to $u[\mu]=b$, and similarly, $\chi_{: u[\mu]=\bar{b}}$ is 846 defined as the set corresponding to $u[\mu]=\bar{b}$.

847 The next step is that of substituting the squared Frobenius 848 norm of $\left\|\mathbf{U}^{u} \mathbf{S}\right\|^{2}$ shown in (4) into (11), where we equivalently 849 represent the probabilities seen in (11) in terms of their asso850 ciated squared Frobenius norms. Similar manipulations can be 851 found in [66]. In more detail, according to (2), (3) and (4), we 852 arrive at

$$
\begin{gathered}
\operatorname{Pr}\left(\mathbf{z}_{s}^{l} \mid \mathbf{V}\right) \propto \exp \left\{-\left\|\mathbf{U}^{s} \mathbf{S}^{l}\right\|^{2}\right\}, \\
\operatorname{Pr}\left(\mathbf{z}_{r_{0}}^{l+1} \mid \mathbf{V}\right) \propto \exp \left\{-\left\|\mathbf{U}^{r_{0}} \mathbf{S}^{l}\right\|^{2}\right\} .
\end{gathered}
$$

853 The definition of the matrix $\mathbf{U}^{s}$ or $\mathbf{U}^{r_{0}}$ is similar to that 854 stipulated below (4). More particularly, the matrix $\mathbf{U}^{s}$ is related 855 to $\mathbf{z}_{s}^{l}$, while the matrix $\mathbf{U}^{r_{0}}$ is related to $\mathbf{z}_{r_{0}}^{l+1}$. Hence, based 856 on (11) and (12) and invoking the "sum-max" approximation as well as replacing $\mathbf{S}^{l}$ by the simplified notation of $\mathbf{S}$, the 857 a posteriori LLR of $u[\mu]$ is further approximated by

$$
\begin{aligned}
& L(u[\mu]) \\
& \approx \ln \frac{\max _{\mathbf{V} \in \chi_{: u[\mu]=b}} \exp \left\{-\left\|\mathbf{U}^{s} \mathbf{S}\right\|^{2}-\left\|\mathbf{U}^{r_{0}} \mathbf{S}\right\|^{2}+\ln \operatorname{Pr}(\mathbf{V})\right\}}{\max _{\mathbf{V} \in \chi_{: u[\mu]=\bar{b}}} \exp \left\{-\left\|\mathbf{U}^{s} \mathbf{S}\right\|^{2}-\left\|\mathbf{U}^{r_{0}} \mathbf{S}\right\|^{2}+\ln \operatorname{Pr}(\mathbf{V})\right\}} \\
& =\underbrace{\left(\left\|\mathbf{U}^{s} \hat{\mathbf{S}}_{\mathrm{MAP}}^{b}\right\|^{2}+\left\|\mathbf{U}^{r_{0}} \hat{\mathbf{S}}_{\mathrm{MAP}}^{b}\right\|^{2}-\ln \operatorname{Pr}\left(\hat{\mathbf{V}}_{\mathrm{MAP}}^{b}\right)\right)}_{\text {MAP-MSDSD } \in \chi_{: u[\mu]=b}} \\
& +\underbrace{\left(\left\|\mathbf{U}^{s} \hat{\mathbf{S}}_{\mathrm{MAP}}^{\bar{b}}\right\|^{2}+\left\|\mathbf{U}^{r_{0}} \hat{\mathbf{S}}_{\mathrm{MAP}}^{\bar{b}}\right\|^{2}-\ln \operatorname{Pr}\left(\hat{\mathbf{V}}_{\mathrm{MAP}}^{\bar{b}}\right)\right)}_{\text {MAP-MSDSD } \in \chi: u[\mu]=\bar{b}}
\end{aligned}
$$

The last step is that of performing the calculation of $L(u[\mu]) 859$ of (13) with the aid of the sphere detection process. The 860 evaluation of the a posteriori LLR of $L(u[\mu])$ in (13) may be 861 summarized as follows:

863

1. Let $\hat{\mathbf{S}}_{\text {MAP }}$ denote one of the legitimate differentially en- 864 coded DQPSK symbol vectors $\mathbf{S}$, which minimizes the 865 term $\left\{\left\|\mathbf{U}^{s} \mathbf{S}\right\|^{2}+\left\|\mathbf{U}^{r_{0}} \mathbf{S}\right\|^{2}-\ln \operatorname{Pr}(\mathbf{V})\right\}$ involved in the 866 numerator of (13). Then $\hat{\mathbf{V}}_{\text {MAP }}$ represents the correspond- 867 ing QPSK symbol vector, which is uniquely identified by 868 $\hat{\mathbf{S}}_{\text {MAP. }}$. The symbol vector $\hat{\mathbf{S}}_{\text {MAP }}$ may be obtained by im- 869 plementing a specific sphere detection algorithm, which 870 is an amalgam of the maximum a posteriori algorithm 871 called MAP-MSDSD in [62] and of the Relay-Aided 872 MSDSD algorithm described in Section II-D.

873

2. Employ $\hat{\mathbf{V}}_{\text {MAP as }} \hat{\mathbf{V}}_{\text {MAP }}^{b}$ and $\hat{\mathbf{S}}_{\mathrm{MAP}}$ as $\hat{\mathbf{S}}_{\mathrm{MAP}}^{b}$. Correspond- 874 ingly, the detected value of the $\mu$ th bit of $\hat{\mathbf{V}}_{\text {MAP }}$ is assigned 875 to the variable $b$ in (13). As a result, both the value of $\bar{b}$ and 876 the reduced-size search space of $\chi_{: u[\mu]=\bar{b}}$ are determined. 877

3. Implement a constrained MAP-MSDSD algorithm for the 878 sake of seeking $\hat{\mathbf{V}}_{\text {MAP }}^{\bar{b}}$ and $\hat{\mathbf{S}}_{\mathrm{MAP}}^{\bar{b}}$, which is similar to the 879 search process implemented during Step.1. However, this 880 time, the search space is reduced to $\chi_{: u[\mu]=\bar{b}}$. Correspond- 881 ingly, the associated $\hat{\mathbf{V}}_{\text {MAP }}^{\bar{b}}$ and $\hat{\mathbf{S}}_{\text {MAP }}^{\bar{b}}$ is obtained. 882

4. On substituting the resulting vectors $\hat{\mathbf{V}}_{\text {MAP }}^{b}, \hat{\mathbf{S}}_{\mathrm{MAP}}^{b}, \hat{\mathbf{V}}_{\mathrm{MAP}}^{\bar{b}} 883$ and $\hat{\mathbf{S}}_{\mathrm{MAP}}^{\bar{b}}$ into (13), the a posteriori LLR of $u[\mu]$ is 884 obtained.

\section{Successive AF Relaying Aided DS-CDMA UPLINK}

\section{A. Successive AF Relaying Aided Single-User DS-CDMA}

Uplink: Spread-Despread Strategy and Employing

\section{Relay-Aided MSDSD}

In this section, we focus our attention on the successive 891 AF relaying aided single-user DS-CDMA uplink, where the 892 specific DS-CDMA spreading-despreading strategy designed 893 for appropriately suppressing the CCI is highlighted.

We consider the successive relaying induced CCI problem 895 reported in [46] and [34], which is imposed by the signals 896 directly transmitted by the SN and the signals forwarded by one 897 of the two RNs, both of which are assumed to be simultaneously 898 received at the DN, as depicted in "Phase 1" and "Phase 2" 899 of Fig. 5. Hence, the algorithm discussed in [77] constitutes 900 


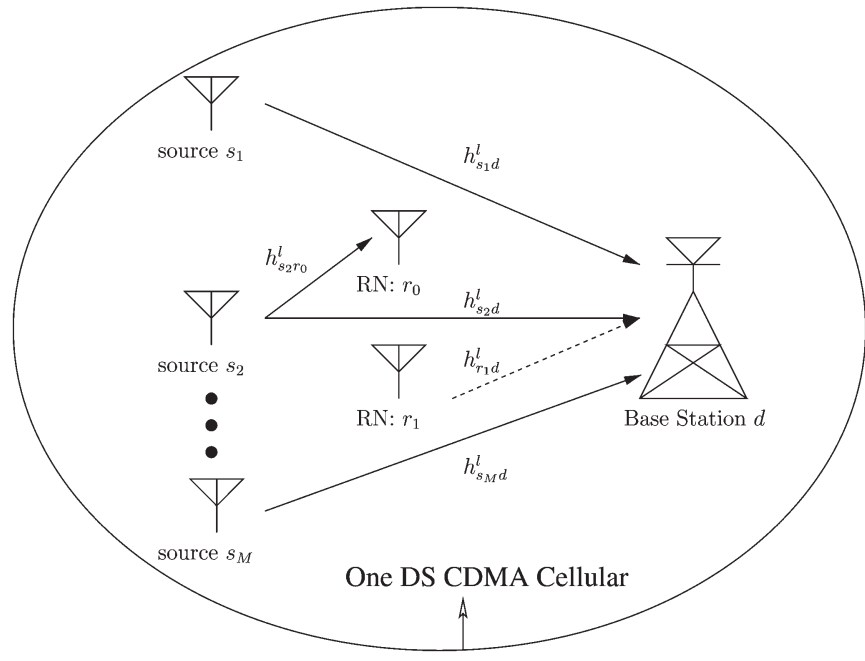

Fig. 17. A simplified uplink diagram of successive AF relaying aided DSCDMA, assuming that the successive relaying aided cooperative sub-system is embedded in the middle.

901 a beneficial technique for extracting the different components 902 of the resulting composite signal. Inspired by this concept, the 903 DS-CDMA technique was considered in [77] for suppressing 904 the successive relaying induced CCI, which however has to 905 be further developed for employment in the SRAN of [46], 906 seen in Fig. 18.

907 Consequently, based on [69], a specific successive AF re908 laying aided DS-CDMA uplink is portrayed in Fig. 17, where $909 s_{n}, n \in\{1,2, \ldots, M\}$ represent the mobile stations (MSs) and $910 h_{a b}^{l}, a, b \in\left\{s_{n}, r_{0}, r_{1}, d\right\}$ represent the CIRs spanning from 911 node $a$ to node $b$ during the $l^{\text {th }}$ frame. In [69], it was assumed 912 that some of the idle MSs are willing to act as the relays $r_{0}, r_{1}$ 913 and they exclusively assist one of the $M$ users seen on the left 914 hand side of Fig. 17 based on the successive relaying protocol 915 of [46]. It was also assumed in [69] that the impact of the 916 successive relaying induced IRI reported in [46] is negligible, 917 in other words, relay $r_{0}$ shown in Fig. 17 is assumed to receive 918 no interference from relay $r_{1}$ and vice versa. Furthermore, the 919 signals are assumed to be perfectly synchronised, for example 920 using the solution of [78]. The path-loss effects were ignored in 921 [69] for simplicity. Assuming that the overall transmit power of 922 a single-link direct-transmission based system is $P_{\text {total }}$, for the 923 sake of a fair comparison with classic direct communications 924 using no relays, we explicitly stipulate that the transmit power 925 of every node involved in the SRAN obeys the relationship of $926 P_{s}+P_{r_{i}}=P_{\text {total }}, i \in\{0,1\}$ and $P_{s}=P_{r_{i}}, i \in\{0,1\}$, where $927 P_{a}$ denotes the transmit power of node $a$. To improve the prac928 ticability of the solutions reviewed, standard frame-by-frame 929 based transmissions routinely employed in realistic communi930 cation networks are considered here. We will use a single phase 931 depicted in Fig. 5 to transmit a single frame, i.e. the length of 932 a transmission frame is identical to the duration of a phase. 933 According to the specific structure of the SRAN described 934 in Section II-A, when employing frame-based transmission, a 935 specific codeword forwarded by the RN will arrive at the DN a 936 frame period later with respect to the directly received replica 937 transmitted by the SN. Hence the system delay is proportional 938 to the frame length $L$.
Then, a specific DS-CDMA spreading-despreading strategy 939 may be invoked [69], which is described below:

1) All the frames are divided into even frames $(l=0,2,4, \cdots) 942$ and odd frames $(l=1,3,5, \cdots)$. A pair of pseudo-noise 943 (PN) sequences, namely $\mathbf{C}_{0}$ and $\mathbf{C}_{1}$ chosen from the en- 944 tire spreading sequence family is employed for spreading 945 the signals transmitted by the SN during even frames and 946 odd frames, respectively. For example, as illustrated in 947 Fig. 18, the signal transmitted by the SN $s$ during "Phase 948 0", namely $S^{0}[k]$ is spread by the PN sequence $\mathbf{C}_{0}$. Then, 949 during the consecutive odd phase "Phase 1", the signal 950 $S^{1}[k]$ is spread by another PN sequence $\mathbf{C}_{1}$. Then, the PN 951 sequence employed by the $\mathrm{SN} s$ during the consecutive 952 even phase "Phase 2" is switched to $\mathbf{C}_{0}$ again. 953

2) Thus, this spreading scheme guarantees that the two dif- 954 ferent components of the $k^{t h}$ signal $\mathbf{y}^{l}[k]$ received at the 955 DN during the $l^{\text {th }}$ frame, namely those which correspond 956 to the SN's transmitted signal and to the RN's forwarded 957 signal, respectively, are always spread by different PN 958 sequences. This can be confirmed in "Phase 2" of Fig. 18, 959 where the signal $S^{2}[k]$ broadcast by the SN $s$ and the 960 signal $S^{1}[k]$ forwarded by the RN $r_{1}$ interfere with each 961 other at the DN $d$, which leads to CCI. Based on the 962 above-mentioned spreading strategy, the signals $S^{2}[k] 963$ and $S^{1}[k]$ are spread by different PN sequences, namely 964 by $\mathbf{C}_{0}$ and $\mathbf{C}_{1}$, respectively.

965

3) Hence, if the matched filter used for despreading $\mathbf{y}^{l}[k] 966$ is matched to the waveform $\mathbf{C}_{0}$, the signal component 967 of $S^{2}[k]$ can be extracted from the received signal $\mathbf{y}^{l}[k] .968$ Meanwhile, when the matched filter is matched to the 969 waveform $\mathbf{C}_{1}$, the signal component of $S^{1}[k]$ can be 970 recovered.

Based on the above-introduced specific DS-CDMA spreading- 972 despreading strategy, at the DN, the different components of 973 the received signal $\mathbf{y}^{l}[k]$ can be extracted by appropriately con- 974 figuring the matched filter matched to the different spreading 975 codes. If we arrange for the system to obey the relationships of 976 $i \equiv[(l+1) \bmod 2] ; \bar{i} \equiv[l \bmod 2]$ and the matched filter used 977 for despreading $\mathbf{y}^{l}[k]$ is matched to the waveform $\mathbf{C}_{\bar{i}}$, the signal 978 directly transmitted by the SN will contribute the main compo- 979 nent of the despread signal, while the RN's forwarded signal 980 becomes the interference of $I_{r_{i} d}^{l}[k]$. The associated output of 981 the chip-waveform matched-filter is represented by $z_{s}^{l}[k]$. Let 982 us define $\mathbf{Z}_{s}^{l}=\left[z_{s}^{l}[1], z_{s}^{l}[2], \ldots, z_{s}^{l}[N]\right]^{T}$, which corresponds to 983 a length- $N M_{c}$-ary DPSK symbol vector broadcast by the SN 984 in Fig. 18 during the $l$ th frame, namely to $\mathbf{S}^{l}$.

985

The other symbol vector $\mathbf{S}^{l}$ will be forwarded by the RN 986 $r_{\bar{i}}$, which arrives at the DN $d L$ symbol periods later in the 987 consecutive $(l+1)^{s t}$ frame. Similarly, during the $(l+1)^{s t} 988$ frame, we configure the filter to match the waveform of $\mathbf{C}_{\bar{i}} .989$ Consequently, the despread signal extracted from $\mathbf{y}^{l+1}[k]$ is 990 dominated by the RN's forwarded signal, while the signal 991 directly transmitted by the SN becomes the CCI of $I_{s d}^{l+1}[k]$. This 992 despread signal is represented by $z_{r_{\bar{i}}}^{l+1}[k]$. Let us also define 993 $\mathbf{Z}_{r_{\bar{i}}}^{l+1}=\left[z_{r_{\bar{i}}}^{l+1}[1], z_{r_{\bar{i}}}^{l+1}[2], \ldots, z_{r_{\bar{i}}}^{l+1}[N]\right]^{T}$.

After completing the despreading of the signals received at the 995 DN $d$, the next step is to invoke the HDB relay-aided MSDSD 996 


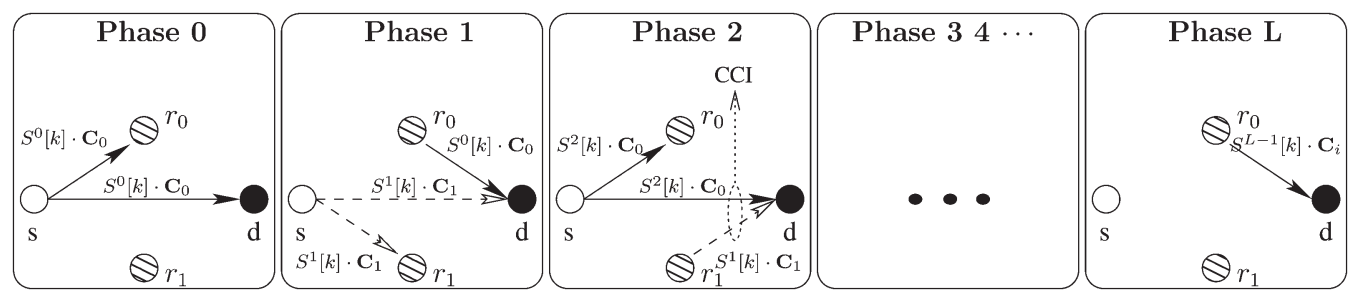

Fig. 18. The specific DS-CDMA spreading strategy, where $S^{l}[k]$ represents the $k^{t h}$ DPSK symbol of the $l^{t h}$ signal frame. The CCI problem is highlighted in "Phase 2". This figure is obtained by applying the above-mentioned DS-CDMA spreading strategy to the relaying regime of Fig. 5.

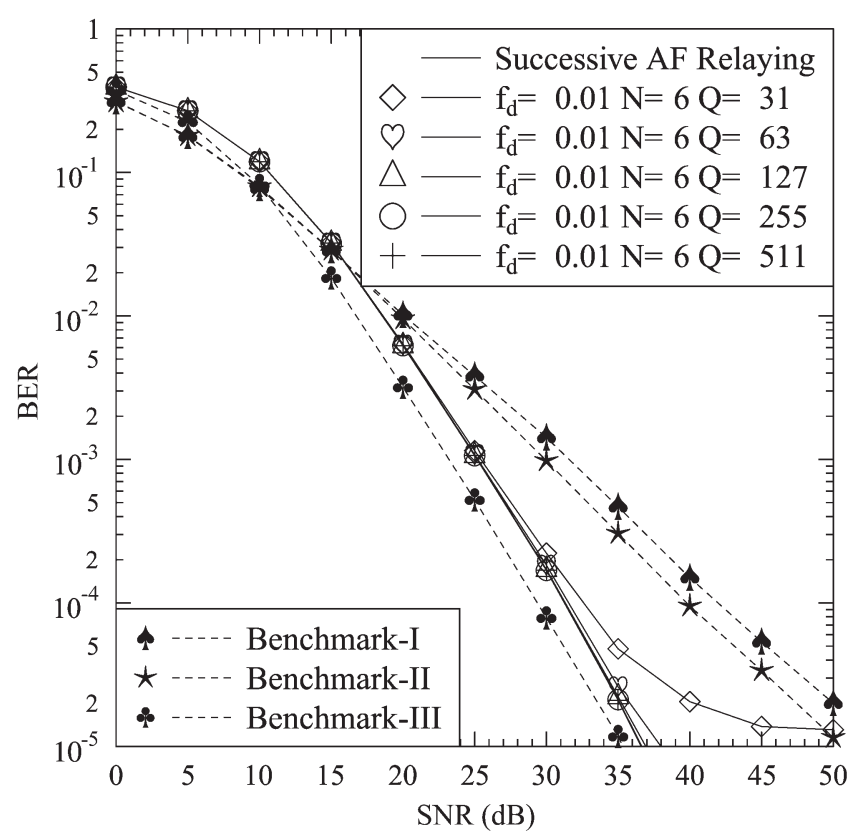

Fig. 19. BER versus SNR of the successive AF relaying aided single-user DS-CDMA uplink of Fig. 17 employing the HDB relay-aided MSDSD algorithm and using various spreading factors of $Q$. The interpretations of the three benchmark schemes employed for comparison are given in Table IX.

997 algorithm of Fig. 16 introduced in Section II-D for carrying 998 out the noncoherent differential detection at the DN $d$. Clearly, 999 according to the principle described in Section II-D based 1000 on [62] and [79], we can directly implement the HDB relay1001 aided MSDSD algorithm of [69] by using $\mathbf{Z}_{s}^{l}$ and $\mathbf{Z}_{r_{\bar{i}}}^{l+1}$ as the $1002 \mathbf{Z}_{u}$ components in (7). Consequently, the relay-aided MSDSD 1003 detection process of the successive AF relaying aided single1004 user DS-CDMA uplink shown in Fig. 17 becomes feasible.

\section{B. Successive AF Relaying Aided Single-User DS-CDMA 1006 Uplink: BER Versus Complexity Performance}

1007 Observe in Fig. 13 that conventional two-phase AF relaying 1008 operating with the aid of the multiple-path MSDSD algorithm 1009 of [68] incurs a severe 50\% throughput loss problem. In con1010 trast, the successive AF relaying regime of [46] addressed in 1011 Section III-A is capable of recovering the 50\% throughput loss. 1012 However, this throughput improvement is achieved at the cost 1013 of imposing CCI plus the extra amplified and faded noise com1014 ponent on the received signals, which may erode the achievable 1015 BER performance gain. This BER performance loss is charac1016 terized in Fig. 19. The system parameters used in the simulation 1017 results of this section for the sake of substantiating our dis1018 cussions are based on [69] and are summarized in Table VIII.
TABLE VIII SYSTEM PARAMETERS

\begin{tabular}{|l|r|}
\hline Scenario & Single-User DS-CDMA Uplink \\
\hline System Regime & Successive AF Relaying \\
\hline Channel Model & $f_{d} \in\{0.01,0.06\}$ \\
\hline Normalized Doppler Frequency & Gold Sequence \\
\hline PN sequence & Time-Selective Flat Rayleigh Fading \\
\hline Spreading Factor & Differential QPSK \\
\hline Modulation Scheme & HDB Relay-Aided MSDSD \\
\hline Demodulation Scheme & $N \in\{3,4,6,8,10,12\}$ \\
\hline MSDSD Observation Window Size & \\
\hline
\end{tabular}

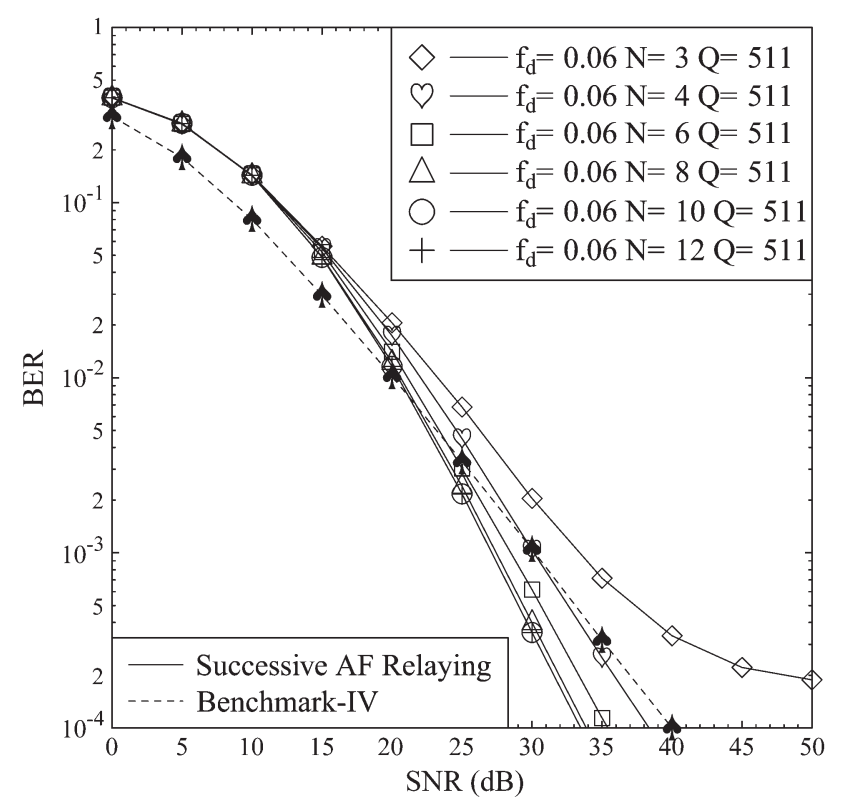

Fig. 20. Effects of the MSDSD observation window size on the BER performance of the successive AF relaying aided DS-CDMA uplink of Fig. 17 using the parameters of Table VIII.

Specifically, in all the experiments based on the successive 1019 AF relaying system characterized in Fig. 19, the normalized 1020 Doppler frequency is fixed to $f_{d}=0.01$ and the observation 1021 window size of the HDB relay-aided MSDSD is fixed to $N=6.1022$

Firstly, the impact of the successive relaying induced CCI 1023 is quantified, since it significantly affects the system's BER 1024 performance. As shown in Fig. 19, increasing the DS-CDMA 1025 spreading factor (SF) $Q$ mitigates the influence of the CCI. Nev- 1026 ertheless, an error floor is encountered for a SF of 31 between 1027 BER of $10^{-4}$ and of $10^{-5}$. No significant BER improvement 1028 can be attained upon increasing the SF beyond 63, but never- 1029 theless a SF of 511 will be adopted in our forthcoming investi- 1030 gations for minimizing the influence of interference in adverse 1031 propagation conditions, as justified at a later stage in the context 1032 of Fig. 20. Then, the relay-aided MSDSD assisted successive 1033 
TABLE IX

DEFINITIONS OF THE BENCHMARKS EMPLOYED IN FIGS. 19 AND 20

\begin{tabular}{|l|llr|}
\hline Benchmark-I & single-user scenario dispensing with relaying & MSDSD [62] & $f_{d}=0.01 N=6$ \\
\hline Benchmark-II & single-user scenario dispensing with relaying & coherent DQPSK & $f_{d}=0.01$ \\
\hline Benchmark-III & single relay-aided two-phase cooperation & multi-path MSDSD [68 $]$ & $f_{d}=0.01 N=6$ \\
\hline Benchmark-IV & single-user scenario dispensing with relaying & coherent DQPSK & $f_{d}=0.06$ \\
\hline
\end{tabular}

$1034 \mathrm{AF}$ system using $\mathrm{SF}=511$ requires an approximately $2 \mathrm{~dB}$ 1035 higher transmit power for achieving the target BER of $10^{-4}$ 1036 compared to benchmark-III of Table IX, i.e. in comparison to 1037 its counterpart shown in Fig. 13. As stated early in this section, 1038 the associated performance degradation seen in Fig. 19 is 1039 attributable to two main reasons: firstly, to the successive1040 relaying-induced CCI between the transmitted signals of the $1041 \mathrm{SN}$ and RN; secondly, to the noise component amplified and 1042 forwarded by the RN $r_{i}$, which is imposed on the despread 1043 source signal $z_{s}^{l}[k]$ introduced in Section III-A, but would never 1044 appear in the Source-to-Destination link of benchmark-III.

1045 According to the comparison of the successive AF relaying 1046 system and benchmark-III, we may argue that the successive 1047 AF relaying system doubles the spectral efficiency with re1048 gard to benchmark-III without substantially degrading the BER 1049 performance. On the other hand, the successive AF relay1050 ing system substantially outperforms the conventional single1051 user direct-transmission based schemes operating without RNs, 1052 which includes the benchmark-I and benchmark-II. Hence the 1053 successive AF relaying system is capable of striking the most 1054 attractive trade-off between the achievable throughput and the 1055 attainable BER performance amongst all the schemes listed 1056 in Table IX.

1057 As stated in Section II-C, apart from increasing the spatial 1058 diversity order for the sake ofreducing the detrimental impact 1059 of time-selective fading channels, increasing the observation 1060 window size of the MSDSD algorithm is another beneficial 1061 method, which results in an improved time diversity. However, 1062 when the window size is extended, the complexity imposed in1063 creases rapidly. To assess the trade-offs between the attainable 1064 time diversity and the system's complexity, simulation results 1065 are provided in Figs. 20 and 21, where the normalized Doppler 1066 frequency is fixed to $f_{d}=0.06$, the spreading factor is fixed to $1067 Q=511$ and the observation window size $N$ increases from 10683 to 12 . The remaining parameters are configured according to 1069 Table VIII again.

1070 As expected, an increased performance gain is attained when 1071 the window size increases from $N=3$ to 12 , as shown in 1072 Fig. 20. However, no further significant performance gain may 1073 be attained, once the window size reached $N=8$, despite in1074 vesting substantially increased search complexity, as quantified 1075 in terms of the number of multiplications required for decoding 1076 a single symbol. This is particularly so in the low-SNR region, 1077 as shown in Fig. 21. In practical applications, the BER should 1078 be lower than $10^{-3}$. In other words, according to Fig. 20, 1079 the new system performs well for SNRs above $30 \mathrm{~dB}$. In 1080 this SNR range of Fig. 21, the complexity difference is no 1081 longer significant between the different window sizes. This 1082 justifies our previous statements, arguing that a window size 1083 of $N=8$ could be a meritorious choice, which has a moderate

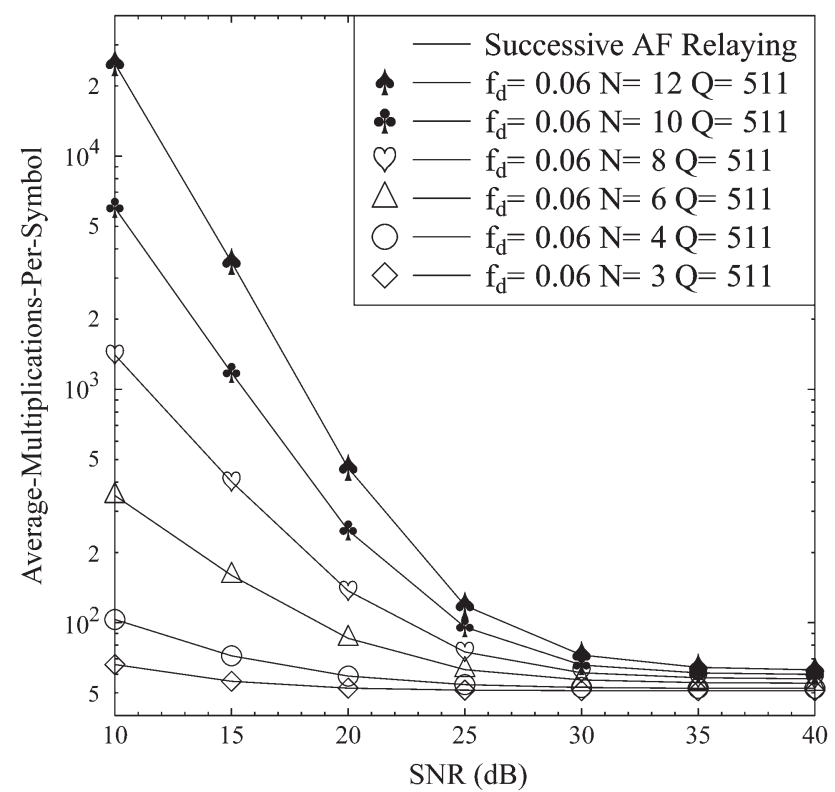

Fig. 21. Complexity versus SNR of the successive AF relaying aided DSCDMA uplink of Fig. 17 upon varying the observation window size of the HDB relay-aided MSDSD algorithm employed. The system parameters are summarized in Table VIII.

complexity, whilst achieving the best BER performance in the 1084 context of the parameters considered.

C. Successive AF Relaying Aided Multi-User DS-CDMA Uplink: Consider CCI, IRI and MAI

In this section, we focus our attention on the successive AF 1088 relaying aided multi-user DS-CDMA uplink, where a more 1089 realistic scenario is considered, which takes both the IRI as 1090 well as the multiple-access interference (MAI) into account. 1091 Furthermore, in the spirit of Section III-A, these successive- 1092 relaying-induced interferences will be suppressed by relying on 1093 the classic DS-CDMA principle upon assigning unique, link- 1094 specific spreading codes to the potentially interfering links. 1095 Naturally, this implies that the orthogonal time-slots used in 1096 [46] are replaced by unique, link-specific CDMA spreading 1097 codes at the cost of imposing a soft-limit on the number of users 1098 that may be supported, given the limited number of spreading 1099 codes.

After extending the single-user DS-CDMA uplink to the 1101 more realistic multi-user scenario, the prototype system shown 1102 in Fig. 17 is correspondingly replaced by a more generalized 1103 network topology, which is portrayed in Fig. 22, where the MS 1104 $s$ roaming close to the edge of the DS-CDMA cell activates 1105 the SRAN regime to improve its communication quality. In 1106 


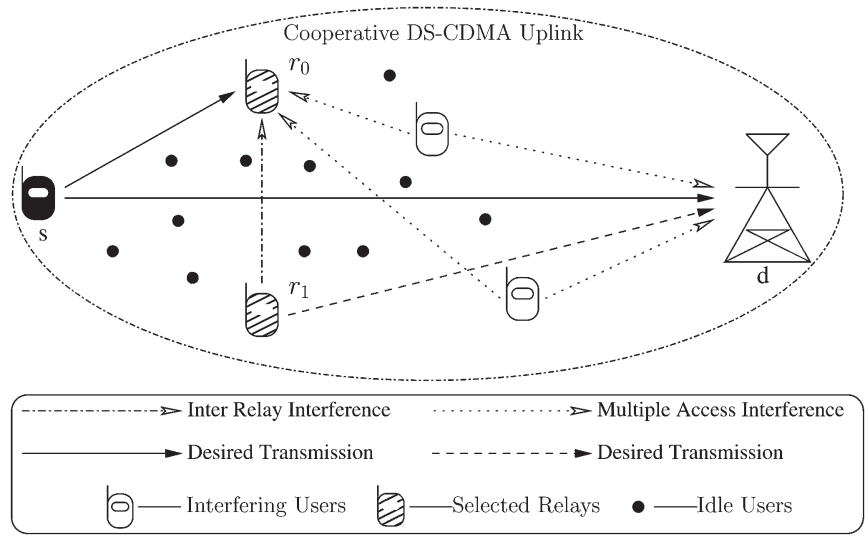

Fig. 22. The successive AF relaying aided multi-user DS-CDMA uplink topology.

1107 Section III-A, the path-loss effect encountered in realistic 1108 systems is ignored for the sake of simplifying the analysis. 1109 By contrast, to improve the practicality of the investigations, 1110 the path-loss effects are also taken into account from this 1111 section onwards. As detailed in [80], the average path-loss 1112 reduction gain of the link spanning from node $a$ to node $1113 b$ with respect to the Source-to-Destination link is given by $1114 G_{a b}=\left(D_{s d} / D_{a b}\right)^{\alpha},(a, b) \in\left\{s, r_{0}, r_{1}, d\right\}$, where the notation $1115 D_{a b}$ represents the distance between node $a$ and node $b$. 1116 Throughout this paper, the path-loss exponent is fixed to $\alpha=3$ 1117 for representing a typical urban area. To simplify our analysis, 1118 we assume that the SRAN has a symmetric topology, which 1119 implies that $D_{s r_{0}}, D_{r_{0} d}, G_{s r_{0}}$ and $G_{r_{0} d}$ are identical to $D_{s r_{1}}$, $1120 D_{r_{1} d}, G_{s r_{1}}$ and $G_{r_{1} d}$, respectively.

1121 1) Noise Accumulation Problem: In the scenario specified in 1122 Section III-A, where the successive relaying induced IRI was 1123 not considered, we did not incur the noise accumulation prob1124 lem either. However, when considering the IRI encountered 1125 by our system illustrated in Fig. 22, the noise accumulation 1126 problem detailed in this section will occur owing to directly 1127 employing the classic AF protocol in the SRAN, where the 1128 RN simply amplifies its received signal before retransmitting 1129 it. This noise accumulation problem is visualized in Fig. 23. 1130 In more detail, the noise accumulation process portrayed in 1131 Fig. 23 may be summarised as follows:
1) Observe in "Fig. 23(a) Phase 0" that the $\mathrm{RN} r_{0}$ receives an AWGN contribution, namely "Noise 0 ". Hence, at this moment, the AN imposed at the $\mathrm{RN} r_{0}$ only has a single component: "Noise 0".

2) Observe in "Fig. 23(b) Phase 1" that the RN $r_{0}$ simply amplifies all the signal components received during "(a) Phase 0" and then transmits them. Hence, the noise component "Noise 0" is also forwarded to the RN $r_{1}$ as part of the IRI. Simultaneously, a new AWGN contribution generated during "Fig. 23(b) Phase 1", namely "Noise 1" is further imposed on the $\mathrm{RN} r_{1}$. Hence, during "Fig. 23(b) Phase 1", the AN imposed at $r_{1}$ increases to two components: "Noise 0 " and "Noise 1".

3) Observe in "Fig. 23(c) Phase 2" that the AN consisting of "Noise 0" and "Noise 1", which is imposed on the RN $r_{1}$

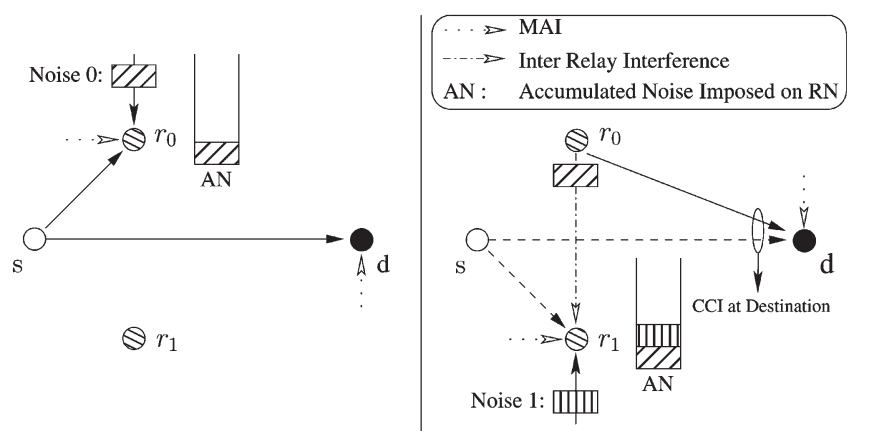

(a)

(b)

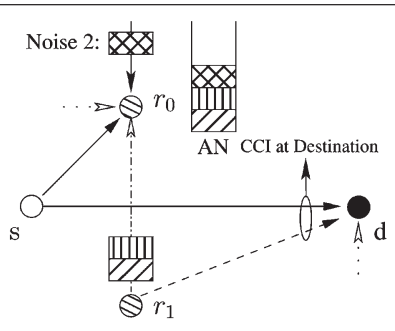

(c)

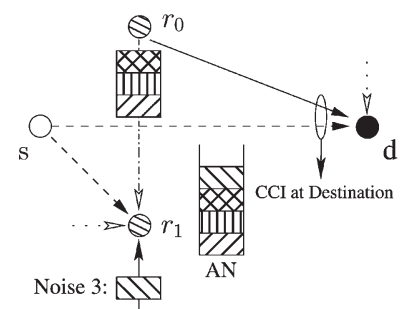

(d)

Fig. 23. The accumulated noise (AN) imposed on the RNs during the successive AF relaying process. (a) Phase 0. (b) Phase 1. (c) Phase 2. (d) Phase 3.

during the last phase is further forwarded to the $\mathrm{RN} r_{0} .1148$ Simultaneously, a new AWGN contribution generated 1149 during "Fig. 23(c) Phase 2", namely "Noise 2" is also 1150 imposed on the RN $r_{0}$. Hence, now, the AN imposed 1151 on $r_{0}$ has three components: "Noise 0", "Noise 1" and 1152 "Noise 2".

4) Owing to the same mechanism as stated above, the AN 1154 imposed on the $\mathrm{RN} r_{1}$ will have four components during 1155 "Fig. 23(d) Phase 3". Obviously, the accumulated noise 1156 imposed on the RNs will continue to increase upon 1157 continuing the successive AF relaying transmissions.

1158

Based on the assumption that the power $P_{s}^{l}$ of the SN's 1159 broadcast signal, the power $P_{\mathrm{MAI}}^{l}$ of the MAI as well as the 1160 SNR remain constant for the different frames, and proceeding 1161 backwards from the $(l+1)^{s t}$ to the $2^{\text {nd }}$ frame of Fig. 23, the 1162 variance of the total recursively accumulated noise component 1163 in the transmit power $P_{r_{i}}^{l+1}$ of the $\mathrm{RN} r_{i}$ has been evaluated in 1164 [81], which is approximated as

$$
\begin{aligned}
\operatorname{Var}\left[N_{\text {total }}\right] & \approx \sigma^{2} f_{A M_{r_{i}}}^{2} \lim _{l \rightarrow \infty} \sum_{n=0}^{l-1}\left(f_{A M_{r_{i}}}^{2} G_{r_{\bar{i}} r_{i}}\right)^{n} \\
& =\frac{\sigma^{2} f_{A M_{r_{i}}}^{2}}{1-f_{A M_{r_{i}}}^{2} G_{r_{\bar{i}} r_{i}}},
\end{aligned}
$$

where $\sigma^{2}$ is the variance of the AWGN. This implies that all 1166 the noise generated in different frames at the different relays 1167 will be consistently scaled and accumulated during the trans- 1168 mission process of the classic AF based SRAN. Quantitatively, 1169 they impose an extra noise component having a variance of 1170 
$1171 \sigma^{2} f_{A M_{r_{i}}}^{2} G_{r_{i} d} \cdot\left(1 / 1-f_{A M_{r_{i}}}^{2} G_{r_{\bar{i}} r_{i}}\right)$ on the $\mathrm{DN},{ }^{8}$ which cannot 1172 be mitigated by the single despreading operation at the DN.

1173 2) Interference Suppression Regime: Let $\gamma_{01}$ denote the 1174 cross-correlation (CCL) of the pair of PN sequences $\mathbf{C}_{i}, i \in$ $1175\{0,1\}$ illustrated in Fig. 18. Observe at "Phase 0" of Fig. 18 that 1176 the signal component $S^{0}[k] \cdot \mathbf{C}_{0}$ is received at $\mathrm{RN} r_{0}$. After 1177 despreading it at the DN $d$ by $\mathbf{C}_{1}^{T}$ at the ensuing "Phase 1", this 1178 signal component becomes $S^{0}[k] \cdot \mathbf{C}_{0} \cdot \mathbf{C}_{1}^{T}=S^{0}[k] \gamma_{01}$, which 1179 implies that the interfering signal $S^{0}[k]$ is effectively mitigated 1180 by a factor of $\gamma_{01}$.

1181 Superimposed on the signal component $S^{0}[k] \cdot \mathbf{C}_{0}$, an 1182 AWGN vector $\mathbf{n}[k]$ consisting of $Q$ chip-related AWGN 1183 samples $n[(k-1) Q+q]$ is also received at $\mathrm{RN} r_{0}$ during 1184 "Phase 0", which may be formulated as [75]: $\mathbf{n}[k]=[n[(k-$ $11851) Q+1], n[(k-1) Q+2], \cdots, n[(k-1) Q+Q]]$, where we 1186 have $n[(k-1) Q+q] \sim \mathcal{C N}\left(0, \sigma^{2} / Q\right)$. Then, along with de1187 spreading the signal component at the DN $d$ by $\mathbf{C}_{1}^{T}$ at "Phase 11881 ", the AWGN vector $\mathbf{n}[k]$ is also despread. However, the 1189 corresponding term of $\mathbf{n}[k]$ is given by

$$
\eta_{1}=\sum_{q_{1}=1}^{Q} n\left[(k-1) Q+q_{1}\right] c_{1}\left[q_{1}\right] \sim \mathcal{C N}\left(0, \sigma^{2}\right),
$$

1190 where $\eta_{1}$ is a Gaussian variable, which still has a variance of $\sigma^{2}$. 1191 This implies that the power of the AWGN vector $\mathbf{n}[k]$ cannot 1192 be reduced by a single combined spread-despread operation. 1193 However, if $\eta_{1}$ is further spread by $\mathbf{C}_{0}$ and then despread by $1194 \mathbf{C}_{1}^{T}$, we obtain

$$
\eta_{2}=\sum_{q_{2}=1}^{Q} \eta_{1} c_{0}\left[q_{2}\right] c_{1}\left[q_{2}\right]
$$

1195 Observe that $\eta_{1}$ of (15) is a Gaussian random variable, but each 1196 of its realizations becomes a specific value in (16). Hence (16) 1197 may be rewritten as

$$
\eta_{2}=\eta_{1} \sum_{q_{2}=1}^{Q} c_{0}\left[q_{2}\right] c_{1}\left[q_{2}\right]=\eta_{1} \gamma_{01}
$$

1198 This implies that although the power of the AWGN vector $1199 \mathbf{n}[k]$ cannot be reduced by a single combined spread-despread 1200 operation, it can definitely be mitigated by multiple spread1201 despread operations.

1202 Inspired by the above results, a specifically arranged DS1203 CDMA spread-despread regime is designed in [82], which is 1204 portrayed in Fig. 24 for the sake of circumventing the potential 1205 noise accumulation process, and to mitigate both the successive 1206 relaying induced interferences and the MAI imposed on the

\footnotetext{
${ }^{8}$ It can be proved that $\left(1-f_{A M_{r_{i}}}^{2} G_{r_{\bar{i}} r_{i}}\right)$ is always less than 1 . Hence we have the relationship that $\sigma^{2} f_{A M_{r_{i}}}^{2} G_{r_{i} d} \cdot\left(1 / 1-f_{A M_{r_{i}}}^{2} G_{r_{\bar{i}} r_{i}}\right)>$ $\sigma^{2} f_{A M_{r_{i}}}^{2} G_{r_{i} d}$. Accordingly, the accumulated noise further aggravates the amplified and faded noise problem on the DN incurred in Section III-A.
}

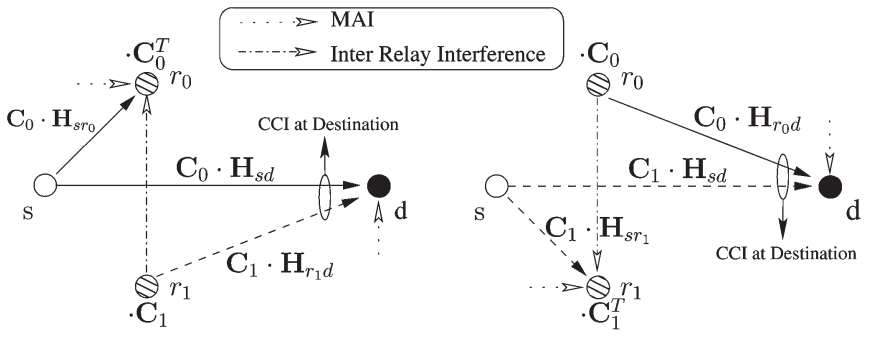

(a)

(b)

Fig. 24. The generalized successive relaying aided transmission processes and the specific spreading-despreading regime, when considering both the successive relaying induced interferences and the MAI. (a) Even Phase " $l \in\{0,2,4 \cdots\}$ ". (b) Odd Phase " $l \in\{1,3,5 \cdots\} "$ ".

cooperative DS-CDMA uplink. This specifically arranged DS- 1207 CDMA spreading-despreading regime is detailed as below: 1208

1) At the $\mathrm{SN} s$, the modulated symbols $S^{l}[k]$ are alternately 1210 spread by $\mathbf{C}_{0}$ and $\mathbf{C}_{1}$ from frame to frame $(l=0,1, \cdots) .1211$ For example, observe in Fig. 24 that the signals broadcast 1212 by the SN $s$ during the even phases of " $l=0,2,4, \cdots$ " 1213 are always spread by the PN sequence $\mathbf{C}_{0}$. By contrast, 1214 the signals are still broadcast by the $\mathrm{SN} s$, but during the 1215 odd phases of " $l=1,3,5, \cdots$ ", they are always spread 1216 by the other PN sequence $\mathbf{C}_{1}$.

2) At the $\mathrm{RN} r_{i}$, the received signals are firstly despread 1218 by $\mathbf{C}_{i}^{T}$ in its listening mode, and then they are directly 1219 spread by $\mathbf{C}_{i}$ in the transmit mode of the $\mathrm{RN} r_{i}$ before 1220 the amplification operation. For example, observe on the 1221 left hand side of Fig. 24 that the signal received at the 1222 $\mathrm{RN} r_{0}$ during an even phase, say "Phase 2" is despread 1223 by the PN sequence $\mathbf{C}_{0}^{T}$. As discussed below (16), after 1224 this despreading operation, the noise component imposed 1225 on the RN $r_{0}$ during "Phase 2" becomes a specific value. 1226 Then, the entire signal, which was received and further 1227 despread at the $\mathrm{RN} r_{0}$ during "Phase 2"-including 1228 the noise component-is spread by $\mathbf{C}_{0}$ and then for- 1229 warded to the RN $r_{1}$ during the consecutive odd phase 1230 "Phase 3", as shown in the right hand side of Fig. 24. 1231 Hence this noise component inherent in the interfering 1232 signal transmitted from the $\mathrm{RN} r_{0}$ to the $\mathrm{RN} r_{1}$ will be 1233 simultaneously suppressed at the RN $r_{1}$ by the despread- 1234 ing operation of $\cdot \mathbf{C}_{1}^{T}$ along with the entire interfering 1235 signal.

3) Hence, when we employ an appropriate PN sequence for 1237 suppressing the interfering signal transmitted by the RNs 1238 (e.g. the IRI) at the receiver (RN or DN), according to 1239 (17) the AF noise component inherent in the interference 1240 will be simultaneously suppressed. This implies that the 1241 noise accumulation process is indeed avoided.

4) This spreading-despreading scheme also guarantees that 1243 the two different components of the signal received at 1244 the DN, namely those which correspond to the SN's 1245 transmitted signal and to the RN's forwarded signal, re- 1246 spectively, are always spread by different PN sequences. 1247 This is evidenced by observing the pair of signal streams 1248 received at the DN $d$ in Fig. 24. 


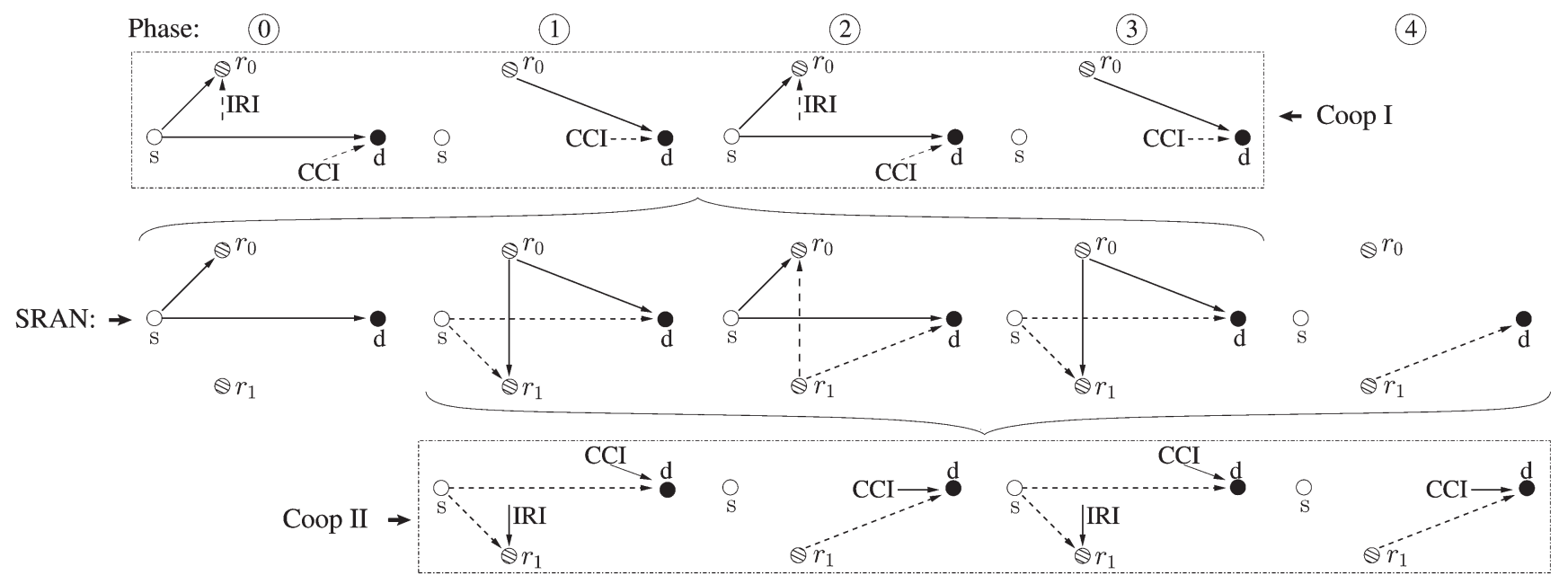

Fig. 25. Decomposition of the AF based SRAN.

1250 3) Analysis of the Signals Obtained at the DN: Based 1251 on the specific interference suppression regime introduced in 1252 Section III-C2, we can readily proceed to the analysis of the 1253 specific signal components received at the DN. However, for the 1254 sake of avoiding complicated mathematical manipulations, in 1255 this tutorial paper we refer to [81, Section II.D-E], especially to 1256 [81, Eq.(15-16)] for further details of these signal components.

\section{D. Successive AF Relaying Aided Multi-User DS-CDMA 1258 Uplink: NC DCMC Capacity}

1259 In Section III-B, we focussed our attention on the BER 1260 performance versus complexity of the prototype system of 1261 Fig. 17 introduced in Section III-A. Based on [82], in this 1262 section, we would like to extend our discussions to the theo1263 retical capacity of the prototype system of Fig. 22 introduced in 1264 Section III-C. In more detail, based on the idealized simplifying 1265 assumption that the CSI is perfectly known, the capacity of 1266 the AF based cooperative systems was characterized in [83] 1267 and [84]. However, the NC DCMC capacity of the AF based 1268 SRAN is still unknown. Hence we focus our attention on the 1269 NC DCMC capacity of the successive AF relaying aided multi1270 user DS-CDMA system herein for characterising its theoretical 1271 bound.

1272 The critical issue behind the capacity derivation of the AF 1273 based SRAN is that the transmission arrangement of the twin1274 relay-aided successive relaying procedure of Fig. 24 may be 1275 viewed as the superimposed transmissions of two conventional 1276 single-relay aided two-phase cooperative links [85]. This has 1277 been illustrated in Fig. 24, where the transmissions represented 1278 by the solid lines in the even phase and odd phase constitute 1279 one of the half-duplex three-terminal cooperative networks, 1280 namely Coop-I. Similarly, the transmissions represented by the 1281 dashed lines in the even and odd phases constitute another one, 1282 namely Coop-II.

1283 To further clarify this decomposition of the AF based SRAN, 1284 we illustrate its detailed transmission process in Fig. 25, where 1285 the transmissions of the SRAN were split into five phases. 1286 Observe in Fig. 25 that the transmission arrangement of the five-phase SRAN may be treated as the superposition of the 1287 transmissions of a pair of four-phase based half-duplex three- 1288 terminal cooperative networks, which are the above mentioned 1289 sub-networks "Coop-I" and "Coop-II". The slight difference 1290 between our sub-network "Coop-I" (or "Coop-II") and the 1291 conventional half-duplex three-terminal cooperative network is 1292 that we additionally imposed the omni-present CCI and IRI on 1293 the DN and RN of the sub-networks, respectively, because we 1294 have to assume that an equivalent amount of CCI (or IRI) is also 1295 imposed on the DN of the sub-networks, just like that which 1296 happens to the AF based SRAN located in the middle of Fig. 25. 1297

Hence, we can readily extend the relationship between the 1298 AF based SRAN and the pair of half-duplex three-terminal 1299 cooperative networks based on Fig. 25 to the generalized sce- 1300 nario, where the transmission arrangement of an $(N+1)$-phase 1301 SRAN may be treated as a superposition of the transmissions of 1302 two $N$-phase half-duplex three-terminal cooperative networks. ${ }^{9} 1303$ Hence, assuming that $(N+1)$ is sufficiently high, we may 1304 readily conclude that the noncoherent DCMC capacity of the 1305 AF based SRAN is constituted by the sum of the capacities of 1306 the AF based sub-network "Coop-I" and "Coop-II", i.e.

$$
C_{\text {Successive }}^{\mathrm{AF}}=C_{\mathrm{Coop}-\mathrm{I}}^{\mathrm{AF}}+C_{\mathrm{Coop}-\mathrm{II}}^{\mathrm{AF}} .
$$

Consequently, the problem is transformed to that of deriving 1308 the NC DCMC capacity of the AF based half-duplex three- 1309 terminal cooperative network e.g. $C_{\mathrm{Coop}-\mathrm{I}}^{\mathrm{AF}}$. From a capacity 1310 analysis perspective, the sub-network Coop-I may be equiva- 1311 lently modelled by a single-input-multiple-output (SIMO) sys- 1312 tem having $N_{T}=1$ transmit and $N_{R}=2$ receive antennas. 1313 The evaluation of the NC DCMC capacity of the equivalent 1314 SIMO system may be carried out according to the principles 1315 introduced in [84]-[86]. Hence, the evaluation of $C_{\text {Successive }}^{\mathrm{AF}}$ in 1316 (18) becomes feasible.

\footnotetext{
${ }^{9}$ The transmission arrangements of the first and last phases in the AF based SRAN do not strictly satisfy our decomposition of the AF based SRAN. Nevertheless, when the total number of transmission phases in the AF based SRAN is sufficiently large, we may ignore the slight inaccuracy incurred in the two particular phases.
} 
TABLE X

Distance Ratios CONSIDERED

\begin{tabular}{|c|cccc|}
\hline$\theta$ & $D_{s r_{i}}$ & $D_{r_{i} d}$ & $D_{s d}$ & $D_{r_{0} r_{1}}$ \\
\hline $1 / 3$ & 1 & 3 & 3.824 & 1 \\
\hline $1 / 2$ & 1 & 2 & 2.802 & 1 \\
\hline 1.0 & 1 & 1 & 1.732 & 1 \\
\hline 2.0 & 2 & 1 & 2.802 & 1 \\
\hline 3.0 & 3 & 1 & 3.824 & 1 \\
\hline
\end{tabular}

TABLE XI

SYSTEM PARAMETERS

\begin{tabular}{|l|r|}
\hline Scenario & Multi-User DS-CDMA Uplink \\
\hline System Regime & Successive AF Relaying \\
\hline Channel Model & Block Fading Channel [87] \\
\hline Correlated Fading Block Length & $T_{b}=6$ \\
\hline Normalized Doppler Frequency & $f_{d}=0.01$ \\
\hline PN sequence & $Q=127$ \\
\hline Spreading Factor & Differential QPSK \\
\hline Modulation Scheme & $P_{s}=P_{r_{i}}=\frac{1}{2} P_{\text {total }}$ \\
\hline Power Allocation & $M \in\left\{\frac{1}{3}, \frac{1}{2}, 1.0,2.0,3.0\right\}$ \\
\hline RN's Position & $M \in\{0,32,64\}$ \\
\hline Number of Interfering Users & $\lambda=2.0$ \\
\hline Ratio of RN's MAI to DN's MAI & \\
\hline
\end{tabular}

1318 In the remaining discussions of this section, we would like 1319 to quantify $C_{\text {Successive }}^{\mathrm{AF}}$ with the aid of the associated formulas 1320 provided in [81]. Firstly, let us define the distance-ratio of $1321 \theta=\left(D_{s r_{i}} / D_{r_{i} d}\right)$, and assume that $D_{r_{0} r_{1}}$ equals a relatively 1322 small value between $D_{s r_{i}}$ and $D_{r_{i} d}$. Based on the symmetrical 1323 structure of the proposed SRAN assumed in Section III-C, the 1324 exact proportions of $D_{s r_{i}}, D_{r_{i} d}, D_{s d}$ and $D_{r_{0} r_{1}}$ associated 1325 with different $\theta$ values are summarized in Table X, where the 1326 shortest distance is always normalized to unity. Then, let $\lambda$ 1327 represent the ratio of the MAI imposed on the RN $r_{i}$ to the MAI 1328 imposed on the BS. Naturally, the actual value of $\lambda$ will vary, 1329 depending on the specific network topology. As a reasonable 1330 value, $\lambda=2.0$ is assigned in our simulations. Then, the power 1331 allocation strategy, the channel model, the correlated block1332 fading period of $T_{b}$, as well as all other system parameters 1333 utilized in our forthcoming simulations of this section are 1334 summarized in Table XI.

1335 When a cooperative-user-selection scheme is employed, the 1336 effect of the RN's position is displayed in Fig. 26, where 1337 the RN positions expressed in terms of $\theta \in\{3.0,2.0,1.0$, $1338(1 / 2),(1 / 3)\}$ were considered and the number of interfering 1339 users was fixed to $M=0$. Observe in Fig. 26 that the capacity 1340 of the SN's uplink employing the AF based SRAN exceeds 1341 that of the conventional SL DT structure, when assigning RNs 1342 sufficiently close to the SN. By contrast, it results in a degraded 1343 capacity compared to the conventional direct transmission 1344 structure, when assigning RNs close to the DN. In our particular 1345 case, assigning the RNs at the position $\theta=(1 / 2)$ is seen to be 1346 the best strategy in Fig. 26, which slightly improves the capacity $1347 C_{\text {Successive }}^{\mathrm{AF}}$ compared to the scenarios $\theta=(1 / 3)$ and $\theta=1.0$. 1348 The capacity of the conventional single-relay aided two-phase 1349 AF based cooperative DS-CDMA uplink having $\theta=(1 / 2)$ is 1350 also shown in Fig. 26, which is significantly exceeded by that 1351 of its AF based SRAN counterpart.

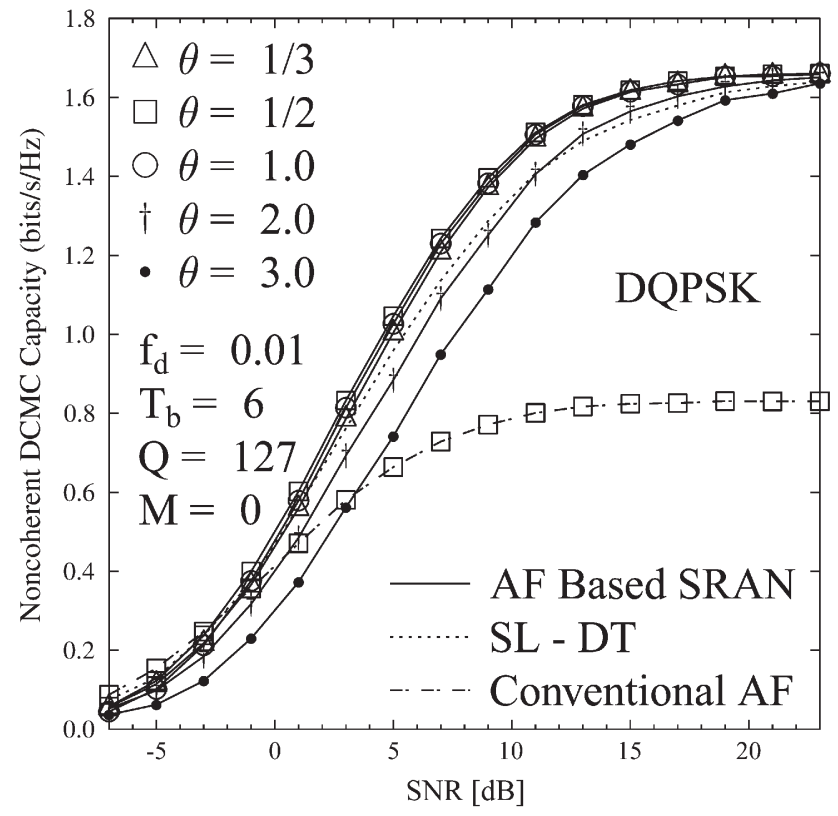

Fig. 26. The effect of the geographic position of the $\mathrm{RN}$ on $C_{\mathrm{Successive}}^{\mathrm{AF}}$.

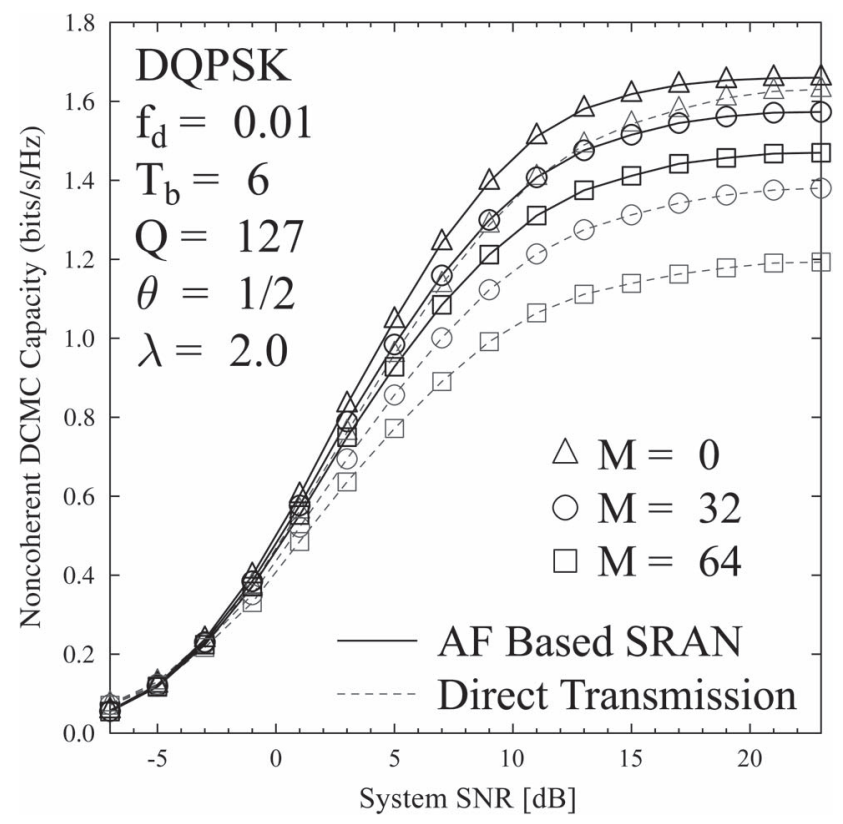

Fig. 27. The noncoherent DCMC capacity $C_{\text {Successive }}^{\mathrm{AF}}$ in zero, moderate and heavy MAI scenarios.

In our next investigation we fix the position of the RNs at $\theta=1352$ $(1 / 2)$ and focus our attention on the detrimental effects of hav- 1353 ing an increased number of interfering users $M$. Specifically, 1354 the values of $M \in\{0,32,64\}$ are considered for modelling the 1355 scenarios of zero, moderate and heavy MAI, respectively. The 1356 relevant simulation results displayed in Fig. 27 demonstrate 1357 that the capacity of the AF based SRAN always exceeds that 1358 of the direct transmission system, regardless of the number 1359 of interfering users. It is more intriguing to find that the 1360 capacity advantage of the AF based SRAN with respect to 1361 the conventional direct transmission system increases, when 1362 the MAI becomes stronger. This implies that in contrast to the 1363 
1364 conventional DS-CDMA uplink, the cooperative DS-CDMA 1365 uplink will exhibit more substantial advantages in high-load 1366 situations.

1367

1368

1369 Based on the interference suppression regime introduced in 1370 Section III-A as well as in Section III-C2, it is feasible to ef1371 ficiently suppress the successive relaying induced interference 1372 with the aid of the classic DS-CDMA multiple access tech1373 nique. Hence, we are capable of operating the SR aided system 1374 in a week IRI scenario. Then, according to the analysis provided 1375 in Section II-A, especially to the comparisons demonstrated in 1376 Fig. 9, we may replace the AF protocol employed in Section III 1377 by the DF protocol for the sake of achieving a potentially 1378 better BER performance. Therefore, a successive DF relaying 1379 aided multi-user DS-CDMA uplink architecture is conceived. 1380 Then, the SDB relay-aided MSDSD algorithm introduced in 1381 Section II-E can be employed in this system for realizing 1382 energy-efficient detection. The resulting DF based SRAN leads 1383 to a sophisticated relay-aided SISO-MSDSD assisted three1384 stage iterative-detection-based transceiver architecture, which 1385 will be detailed in Section IV-C.

\section{A. System Model of DF Based SRAN}

1387 The network's topology illustrated in Fig. 22 is still valid 1388 for the DF based SRAN. Consequently, both the MAI and 1389 the successive relaying induced interferences, namely the CCI 1390 and IRI contributions are also taken into account in the DF 1391 based system model. Furthermore, the relaying-related path1392 loss reduction effects and the power-allocation as well as the 1393 channel model are also assumed to be the same as those defined 1394 in Section III-C. All the notation given in Section III will retain 1395 the original definitions in this section.

1396 The DS-CDMA based interference suppression regime intro1397 duced in Section III-C2 is also valid for the DF based SRAN. 1398 In more detail, at the $\mathrm{SN} s$, the modulated symbols $S^{l}[k]$ are 1399 alternately spread by the spreading sequences of $\mathbf{C}_{0}$ and $\mathbf{C}_{1}$ 1400 from frame to frame $(l=0,1, \ldots)$. At the $\mathrm{RN} r_{i}$, the received 1401 signals are firstly despread by $\mathbf{C}_{i}^{T}$ in the listening mode of RN $1402 r_{i}$ and then decoded, re-encoded, as well as re-modulated. In the 1403 consecutive transmit mode of $\mathrm{RN} r_{i}$, the re-modulated symbols $1404 \tilde{S}^{l}[k]$ are always spread by $\mathbf{C}_{i}$, which are then forwarded to 1405 the DN. Again, this spreading scheme is the same to that 1406 depicted in Fig. 24, where only the decoding, re-encoding and 1407 re-modulating process is omitted. Thus, the spreading scheme 1408 also guarantees that the two different components of the signal 1409 received at the DN, namely those that correspond to the SN's 1410 transmitted signal and to the RN's forwarded signal, respec1411 tively, are always spread by different PN sequences.

1412 When employing the DF protocol, the despread signal of 1413 the $(l-1)^{s t}$ frame at RN $r_{i}$ associated with $S^{l-1}[k]$ is rep1414 resented by $y_{r_{i}}^{l-1}[k]$. At the $\mathrm{DN}$, the received signal is repre1415 sented by $\mathbf{y}^{l}[k]$. Hence, similar to the mechanism described in 1416 Section III-C3, when the despreading matched-filter is applied 1417 to the waveform of $\mathbf{C}_{\bar{i}}$, the signal directly transmitted by the SN will contribute the main component of the despread 1418 signal, while the RN's forwarded signal and the interfering 1419 user's signals become the interference. The resulting output 1420 of the matched-filter may be represented by $z_{s}^{l}[k]$, which cor- 1421 responds to the information bearing symbol $S^{l}[k]$. Similar to 1422 Section III-A, let us define $\mathbf{Z}_{s}^{l}=\left[z_{s}^{l}[1], z_{s}^{l}[2], \ldots, z_{s}^{l}[N]\right]^{T}, 1423$ which corresponds to the symbol vector $\mathbf{S}^{l}=\left[S^{l}[1], S^{l}[2], 1424\right.$ $\left.\ldots, S^{l}[N]\right]^{T}$ broadcast by the SN during the $l^{t h}$ frame. 1425

Similarly, when the filter is matched to the waveform $\mathbf{C}_{i}, 1426$ another despread signal can also be extracted from the received 1427 signal $\mathbf{y}^{l}[k]$, which is dominated by the RN's re-modulated 1428 symbol $^{10} S^{l}[k]$ and may be represented by $z_{r_{i}}^{l}[k]$. If we 1429 observe the consecutive $(l+1)$ st frame, we obtain $\mathbf{Z}_{r_{\bar{i}}}^{l+1}=1430$ $\left[z_{r_{\bar{i}}}^{l+1}[1], z_{r_{\bar{i}}}^{l+1}[1], \ldots, z_{r_{\bar{i}}}^{l+1}[N]\right]^{T}$, which also corresponds to the 1431 symbol vector of $\mathbf{S}^{l}$. Hence, according to the principles intro- 1432 duced in Section II-E, we can now proceed by implementing 1433 the SDB relay-aided MSDSD algorithm at the DN by utilizing 1434 $\mathbf{Z}_{s}^{l}$ and $\mathbf{Z}_{r_{\bar{i}}}^{l+1}$ as the $\mathbf{z}_{u}$ components in (10).

\section{B. NC DCMC Capacity}

As stated at the beginning of this section, the motivation 1437 for replacing the AF protocol by the DF protocol is that of 1438 achieving a potentially better BER performance. Hence the NC 1439 DCMC capacity of the DF based SRAN will be considered in 1440 the context of the multi-user DS-CDMA uplink.

The decomposition of the SRAN illustrated in Fig. 25 remains 1442 valid for the DF based system model depicted in Section IV-A. 1443 Hence, in the spirit of Section III-D, the NC DCMC capacity 1444 of the DF based SRAN is also constituted by the sum of the 1445 capacities of the pair of sub-networks Coop-I and Coop-II, 1446 which is formulated as

$$
C_{\text {Successive }}^{\mathrm{DF}}=C_{\mathrm{Coop}-\mathrm{I}}^{\mathrm{DF}}+C_{\mathrm{Coop}-\mathrm{II}}^{\mathrm{DF}},
$$

where the sub-networks Coop-I and Coop-II illustrated in 1448 Fig. 25 of Section III-D now rely on the DF protocol. Then the 1449 NC DCMC capacity of these sub-networks can be evaluated 1450 according to the principles introduced in [16], [85], [88], and 1451 [81]. Hence the evaluation of $C_{\text {Successive }}^{\mathrm{DF}}$ in (19) becomes 1452 feasible. Moreover, to compare this DF SRAN capacity to the 1453 capacity of the AF based system characterised in Section III-D, 1454 the parameters related to the NC DCMC capacity in this section 1455 are the same as those specified in Table XI.

1456

The attainable capacity $C_{\text {Successive }}^{\mathrm{DF}}$ associated with different 1457 RN positions is shown in Fig. 28. This demonstrates that 1458 assigning RNs that roam closer to the $\mathrm{SN}$-rather than to the 1459 DN-in the DF based SRAN provides a higher capacity. To 1460 expound a little further, in the low SNR region $(<1 \mathrm{~dB})$, the DF 1461 based system assigning RNs at the position $\theta=1.0$ achieves 1462 the highest capacity. The phenomenon noted in Section III-D, 1463 namely that the capacity gain achieved by replacing the direct 1464

\footnotetext{
${ }^{10}$ The $\mathrm{RN}$ receives the information-bearing symbol $S^{l-1}[k]$ during $(l-$ $1)^{s t}$ frame. After decoding and re-encoding, the re-modulated symbol $\tilde{S}^{l}[k]$ is forwarded from the RN to the DN during the $l^{\text {th }}$ frame.
} 


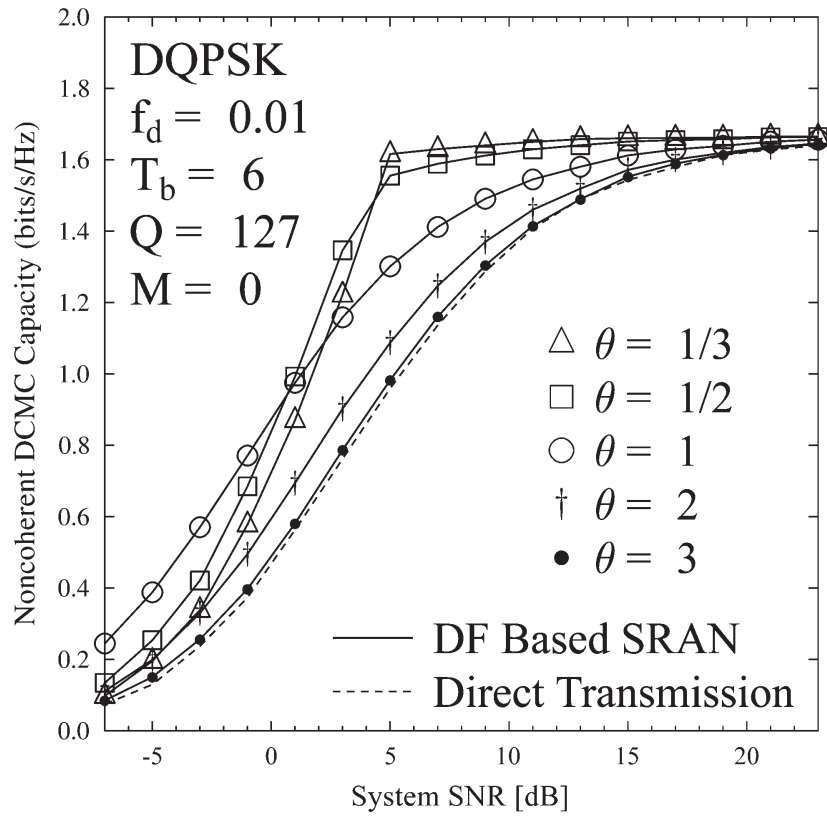

Fig. 28. The effect of the geographic position of the $\mathrm{RN}$ on $C_{\mathrm{Successive}}^{\mathrm{DF}}$, as evaluated from (19).

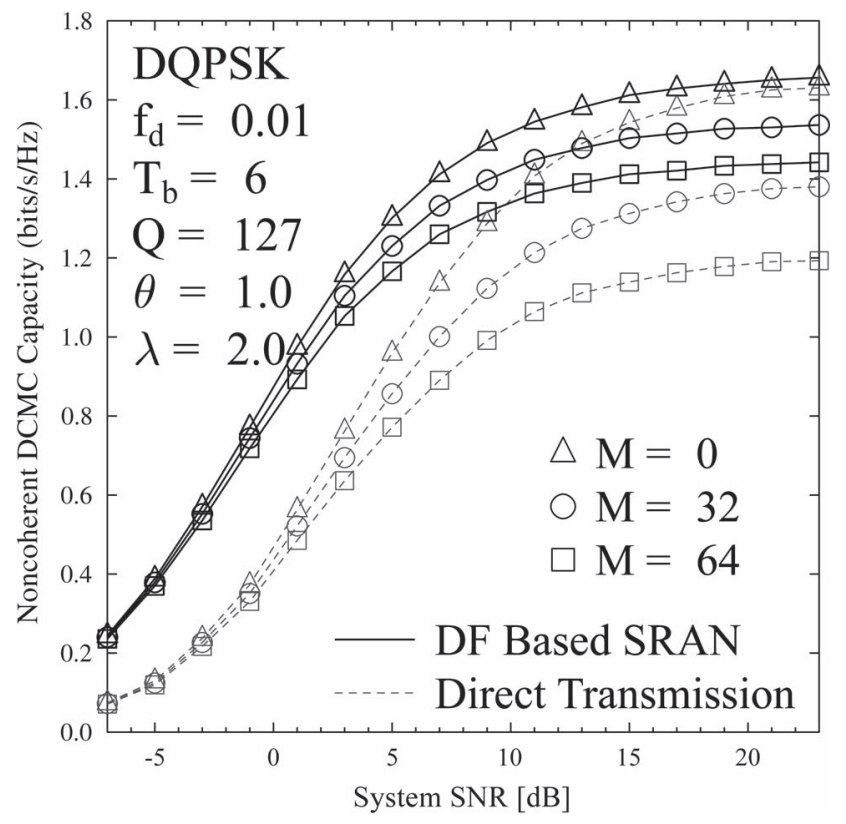

Fig. 29. The noncoherent DCMC capacity $C_{\mathrm{Suc}}^{\mathrm{DF}}$ heavy MAI scenarios, as evaluated from (19).

1465 transmission structure with the SRAN increases upon increas1466 ing the number of interfering users remains valid, when con1467 sidering the DF based SRAN, as demonstrated by Fig. 29. 1468 Furthermore, upon comparing Fig. 26 to Fig. 28, we observe 1469 that the DF based SRAN assigning appropriate RNs outper1470 forms AF based SRAN, especially at low SNRs, i.e. in the 1471 low-throughput region. Hence our objective introduced at the 1472 beginning of this section, namely that of improving the system's 1473 energy efficiency and/or spectral efficiency by replacing the AF 1474 protocol by the DF protocol is indeed achievable.

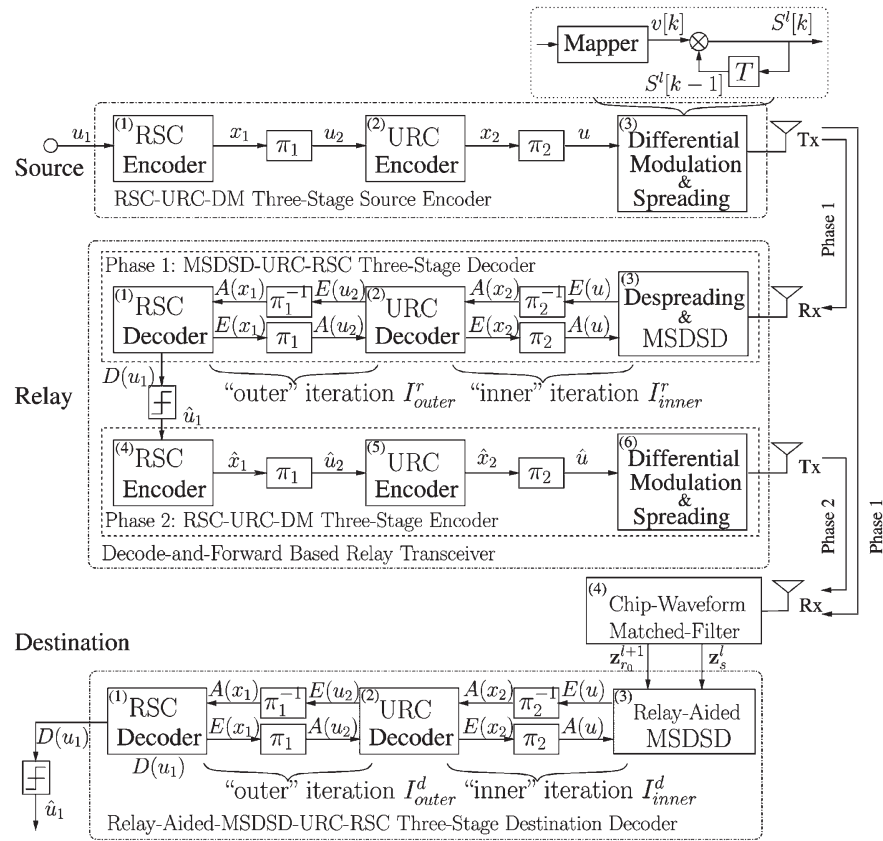

Fig. 30. Schematic of the proposed transceiver in the DF based SRAN.

\section{Three-Stage Iterative Decoder Design}

Based on the relay-aided SISO-MSDSD algorithm addressed 1476 in Section II-E, in this section, we design a three-stage iterative 1477 detection based transceiver architecture. The complexity of the 1478 proposed relay-aided SISO-MSDSD algorithm is characterized 1479 at the end of this section.

1480

The transceiver architecture specifically designed for the 1481 DF based SRAN is portrayed in Fig. 30. At the SN, we use 1482 a conventional differential modulator (DM), such as DQPSK 1483 depicted at the top right corner of Fig. 30, which is further 1484 combined with a unity-rate-code (URC) encoder to create a 1485 two-stage inner code. The URC model has an infinite impulse 1486 response due to its recursive encoder structure, consequently 1487 the extrinsic information transfer (EXIT) curve [89] of the URC 1488 aided inner decoder is capable of approaching the point of per- 1489 fect convergence at $(1.0,1.0)$ in the EXIT chart, which is a nec- 1490 essary condition for near-capacity operation [8], [71], and for 1491 eliminating the potential error floor phenomenon. Therefore, 1492 the receiver of the RN is capable of near-perfectly detecting the 1493 information bits $\hat{u}_{1}$ borne in the signals received from the SN, 1494 at as low SNR values as possible. Furthermore, a conventional 1495 half-rate recursive systematic convolutional (RSC) code is em- 1496 ployed as the outer code. Hence a three-stage RSC-URC-DM 1497 source encoder is created.

The corresponding URC decoder assisted three-stage re- 1499 ceiver proposed for the relay is also portrayed in Fig. 30. 1500 In more detail, the RN's receiver consists of three stages, 1501 namely the conventional single-path SISO-MSDSD [66] based 1502 soft decoder, the URC decoder and the RSC decoder. The 1503 extrinsic information and a priori information, represented 1504 by $E(\cdot)$ and $A(\cdot)$ respectively, are interleaved and iteratively 1505 exchanged within the two-stage inner decoder $I_{\text {inner }}^{r}$ times, 1506 before the result is further exchanged between the inner and 1507 outer decoders $I_{\text {outer }}^{r}$ times. The motivation for employing this 1508 


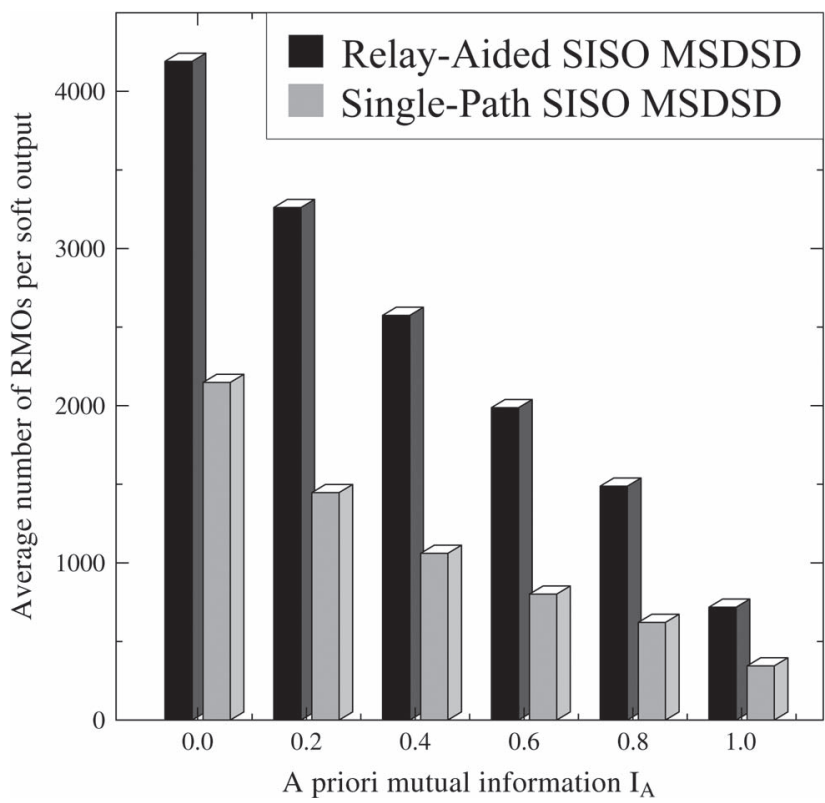

Fig. 31. Complexity comparison between the SDB relay-aided MSDSD decoder and the conventional single-path SISO-MSDSD decoder at different a priori mutual information values.

1509 three-stage concatenated decoder architecture is to improve the 1510 convergence behavior of the iterative decoder with the aid of 1511 the URC decoder, as detailed in [8] and [71]. As a benefit, 1512 the error propagation problem of the DF scheme is avoided. 1513 Since the proposed SISO-MSDSD decoder will be employed 1514 at the $\mathrm{DN}$, we have to ensure that its multiple input signal 1515 streams are corresponding to the same differentially modulated 1516 symbols. The RN's transmitter is designed to be identical to the 1517 three-stage RSC-URC-DM encoder of the SN. Hence, observe 1518 at the RN in Fig. 30 that if the estimates $\hat{u}_{1}$ are correctly 1519 generated by the RN's receiver, the differentially modulated 1520 symbols produced by the RN's transmitter will be the same 1521 as $S^{l}[k]$.

1522 As seen in Fig. 30, the resulting relay-aided SISO-MSDSD 1523 decoder is employed as the first stage of the iterative receiver 1524 at the DN, which is then further combined with the URC 1525 decoder to form a two-stage inner decoder for appropriately 1526 complementing the SN's and RN's transmitter architecture. 1527 Then, a RSC decoder is concatenated with the relay-aided1528 SISO-MSDSD-URC two-stage inner decoder for creating the 1529 DN's three-stage decoder seen in Fig. 30.

1530 Our complexity comparison between the single-path SISO1531 MSDSD decoder advocated in [66] and the SDB relay-aided 1532 MSDSD decoder is provided in Fig. 31. In the spirit of [66], 1533 the average number of real-valued multiplication operations 1534 (RMOs) required for generating a single soft-output during the 1535 SDB MSDSD detection once per iteration is employed here 1536 as the complexity measure. For the sake of a fair compari1537 son, we ensured that both the conventional single-path SISO1538 MSDSD decoder and the SDB relay-aided MSDSD decoder 1539 were operated near their associated "turbo-cliff" points. Then 1540 we varied the a priori mutual information of the two different 1541 SDB MSDSD decoders and recorded the associated number 1542 of RMOs required for producing a single soft-output once per iteration. Observe in Fig. 31 that the SDB relay-aided MSDSD 1543 decoder approximately doubles the complexity compared to 1544 the single-path SISO-MSDSD decoder, which is valid right 1545 across the entire a priori mutual information region considered. 1546 The remaining components of the DN's receiver are similar to 1547 those of the RN, hence they affect the overall complexity in a 1548 similar way.

When designing an iterative decoding aided cooperative sys- 1550 tem, the distributed turbo coding scheme advocated in [90] and 1551 [91] is attractive, since it benefits from the iterative information 1552 exchange between the direct and relayed versions of the same 1553 codeword, which experience uncorrelated fading. Naturally, 1554 the improved system performance is achieved at the cost of 1555 increasing the complexity imposed by employing an extra 1556 iteration stage.

By contrast, the relay-aided SISO-MSDSD algorithm consti- 1558 tutes a realistic method of maintaining low complexity, where 1559 the combination of the information provided by the direct and 1560 relayed signal streams, namely by $\mathbf{z}_{s}^{l}$ and $\mathbf{z}_{r_{0}}^{l+1}$ is achieved 1561 without an extra iteration stage. More explicitly, in a cooper- 1562 ative network, where the distributed turbo coding principle is 1563 employed by invoking the single-path SISO-MSDSD [66] al- 1564 gorithm, as in [88], each input signal stream is first individually 1565 processed by a single-path SISO-MSDSD aided turbo decoder 1566 within the inner iterative stage of [88, (Figure 7)]. Then the 1567 resulting information is passed on to the outer iterative stage, 1568 and typically at least two iterations are carried out to exchange 1569 information between the different input signal streams. Hence, 1570 based on our complexity comparisons shown in Fig. 31, it 1571 is reasonable to argue that the three-stage relay-aided-SISO- 1572 MSDSD-URC-RSC decoder is capable of halving the system 1573 complexity imposed by the conventional single-path SISO- 1574 MSDSD aided distributed turbo decoder.

\section{Transceiver Performance: Robustness, Throughput and Complexity}

In this subsection, we firstly characterise the robustness of 1578 the three-stage relay-aided-SISO-MSDSD-URC-RSC decoder 1579 depicted in Fig. 30 in the terms of its BER performance. We 1580 commence by identifying the "turbo-cliff" SNR with the aid 1581 of EXIT-charts as detailed in [8]. The relevant EXIT-chart and 1582 BER results of the DF based SRAN are shown in Figs. 32 and 1583 33, respectively, when using the system parameters summarized 1584 in Table XII.

Observe in Fig. 32 that an open tunnel exists between the 1586 EXIT curves of the two-stage inner relay-aided-SISO-MSDSD- 1587 URC decoder and the outer RSC decoder, when the overall 1588 equivalent SNR value ${ }^{11}$ reaches $2.12 \mathrm{~dB}$. Furthermore, the 1589 associated Monte-Carlo simulation based decoding trajectory 1590 closely matches the EXIT curves. Correspondingly, an in- 1591 finitesimally low BER is expected beyond the $\mathrm{SNR}=2.12 \mathrm{~dB} 1592$ point. This is further evidenced in Fig. 33. The capacity of the 1593

\footnotetext{
${ }^{11}$ Here the terminology of "equivalent SNR" is defined as the ratio of the transmit power to the receiver's noise, which are measured at physically different points.
} 


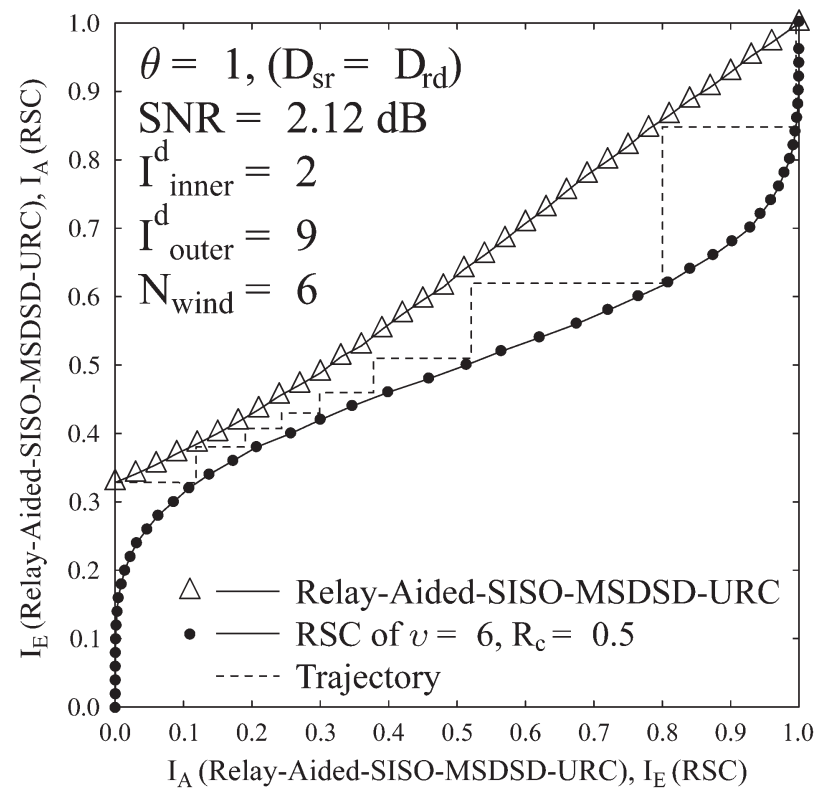

Fig. 32. The EXIT characteristic of the three-stage relay-aided-SISOMSDSD-URC-RSC destination receiver.

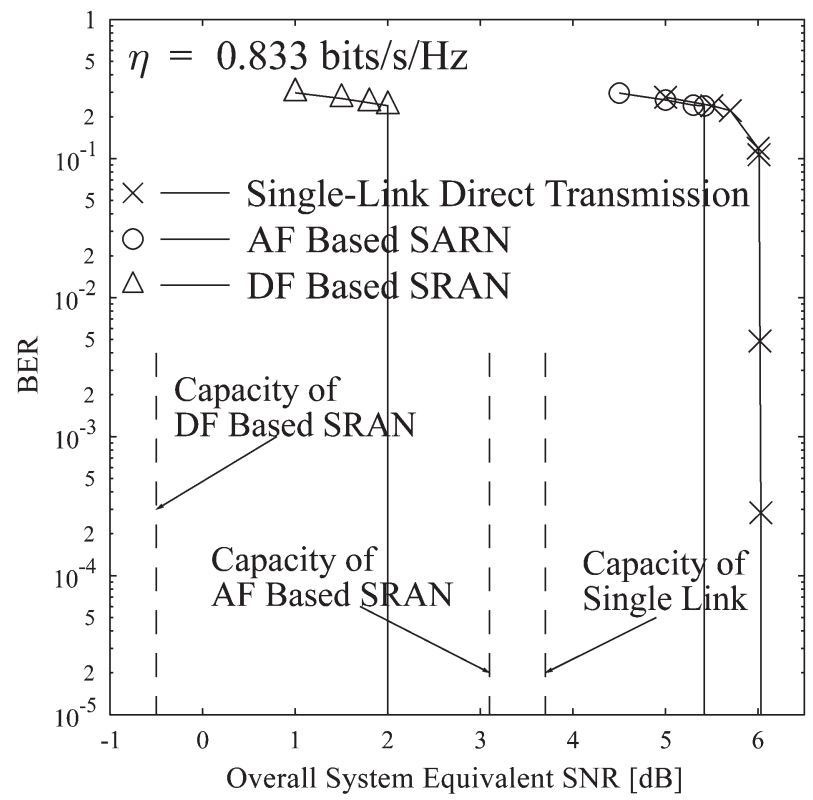

Fig. 33. A comparison of BER performance of different systems.

1594 proposed DF based SRAN is also characterized in Fig. 33, 1595 which can be directly obtained from Fig. 28. In our 1596 case, the corresponding spectral efficiency is $\eta \approx R_{c} \times$ $1597 \log _{2} M_{c} \times\left(T_{b}-1 / T_{b}\right)=0.8333 \mathrm{bit} / \mathrm{s} / \mathrm{Hz}$. Hence, the pro1598 posed transceiver architecture of Fig. 30 attains a performance 1599 within $2.6 \mathrm{~dB}$ of the capacity of the DF based SRAN.

1600 To elaborate further, observe in Fig. 33 that an approximately $16013.9 \mathrm{~dB}$ power reduction is achieved by the proposed DF scheme 1602 in comparison to the classic direct transmission regime. By 1603 contrast, its corresponding AF based counterpart attains a more 1604 modest power reduction of about $0.5 \mathrm{~dB}$, which is attained at a 1605 lower complexity than that of the DF arrangement.
TABLE XII

SYSTEM PARAMETERS

\begin{tabular}{|l|r|}
\hline Scenario & Multi-User DS-CDMA Uplink \\
\hline System Regime & Successive DF Relaying \\
\hline Channel Model & Block Fading Channel $[87]$ \\
\hline Path-Loss Exponent & $\alpha=3$ \\
\hline Correlated Fading Block Length & $T_{b}=6$ \\
\hline Normalized Doppler Frequency & $f_{d}=0.01$ \\
\hline PN Sequence & Gold Sequence \\
\hline Spreading Factor & $Q=127$ \\
\hline Channel Coding & RSC Code \\
\hline Code Memory Length & $\nu=6$ \\
\hline Code Rate & $R_{c}=0.5$ \\
\hline Interleaver Length & $4.8 \times 10^{5}$ Symbols \\
\hline Modulation Scheme & Differential QPSK \\
\hline MSDSD Window Size & $N_{\text {wind }}=6$ \\
\hline Power Allocation & $P_{s}=P_{r_{i}}=\frac{1}{2} P_{\text {total }}$ \\
\hline Inner Iterations of DN's Decoder & $I_{\text {inner }}^{d}=2$ \\
\hline Outer Iterations of DN's Decoder & $I_{\text {outer }}^{d}=9$ \\
\hline \multirow{2}{*}{ Relay Position in AF SRAN } & $\theta=\frac{1}{2}$, \\
\cline { 2 - 2 } & $G_{s r_{i}}=\left(\frac{1.0}{0.357}\right)^{3}, G_{r_{i} d}=\left(\frac{1.0}{0.714}\right)^{3}$ \\
\hline \multirow{2}{*}{ Relay Position in DF SRAN } & $\theta=1.0$, \\
\cline { 2 - 2 } & $G_{s r_{i}}=G_{r_{i} d}=\left(\frac{1.0}{0.577}\right)^{3}$ \\
\hline Overall Spectral Efficiency & $\eta=0.8333$ bits/s/Hz \\
\hline
\end{tabular}

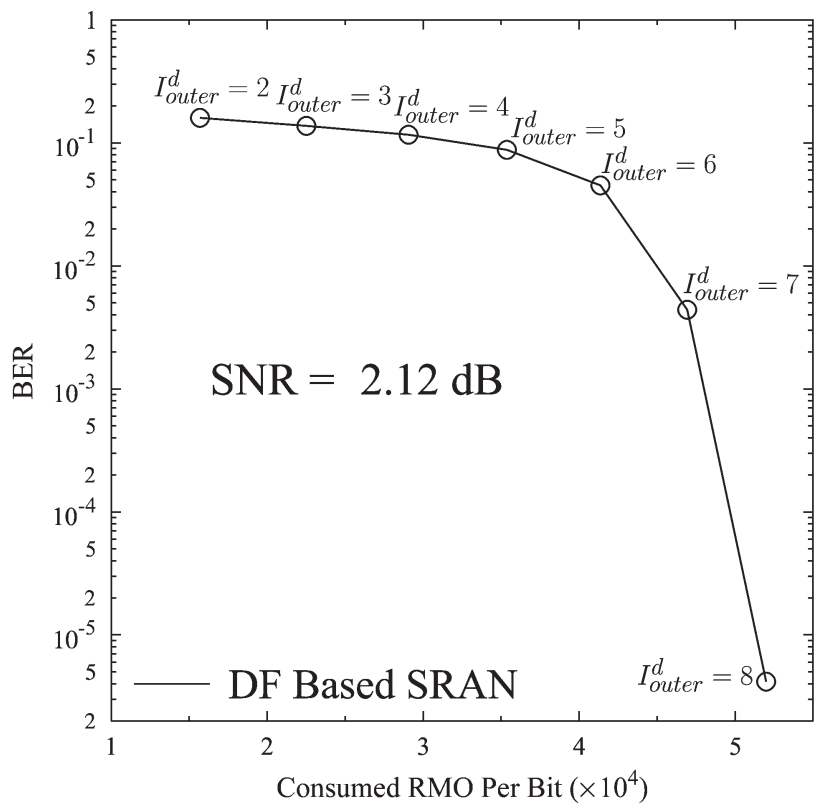

Fig. 34. The BER performance versus the complexity per bit, where the number of outer iterations of the DN's three-stage turbo decoder seen in Fig. 30 increases from $I_{\text {outer }}^{d}=2$ to $I_{\text {outer }}^{d}=8$, while the SNR value is fixed at $2.12 \mathrm{~dB}$. The results are based on the schematic of Fig. 30. The remaining parameters employed for generating the results are listed in Table XII.

Then, in Fig. 34, we quantitatively characterize the com- 1606 promise between the BER performance attained and the extra 1607 complexity imposed by the transceiver illustrated in Fig. 30. As 1608 expected, Fig. 34 demonstrates that the BER is improved by 1609 investing in increased computational complexity in terms of in- 1610 creasing the number of outer iterations. Furthermore, increasing 1611 $I_{\text {outer }}^{d}$ to 9 will result in an infinitesimally low BER, as shown 1612 in Fig. 32.

Based on the results shown in Fig. 20, it may be anticipated 1614 that the energy efficiency of the NC detection aided system 1615 architecture of Fig. 30 will be improved by increasing the 1616 observation window size of the MSDD algorithms employed. 1617 


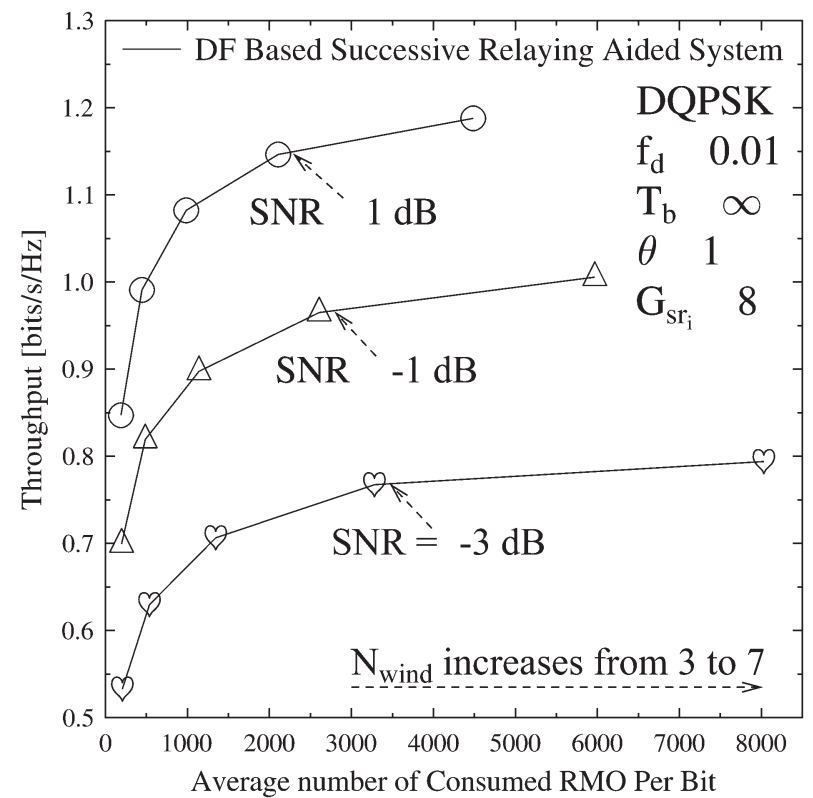

Fig. 35. The trade-off between the maximum achievable throughput and the complexity per bit, where various values of $N_{\text {wind }}$ are investigated.

1618 Equivalently, if we fix the transmit power, the system's spectral 1619 efficiency will also benefit from increasing the observation win1620 dow size. However, this improvement of the spectral efficiency 1621 is achieved at the cost of imposing an increased complexity on 1622 the system, especially on the MSDD aided decoder, as observed 1623 for example in Fig. 21. Hence we compare the system's com1624 plexity imposed by employing different observation window 1625 sizes $N_{\text {wind }}$ in the context of the transceiver architecture of 1626 Fig. 30. Then, it can be shown that only the complexity imposed 1627 by increasing the observation window size of the SDB relay1628 aided MSDSD decoder has to be involved in the complexity 1629 comparison, where the average number of RMOs required for 1630 producing a single soft-output once per iteration is used again 1631 as the complexity measure.

1632 Consequently, the trade-off between the maximum achiev1633 able throughput and the complexity imposed is visualized in 1634 Fig. 35, where different SNR values are considered. In Fig. 35, 1635 the path-loss gain of $G_{s r_{i}}$ is chosen to be 8 , which is different 1636 from that specified in Table XII. As demonstrated in Fig. 35, 1637 when $N_{\text {wind }}$ increases from 3 to 5 , an efficient trade-off be1638 tween the achievable throughput and the complexity imposed 1639 occurs, where the achievable throughput rapidly increases, if 1640 we invest more computational resources. By contrast, with $1641 N_{\text {wind }}=5$, the attainable throughput improvement becomes 1642 negligible upon increasing the affordable complexity.

\section{CONCLUSiOns AND Future Work}

\section{A. Summary and Conclusions}

1645 In this paper, we have surveyed noncoherent successive re1646 laying techniques for multi-user wireless systems, which are ca1647 pable of significantly improving the system's spectral efficiency 1648 by mitigating the half-duplex relaying-induced throughput loss. 1649 Furthermore, they are capable of circumventing the power- hungry channel estimation process at the cost of the typical $3 \mathrm{~dB} 1650$ power-loss. Hence, in the spirit of our discourse in Section I, the 1651 advocated solutions are capable of striking an attractive com- 1652 promise amongst the conflicting design factors, namely the ef- 1653 fective throughput, the attainable coding gain, the bit error 1654 ratio achieved and the computational complexity imposed. 1655

After briefly reciting the relevant history and detailing the 1656 general motivations behind successive relaying and noncoher- 1657 ent detection in Section I, we offered further insights into 1658 the advantages achieved by the SR regimes in Section II-A. 1659 Furthermore, the mathematical derivation of the state-of-the- 1660 art MSDSD algorithms was reviewed in Section II-C, D, E, 1661 respectively. These tutorial reflections introduced Section II, 1662 paving the way for discussing our more sophisticated NC-SR 1663 based prototypes.

In Section III-A and B, the successive AF relaying aided 1665 single-user DS-CDMA uplink was introduced, where the spe- 1666 cific DS-CDMA spreading-despreading strategy was high- 1667 lighted and the signals received at the DN were analysed. As a 1668 benefit of the diversity gain gleaned, this basic prototype system 1669 is capable of reducing the power-dissipation by $8 \mathrm{~dB}$ at the 1670 target BER of $10^{-4}$ compared to the classic "benchmark-II" 1671 of Table IX as shown in Section III-B. Consequently, the BER 1672 performance was improved at the cost of imposing a higher 1673 computational complexity on the system. Then, we further 1674 developed our prototype system from the single-user scenario 1675 to a more realistic multi-user scenario in Section III-C. In 1676 Section III-C1, we revealed the noise accumulation problem 1677 imposed by the IRI in our system, which was mitigated by a 1678 DS-CDMA based interference suppression regime as clarified 1679 in Section III-C2. As a theoretical contribution, the noncoherent 1680 DCMC capacity of the AF multi-user scenario based prototype 1681 system was derived in Section III-D. The DCMC capacity 1682 results of Fig. 26 indicated that the capacity of the AF based 1683 SRAN significantly exceeds that of conventional AF relaying 1684 or that of single-link direct-transmission, provided that RNs 1685 located at the appropriate positions are assigned. 1686

Finally, in Section IV-A, we introduced another prototype 1687 system, namely the successive DF relaying aided multi-user 1688 DS-CDMA uplink, which was developed from the basic pro- 1689 totype introduced in Section III-A for the sake of improving 1690 the system's energy efficiency. The NC DCMC capacity of 1691 the DF based SRAN embedded in the multi-user DS-CDMA 1692 uplink was quantified in Section IV-B. The related simulation 1693 results portrayed in Fig. 28 revealed that the DF based SRAN 1694 outperforms the AF based SRAN, especially in the low-SNR 1695 region ( $\mathrm{SNR}<0 \mathrm{~dB})$. However, this capacity improvement 1696 with respect to the AF based SRAN introduced in Section III-C 1697 was obtained at the cost of imposing increased complexity at 1698 the RN owing to replacing the AF based RN by the DF based 1699 RN. As a further advance, in Section IV-C, a relay-aided SISO- 1700 MSDSD assisted three-stage iterative transceiver was designed 1701 for efficiently implementing the proposed DF based SRAN. We 1702 observe in Fig. 33 that this transceiver architecture attains a 1703 performance, which is within about $2.6 \mathrm{~dB}$ of the DF-based 1704 SRAN's capacity. Again, the fact that the DF based SRAN is 1705 capable of achieving a higher energy efficiency than the AF 1706 based SRAN is illustrated in Fig. 33. However, it is important 1707 
TABLE XIII

CRITICAL TECHNiques In SuPPORT OF THE NC-SR Aided Multi-UsER WIRELESS SySTEMS INTRODUCED IN THIS TREATISE

\begin{tabular}{|l|r|}
\hline Advanced Techniques & The Sections Involved \\
\hline \hline Advanced NC detection algorithms & Sections II-C, II-D and II-E \\
\hline Interference suppression techniques & Sections III-A and III-C \\
\hline DCMC capacity based characterization & Sections III-D and IV-B \\
\hline Three-stage turbo decoder designs & Section IV-C \\
\hline EXIT-chart based analysis & Section IV-D \\
\hline
\end{tabular}

1708 to remember that this phenomenon relies on the precondition 1709 that we operate our SRAN in a weak-IRI scenario.

1710 The salient techniques employed in support of the advocated 1711 NC-SR aided multi-user systems are also briefly summarized in 1712 Table XIII.

\section{B. Design Guideline}

1716

1717

1718

1719

1720

1721

1722

1723

1724

1725

1726

1727

1728

1729

1730

1731

1732

1733

1734

1735

1736

1737

1738

1739

1740

1741

1742

1743

1744

1745

1746

1747

1748

1749

1750

1751

1752

1753

1754
- MIMO techniques have been invoked in support of the operational $3 \mathrm{G}$ or $4 \mathrm{G}$ wireless communication standards. However, a crucial condition to be satisfied for achieving the spatial diversity gain promised by the family of MIMO techniques is that the multiple co-located antenna elements have to be sufficiently far apart for the sake of experiencing independent fading. To circumvent this limitation, we can rely on the cooperative communication techniques, where the single-antenna-based mobiles, which are sufficiently far apart may form a VAA.

- However, the conventional three-terminal, two-phase based cooperative systems impose a severe 50\% multiplexing loss due to the half-duplex transmit/receive modes of practical contemporary transceivers.

- To recover the $50 \%$ throughput loss of half-duplex relaying, we may advocate the successive relaying regime of Section II-A. The main idea is to use a pair of half-duplex relays for mimicking a full-duplex relay.

- On the other hand, in support of a coherent cooperative system having a large number of propagation paths owing to employing multiple RNs, the estimation of the large number of channels involved will significantly increase the computational complexity, whilst imposing a high pilot overhead, especially at high normalized Doppler frequencies. Furthermore, it is somewhat unrealistic to expect that in addition to the task of relaying, the relay could altruistically afford to carry out the complex and power-hungry channel estimation of the source-to-relay link in support of coherent detection.

- Against this background, in the context of cooperative communication, we may propose the employment of noncoherent detection for the sake of operating without any requirement of channel estimation.

- Compared to the CDD or MSDD algorithms, the cluster of MSDSD algorithms strikes an attractive trade-off between the BER performance attained and the complexity imposed, which may be considered as a state-of-the-art family of noncoherent detection techniques.

- Given the above-mentioned advantages of successive relaying and the associated MSDSD algorithms, we may beneficially combine them for the sake of constituting an attractive solution, which is capable of circumvent- 1757 ing both the $50 \%$ throughput loss and the power-hungry 1758 channel estimation. Then, to suppress the successive re- 1759 laying induced interferences, we may additionally invoke 1760 the classic DS-CDMA technique. Finally, we arrive at 1761 the successive AF relaying aided single-user DS-CDMA 1762 uplink advocated in Section III-A.

- For the sake of characterizing the impact of diverse 1764 sources of interference (MAI, CCI, IRI), we may then ex- 1765 tend the communication scenario considered to multi-user 1766 scenarios. We may use the noncoherent DCMC capacity 1767 bound of the resulting system for quantifying its benefits 1768 over its conventional counterparts.

- In contrast to the AF protocol, the DF protocol may 1770 achieve a better BER performance, since it is capable 1771 of supporting the employment of sophisticated coding 1772 schemes. Correspondingly, we designed a sophisticated 1773 relay-aided SISO-MSDSD assisted three-stage iterative- 1774 detection based transceiver architecture in Section IV-C, 1775 which is capable of striking an attractive trade-off be- 1776 tween the BER performance attained and the complexity 1777 imposed.

\section{Future Research}

There remain numerous challenging problems associated 1780 with the design of NC-SR based wireless communication sys- 1781 tems, which need further investigation in the future:

1) The successive relaying induced interference may be 1784 mitigated by the classic DS-CDMA technique, despite 1785 dispensing with any CSI. However, this is achieved at 1786 the expense of a potential user-load reduction for the 1787 CDMA system, since each relaying link requires its own 1788 unique spreading sequence, rather than relying on a single 1789 spreading code. Alternatively, it is possible to suppress 1790 the interference in a noncoherent communication system 1791 without requiring any extra orthogonal channel resources. 1792 This may be realized with the aid of the differential 1793 interference suppression (DIS) philosophy of [92]. In 1794 particular, a novel amalgam of the adaptive modified 1795 Newton algorithm [93] and the SISO-MSDSD algorithm 1796 may constitute a powerful state-of-the-art DIS regime. 1797 Therefore, we may use the DIS regime for suppressing 1798 the successive relaying induced interference.

2) As reported in [94]-[97], the performance of the entire 1800 network, especially its DMT can be further improved 1801 by allowing more users and relays to join in the co- 1802 operation. Inspired by this result, it may be beneficial 1803 to further extend the NC-SR based prototype system of 1804 Section III-A to the more sophisticated multi-user multi- 1805 relay scenarios. Correspondingly, an appropriate solution 1806 for efficiently organizing the multiple nodes of a large- 1807 scale cooperative wireless network is desired. For the 1808 sake of satisfying this demand, we may employ the 1809 family of adaptive network coding techniques detailed 1810 in [98]-[100]. Consequently, we may create an adaptive 1811 network coded successive relaying regime, where an im- 1812 proved DMT can be expected. 
TABLE XIV

GLOSSARY

\begin{tabular}{|c|c|}
\hline $\mathrm{AF}$ & amplify-and-forward \\
\hline AN & accumulated noise \\
\hline AWGN & additive white Gaussian noise \\
\hline BER & bit-error-rate \\
\hline BS & base station \\
\hline CCI & co-channel interference \\
\hline CSI & channel state information \\
\hline CIR & channel impulse response \\
\hline CDD & conventional differential detection \\
\hline $\mathrm{CR}$ & conventional relaying \\
\hline CCL & cross-correlation \\
\hline DF & decode-and-forward \\
\hline DN & destination node \\
\hline DT & direct-transmission \\
\hline DM & differential modulator \\
\hline DMT & diversity-multiplexing trade-off \\
\hline DIS & differential interference suppression \\
\hline DF-DD & decision-feedback aided differential detection \\
\hline DS-CDMA & direct-sequence code-decision multiple-access \\
\hline EXIT & extrinsic information transfer \\
\hline FD & full-duplex \\
\hline FNC & Frobenius norm calculation \\
\hline FDR & full-duplex relaying \\
\hline Fano-MSDD & MSDD based Fano-algorithm \\
\hline HD & half-duplex \\
\hline HDB & hard-decision-based \\
\hline IRI & inter-relay interference \\
\hline ISR & IRI to signal power ratio \\
\hline LLR & log-likelihood ratio \\
\hline MS & mobile station \\
\hline MAI & multiple-access interference \\
\hline MIMO & Multiple-Input Multiple-Output \\
\hline MSDD & multiple-symbol differential detection \\
\hline MPSK & $M$-ary phase-shift keying \\
\hline MSDSD & multiple-symbol differential sphere detection \\
\hline$M_{c}$-DPSK & $M_{c}$-ary differential phase-shift keying \\
\hline $\mathrm{NC}$ & non-coherent \\
\hline NC-SR & non-coherent successive relaying \\
\hline PN & pseudo-noise \\
\hline PDF & probability density function \\
\hline RN & relay node \\
\hline RSC & recursive systematic convolutional code \\
\hline RMO & real-valued multiplication operation \\
\hline SL & single-link \\
\hline $\mathrm{SN}$ & source node \\
\hline SI & self-interference \\
\hline SR & successive relaying \\
\hline SF & spreading factor \\
\hline SDB & soft-decision-based \\
\hline SRAN & successive relaying aided network \\
\hline SIMO & single-input-multiple-output \\
\hline SISO & single-input single-output \\
\hline SISO-MSDSD & soft-input soft-output MSDSD \\
\hline URC & unity-rate-code \\
\hline VAA & virtual antenna array \\
\hline
\end{tabular}

3) It has been demonstrated in numerous studies [16], [101] that an appropriate resource allocation is capable of significantly improving the energy efficiency and/or spectral efficiency of cooperative communications. Hence it is desirable to carry out the investigation of the advanced optimal power allocation and optimal rate allocation [102] regimes in the context of our NC successive relaying aided system.

\section{APPENDIX}

Glossary see Table XIV.

\section{REFERENCES}

[1] D. C. Kilper et al., "Power trends in communication networks," 1826 IEEE J. Sel. Topics Quantum Electron., vol. 17, no. 2, pp. 275-284, 1827 Mar./Apr. 2011.

[2] S. M. Alamouti, "A simple transmit diversity technique for wireless 1829 communications," IEEE J. Sel. Areas Commun., vol. 16, no. 8, pp. 1451- 1830 1458, Oct. 1998.

1831

[3] I. E. Telatar, "Capacity of multi-antenna Gaussian channels," Eur. Trans. 1832 Telecommun., vol. 10, pp. 585-595, Nov. 1999.

1833

[4] G. Foschini, G. Golden, R. Valenzuela, and P. Wolniansky, "Simplified 1834 processing for high spectral efficiency wireless communication employ- 1835 ing multi-element arrays," IEEE J. Sel. Areas Commun., vol. 17, no. 11, 1836 pp. 1841-1852, Nov. 1999

1837

[5] L. Zheng and D. N. C. Tse, "Diversity and multiplexing: A fundamental 1838 tradeoff in multiple-antenna channels," IEEE Trans. Inf. Theory, vol. 49, 1839 no. 5, pp. 1073-1096, May 2003.

1840

[6] A. J. Paulraj, D. A. Gore, R. U. Nabar, and H. Bölcskei, "An overview 1841 of MIMO communications-A key to gigabit wireless," Proc. IEEE, 1842 vol. 92, no. 2, pp. 198-218, Feb. 2004.

[7] C. Dubuc, D. Starks, T. Creasy, and Y. Hou, "A MIMO-OFDM prototype 1844 for next-generation wireless WANs," IEEE Commun. Mag., vol. 42, 1845 no. 12 , pp. 82-87, Dec. 2004

1846

[8] L. Hanzo, O. R. Alamri, M. El-Hajjar, and N. Wu, Near-Capacity 1847 Multi-Functional MIMO Systems: Sphere-Packing, Iterative Detection 1848 and Cooperation. New York, NY, USA: Wiley, 2009, ch. 9.

[9] E. C. Van der Meulen, "Three-terminal communication channels," Adv. 1850 Appl. Probability, vol. 3, no. 1, pp. 120-154, 1971.

[10] T. M. Cover and A. A. El Gamal, "Capacity theorems for the relay chan- 1852 nel," IEEE Trans. Inf. Theory, vol. 25, no. 5, pp. 572-584, Sep. 1979.1853

[11] J. N. Laneman, D. N. C. Tse, and G. W. Wornell, "Cooperative diversity 1854 in wireless networks: Efficient protocols and outage behavior," IEEE 1855 Trans. Inf. Theory, vol. 50, no. 12, pp. 3062-3080, Dec. 2004.

[12] J. N. Laneman and G. W. Wornell, "Distributed space-time-coded pro- 1857 tocols for exploiting cooperative diversity in wireless networks," IEEE 1858 Trans. Inf. Theory, vol. 49, no. 10, pp. 2415-2425, Oct. 2003.

[13] A. Sendonaris, E. Erkip, and B. Aazhang, "User cooperation diversity- 1860 part I: System description," IEEE Trans. Commun., vol. 51, no. 11, 1861 pp. 1927-1938, Nov. 2003.

1862

[14] A. Sendonaris, E. Erkip, and B. Aazhang, "User cooperation diversity- 1863 Part II: Implementation aspects and performance analysis," IEEE Trans. 1864 Commun., vol. 51, no. 11, pp. 1939-1948, Nov. 2003. 1865

[15] K. Azarian, H. E. Gamal, and P. Schniter, "On the achievable diversity- 1866 multiplexing tradeoff in half-duplex cooperative channels," IEEE Trans. 1867 Inf. Theory, vol. 51, no. 12, pp. 4152-4172, Dec. 2005.

1868

[16] A. Høst-Madsen and J. Zhang, "Capacity bounds and power allocation 1869 for wireless relay channels," IEEE Trans. Inf. Theory, vol. 51, no. 6, 1870 pp. 2020-2040, Jun. 2005.

[17] A. Bletsas, A. Khisti, D. P. Reed, and A. Lippman, "A simple cooperative 1872 diversity method based on network path selection," IEEE J. Sel. Areas 1873 Commun., vol. 24, no. 3, pp. 659-672, Mar. 2006.

1874

[18] G. Kramer, M. Gastpar, and P. Gupta, "Cooperative strategies and capac- 1875 ity theorems for relay networks," IEEE Trans. Inf. Theory, vol. 51, no. 9, 1876 pp. 3037-3063, Sep. 2005.

1877

[19] J. G. Andrews, H. Claussen, M. Dohler, S. Rangan, and M. C. Reed, 1878 "Femtocells: Past, present, and future," IEEE J. Sel. Areas Commun., 1879 vol. 30, no. 3, pp. 497-508, Apr. 2012.

[20] R. U. Nabar, H. Bölcskei, and F. W. Kneubühler, "Fading relay channels: 1881 Performance limits and space-time signal design,” IEEE J. Sel. Areas 1882 Commun., vol. 22, no. 6, pp. 1099-1109, Aug. 2004.

1883

[21] H. Suzuki, K. Itoh, Y. Ebine, and M. Sato, "A booster configuration with 1884 adaptive reduction of transmitter-receiver antenna coupling for pager sys- 1885 tems," in Proc. IEEE 50th Veh. Technol. Conf., Sep. 1999, pp. 1516-1520. 1886

[22] H. Sakai, T. Oka, and K. Hayashi, "A simple adaptive filter method for 1887 cancellation of coupling wave in OFDM signals at SFN relay station," in 1888 Proc. 14th EUSIPCO, Sep. 2006, pp. 1-5.

1889

[23] K. M. Nasr, J. P. Cosmas, M. Bard, and J. Gledhill, "Performance of an 1890 echo canceller and channel estimator for on-channel repeaters in DVB- 1891 T/H networks," IEEE Trans. Broadcast., vol. 53, no. 3, pp. 609-618, 1892 Sep. 2007.

[24] H. Ju, E. Oh, and D. Hong, "Improving efficiency of resource us- 1894 age in two-hop full duplex relay systems based on resource sharing 1895 and interference cancellation," IEEE Trans. Wireless Commun., vol. 8, 1896 no. 8, pp. 3933-3938, Aug. 2009.

[25] J. Sangiamwong, T. Asai, J. Hagiwara, Y. Okumura, and T. Ohya, "Joint 1898 multi-filter design for full-duplex MU-MIMO relaying," in Proc. IEEE 1899 69th Veh. Technol. Conf., Apr. 2009, pp. 1-5. 
[26] T. Riihonen, S. Werner, and R. Wichman, "Mitigation of loopback selfinterference in full-duplex MIMO relays," IEEE Trans. Signal Process., vol. 59, no. 12, pp. 5983-5993, Dec. 2011.

[27] J. I. Choi, M. Jain, K. Srinivasan, P. Levis, and S. Katti, "Achieving single channel, full duplex wireless communication," in Proc. 16th Annu. Int. Conf. Mobile Comput. Netw., 2010, pp. 1-12.

[28] M. Jain et al., "Practical, real-time, full duplex wireless," in Proc. 17th Annu. Int. Conf. Mobile Comput. Netw., 2011, pp. 301-312.

[29] D. Bharadia, E. McMilin, and S. Katti, "Full duplex radios," in Proc. ACM SIGCOMM, 2013, pp. 375-386.

[30] T. Riihonen, S. Werner, and R. Wichman, "Comparison of full-duplex and half-duplex modes with a fixed amplify-and-forward relay," in Proc. IEEE Wireless Commun. Netw. Conf., Apr. 2009, pp. 1-5.

[31] T. Riihonen, S. Werner, and R. Wichman, "Hybrid full-duplex/halfduplex relaying with transmit power adaptation," IEEE Trans. Wireless Commun., vol. 10, no. 9, pp. 3074-3085, Sep. 2011.

[32] B. P. Day, A. R. Margetts, D. W. Bliss, and P. Schniter, "Full-duplex MIMO relaying: Achievable rates under limited dynamic range," IEEE J. Sel. Commun., vol. 30, no. 8, pp. 1541-1553, Sep. 2012.

33] B. Rankov and A. Wittneben, "Spectral efficient signaling for halfduplex relay channels," in Proc. Conf. Rec. 39th Asilomar Conf. Signals, Syst. Comput., 2005, pp. 1066-1071.

[34] B. Rankov and A. Wittneben, "Spectral efficient protocols for halfduplex fading relay channels," IEEE J. Sel. Areas Commun., vol. 25, no. 2, pp. 379-389, Feb. 2007.

[35] P. Popovski and H. Yomo, "Physical network coding in two-way wireless relay channels," in Proc. IEEE Int. Conf. Commun., Jun. 2007, pp. 707-712.

[36] C. Hausl and J. Hagenauer, "Iterative network and channel decoding for the two-way relay channel," in Proc. IEEE Int. Conf. Commun., Jun. 2006, vol. 4, pp. 1568-1573.

37] R. Zhang, Y. C. Liang, C. C. Chai, and S. G. Cui, "Optimal beamforming for two-way multi-antenna relay channel with analogue network coding," IEEE J. Sel. Areas Commun., vol. 27, no. 5, pp. 699-712, Jun. 2009.

[38] R. H. Y. Louie, Y. Li, and B. Vucetic, "Practical physical layer network coding for two-way relay channels: Performance analysis and comparison," IEEE Trans. Wireless Commun., vol. 9, no. 2, pp. 764-777, Feb. 2010.

39] W. Chen, L. Hanzo, and Z. G. Cao, "Network coded modulation for twoway relaying," in Proc. IEEE Wireless Commun. Netw. Conf., Mar. 2011, pp. 1765-1770.

[40] R. Wang and M. Tao, "Joint source and relay precoding designs for MIMO two-way relaying based on MSE criterion," IEEE Trans. Signal Process., vol. 60, no. 3, pp. 1352-1365, Mar. 2012.

[41] P. Popovski and H. Yomo, "Bi-directional amplification of throughput in a wireless multi-hop network," in Proc. IEEE 63rd Veh. Technol. Conf., May 2006, vol. 2, pp. 588-593.

42] L. Xiao, T. Fuja, J. Kliewer, and D. Costello, "Nested codes with multiple interpretations," in Proc. 40th Annu. Conf. Inf. Sci. Syst., Mar. 2006, pp. 851-856.

[43] T. Oechtering and A. Sezgin, "A new cooperative transmission scheme using the space-time delay code," in Proc. ITG Workshop Smart Antennas, Mar. 2004, pp. 41-48.

[44] S. Yang and J. C. Belfiore, "On slotted amplify-and-forward cooperative diversity schemes," in Proc. IEEE Int. Symp. Inf. Theory, pp. 2446-2450, Jul. 2006.

[45] S. Yang and J. C. Belfiore, "Towards the optimal amplify-and-forward cooperative diversity scheme," IEEE Trans. Inf. Theory, vol. 53, no. 9, pp. 3114-3126, Sep. 2007.

[46] Y. J. Fan, C. Wang, J. S. Thompson, and H. V. Poor, "Recovering multiplexing loss through successive relaying using repetition coding," IEEE Trans. Commun., vol. 6, no. 12, pp. 4484-4493, Dec. 2007.

[47] C. B. Luo, Y. Gong, and F.-C. Zheng, "Interference cancellation in twopath successive relay system with network coding," in Proc. IEEE 21st Int. Symp. Pers. Indoor Mobile Radio Commun., Sep. 2010, pp. 465-469.

[48] C. B. Luo, Y. Gong, and F.-C. Zheng, "Full interference cancellation for two-path relay cooperative networks," IEEE Trans. Veh. Technol., vol. 60, no. 1, pp. 343-347, Jan. 2011.

[49] H. Wicaksana, S. H. Ting, C. K. Ho, W. H. Chin, and Y. L. Guan, "AF two-path half duplex relaying with inter-relay self interference cancellation: Diversity analysis and its improvement," IEEE Trans. Wireless Commun., vol. 8, no. 9, pp. 4720-4729, Sep. 2009.

[50] F. Tian, W. Zhang, W.-K. Ma, P. C. Ching, and H. V. Poor, "An effective distributed space-time code for two-path successive relay networks," IEEE Trans. Commun., vol. 59, no. 8, pp. 2254-2263, Aug. 2011.

[51] E. Başar, U. Aygölü, E. Panayırcı, and H. V. Poor, "A reliable successive relaying protocol," IEEE Trans. Commun., vol. 62, no. 5, pp. 1431-1443, May 2014.
[52] C. I. Bang and M. H. Lee, "An analysis of pilot symbol assisted 16 QAM 1979 in the Rayleigh fading channel," IEEE Trans. Consum. Electron., vol. 41, 1980 no. 4, pp. 1138-1141, Nov. 1995.

1981

[53] D. Divsalar and M. K. Simon, "Multiple symbol differential detec- 1982 tion of MPSK," IEEE Trans. Commun., vol. 38, no. 3, pp. 300-308, 1983 Mar. 1990.

[54] K. Mackenthun, "A fast algorithm for multiple-symbol differen- 1985 tial detection of MPSK," IEEE Trans. Commun., vol. 42, no. 234, 1986 pp. 1471-1474, Apr. 1994.

1987

[55] H. Leib and S. Pasupathy, "The phase of a vector perturbed by Gaussian 1988 noise and differentially coherent receivers," IEEE Trans. Inf. Theory, 1989 vol. 34, no. 6, pp. 1491-1501, Nov. 1988.

[56] F. Edbauer, "Bit error rate of binary and quaternary DPSK signals with 1991 multiple differential feedback detection," IEEE Trans. Commun., vol. 40, 1992 no. 3, pp. 457-460, Mar. 1992.

[57] F. Adachi and M. Sawahashi, "Decision feedback multiple-symbol dif- 1994 ferential detection for M-ary DPSK," Electron. Lett., vol. 29, no. 15, 1995 pp. 1385-1387, Jul. 1993.

1996

[58] D. Divsalar and M. K. Simon, "Maximum-likelihood differential de- 1997 tection of uncoded and trellis coded amplitude phase modulation over 1998 AWGN and fading channels-metrics and performance," IEEE Trans. 1999 Commun., vol. 42, no. 1, pp. 76-89, Jan. 1994.

[59] P. Ho and D. Fung, "Error performance of multiple-symbol differential 2001 detection of PSK signals transmitted over correlated Rayleigh fading 2002 channels," IEEE Trans. Commun., vol. 40, no. 10, pp. 1566-1569, 2003 Oct. 1992.

[60] R. Schober, W. H. Gerstacker, and J. B. Huber, "Decision-feedback 2005 differential detection of MDPSK for flat rayleigh fading channels," IEEE 2006 Trans. Commun., vol. 47, no. 7, pp. 1025-1035, Jul. 1999.

[61] O. Damen, H. E. Gamal, and G. Gaire, "On maximum likelihood detec- 2008 tion and the search for the closest lattice point," IEEE Trans. Inf. Theory, 2009 vol. 49, no. 10, pp. 2389-2402, Oct. 2003.

2010

[62] L. Lampe, R. Schober, V. Pauli, and C. Windpassinger, "Multiple- 2011 symbol differential sphere decoding," IEEE Trans. Commun., vol. 53, 2012 no. 12, pp. 1981-1985, Dec. 2005.

2013

[63] P. Pun and P. Ho, "The performance of Fano-multiple symbol dif- 2014 ferential detection," in Proc. IEEE Int. Conf. Commun., May 2005, 2015 pp. $2516-2521$

2016

[64] P. Pun and P. Ho "Fano multiple-symbol differential detectors for differ- 2017 ential unitary space-time modulation," IEEE Trans. Commun., vol. 55, 2018 no. 3, pp. 540-550, Mar. 2007.

[65] R. M. Fano, "A heuristic discussion of probabilistic decoding," IEEE 2020 Trans. Inf. Theory, vol. 9, no. 2, pp. 64-74, Apr. 1963.

[66] V. Pauli, L. Lampe, and R. Schober, "Turbo DPSK using soft multiple- 2022 symbol differential sphere decoding," IEEE Trans. Inf. Theory, vol. 52, 2023 no. 4, pp. 1385-1398, Apr. 2006.

2024

[67] L. Wang and L. Hanzo, "The amplify-and-forward cooperative up- 2025 link using multiple-symbol differential sphere-detection," IEEE Signal 2026 Process. Lett., vol. 16, no. 10, pp. 913-916, Oct. 2009.

2027

[68] L. Wang and L. Hanzo, "Multiple-symbol differential sphere detection 2028 for the amplify-and-forward cooperative uplink," in Proc. IEEE 70th 2029 Veh. Technol. Conf.—Fall, Sep. 2009, pp. 1-5.

[69] L. Li and L. Hanzo, "Multiple-symbol differential sphere detection aided 2031 successive relaying in the cooperative DS-CDMA uplink," in Proc. IEEE 2032 Wireless Commun. Netw. Conf., Mar. 2011, pp. 1875-1880. 2033

[70] L. Li, L. Wang, and L. Hanzo, "The capacity of successive DF relaying 2034 and using soft multiple-symbol differential sphere detection," in Proc. 2035 IEEE Global Commun. Conf., Dec. 2011, pp. 1-5.

2036

[71] L. Hanzo, Y. Akhtman, M. Jiang, and L. Wang, MIMO-OFDM for LTE, 2037 WIFI and WIMAX: Coherent versus Non-Coherent and Cooperative 2038 Turbo-Transceivers. Hoboken, NJ, USA: Wiley, 2010.

[72] H. Van Trees, Detection, Estimation and Modulation Theory: Part I, 2040 vol. 6. New York, NY, USA: Wiley, Mar. 1968.

2041

[73] D. Divsalar, M. K. Simon, and M. Shahshahani, "The performance of 2042 trellis-coded MDPSK with multiple symbol detection," IEEE Trans. 2043 Commun., vol. 38, no. 9, pp. 1391-1403, Sep. 1990.

[74] W. C. Lindsey and M. K. Simon, Telecommunication Systems Engineer- 2045 ing. Englewood Cliffs, NJ, USA: Prentice-Hall, 1973.

2046

[75] L. Hanzo, L.-L. Yang, E.-L. Kuan, and K. Yen, Single and Multi-Carrier 2047 DS-CDMA: Multi-User Detection, Space-Time Spreading, Synchronisa- 2048 tion and Standards. New York, NY, USA: Wiley, 2005.

[76] E. Agrell, T. Eriksson, A. Vardy, and K. Zeger, "Closest point search 2050 in lattices," IEEE Trans. Inf. Theory, vol. 48, no. 8, pp. 2201-2214, 2051 Aug. 2002.

2052

[77] W. Fang, L.-L. Yang, and L. Hanzo, "Single-user performance of 2053 direct-sequence code-division multiple-access using relay diversity 2054 and power allocation," IET Commun., vol. 2, no. 3, pp. 462-472, 2055 Mar. 2008. 
[78] S. Won, K. Lee, and L. Hanzo, "Initial code acquisition in the cooperative noncoherent MIMO DS-CDMA downlink," IEEE Trans. Veh. Technol., vol. 58, no. 3, pp. 1387-1395, Mar. 2009.

79] A. Abrardo, "Noncoherent MLSE detection of M-DPSK for DS-CDMA wireless systems," IEEE Trans. Veh. Technol., vol. 52, no. 6, pp. 14351446, Nov. 2003.

[80] T. S. Rappaport, Wireless Communications: Principles and Practice. Upper Saddle River, NJ, USA: Prentice-Hall, 1996.

81] L. Li, L. Wang, and L. Hanzo, "Successive AF/DF relaying in the cooperative DS-CDMA uplink: Capacity analysis and its system architecture," IEEE Trans. Veh. Technol., vol. 62, no. 2, pp. 655-666, Feb. 2013

82] L. Li, L. Wang, and L. Hanzo, "Capacity analysis of the successive AF relaying aided cooperative DS-CDMA uplink," in Proc. IEEE Wireless Commun. Netw. Conf., Apr. 2012, pp. 3057-3062.

83] Y. Zhao, R. Adve, and T. J. Lim, "Improving amplify-and-forward relay networks: Optimal power allocation versus selection," IEEE Trans. Wireless Commun., vol. 6, no. 8, pp. 3114-3123, Aug. 2007.

84] Y. W. Ding, J. K. Zhang, and K. M. Wong, "Ergodic channel capacity for the amplify-and-forward half-duplex cooperative systems," IEEE Trans. Inf. Theory, vol. 55, no. 2, pp. 713-730, Feb. 2009.

85] L. K. Kong, S. X. Ng, R. G. Maunder, and L. Hanzo, "Near-capacity cooperative space-time coding employing irregular design and successive relaying," IEEE Trans. Commun., vol. 58, no. 2, pp. 2232-2241, Aug. 2010.

86] R. Schober and L. Lampe, "Noncoherent receivers for differential spacetime modulation," IEEE Trans. Commun., vol. 50, no. 5, pp. 768-777, May 2002.

87] Y. B. Liang and V. V. Veeravalli, "Capacity of noncoherent time-selective block rayleigh flat-fading channel," in Proc. IEEE Int. Symp. Inf. Theory, 2002, p. 166.

[88] L. Wang, L. K. Kong, S. X. Ng, and L. Hanzo, "Code-rate-optimized differentially modulated near-capacity cooperation," IEEE Trans. Commun., vol. 59, no. 8, pp. 2185-2195, Aug. 2011.

89] A. Ashikhmin, G. Kramer, and S. ten Brink, "Extrinsic information transfer functions: Model and erasure channel properties," IEEE Trans. Inf. Theory, vol. 50, no. 11, pp. 2657-2673, Nov. 2004.

[90] B. Zhao and M. C. Valenti, "Distributed turbo coded diversity for relay channel," Electron. Lett., vol. 39, no. 10, pp. 786-787, May 2003.

91] L. K. Kong, S. X. Ng, R. G. Maunder, and L. Hanzo, "Irregular distributed space-time code design for near-capacity cooperative communications," in Proc. IEEE Veh. Technol. Conf., Sep. 2009, vol. 1, pp. 1-6.

92] S. K. Cheung and R. Schober, "Differential spatial multiplexing," IEEE Trans. Wireless Commun., vol. 5, no. 8, pp. 2127-2135, Aug. 2006.

[93] J. Yang, F. Yang, H. S. Xi, and W. Guo, "Robust adaptive modified newton algorithm for generalized eigende composition and its application," EURASIP J. Adv. Signal Process., vol. 2007, no. 2, pp. 1-10, Jun. 2007.

94] J. Kim, D. S. Michalopoulos, and R. Schober, "Diversity analysis of multi-user multi-relay networks," IEEE Trans. Wireless Commun., vol. 10, no. 7, pp. 2380-2389, Jul. 2011.

95] C. Wang, Y. J. Fan, J. S. Thompson, and H. V. Poor, "A comprehensive study of repetition-coded protocols in multi-user multi-relay networks," IEEE Trans. Wireless Commun., vol. 8, no. 8, pp. 4329-4339, Aug. 2009.

[96] S. Chen, W. Wang, and X. Zhang, "Performance analysis of multiuser diversity in cooperative multi-relay networks under rayleigh-fading channels," IEEE Trans. Wireless Commun., vol. 8, no. 7, pp. 3415-3419, Jul. 2009.

97] I. Krikidis, J. S. Thompson, S. McLaughlin, and N. Goertz, "Max-min relay selection for legacy amplify-and-forward systems with interference," IEEE Trans. Wireless Commun., vol. 8, no. 6, pp. 3016-3027, Jun. 2009.

98] X. Bao and J. Li, "Adaptive Network Coded Cooperation (ANCC) for wireless relay networks: Matching code-on-graph with network-ongraph," IEEE Trans. Wireless Commun., vol. 7, no. 2, pp. 574-583, Feb. 2008.

99] M. Xiao and M. Skoglund, "Multiple-user cooperative communications based on linear network coding," IEEE Trans. Commun., vol. 58, no. 12, pp. 3345-3351, Dec. 2010.

00] J. L. Rebelatto, B. F. Uchôa-Filho, Y. Li, and B. Vucetic, "Multiuser cooperative diversity through network coding based on classical coding theory," IEEE Trans. Signal Process., vol. 60, no. 2, pp. 916-926, Feb. 2012.

01] J. W. Huang, Z. Han, M. Chiang, and H. V. Poor, "Auction-based resource allocation for cooperative communications," IEEE J. Sel. Areas Commun., vol. 26, no. 7, pp. 1226-1237, Sep. 2008.

02] C. Y. Ng, C.-W. Sung, and K. W. Shum, "Rate allocation for cooperative transmission in parallel channels," in Proc. IEEE Global Commun. Conf., 2007, pp. 3921-3925.

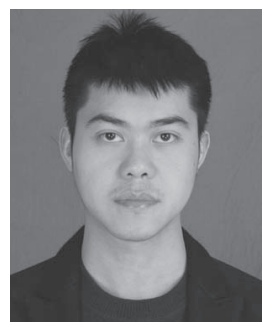

Li Li received the Ph.D. degree from Southampton 2135 Wireless (SW) Group, School of Electronics and 2136 Computer Science, University of Southampton, in 2137 October 2013. Upon the completion of the Ph.D. 2138 degree, he conducted research as a Senior Research 2139 Assistant in the School of Electronics and Com- 2140 puter Science at the University of Southampton from 2141 December 2013 to December 2014, where he par- 2142 ticipated in the European Union Concerto project. 2143 In January 2015, he joined the Provincial Key Lab 2144 of Information Coding and Transmission, Southwest 2145 Jiaotong University, Chengdu, China, serving as a Lecturer.

His research interests include channel coding, iterative detection, non- 2147 coherent transmission technologies, cooperative communications and network 2148 coding.

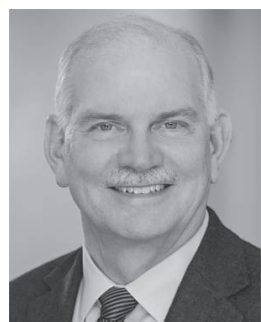

H. Vincent Poor (S'72-M'77-SM'82-F'87) re- 2150 ceived the Ph.D. degree in EECS from Princeton 215 University in 1977. From 1977 until 1990, he was 2152 on the faculty of the University of Illinois at Urbana- 2153 Champaign. Since 1990, he has been on the faculty 2154 at Princeton, where he is the Michael Henry Strater 2155 University Professor and Dean of the School of 2156 Engineering and Applied Science. He has also held 2157 visiting appointments at several universities, includ- 2158 ing most recently at Stanford and Imperial College. 2159 Dr. Poor's research interests are in the area of wire- 2160 less networks and related fields. Among his publications in these areas is 2161 the recent book, Mechanisms and Games for Dynamic Spectrum Allocation 2162 (Cambridge University Press, 2014).

Dr. Poor is a member of the U. S. National Academy of Engineering and 2164 the U. S. National Academy of Sciences, and is a foreign member of Academia 2165 Europaea and the Royal Society. He is also a Fellow of the American Academy 2166 of Arts and Sciences, the Royal Academy of Engineering (U.K.), and the 2167 Royal Society of Edinburgh. He received the Marconi and Armstrong Awards 2168 of the IEEE Communications Society in 2007 and 2009, respectively. Recent 2169 recognition of his work includes the 2014 URSI Booker Gold Medal, and 2170 honorary doctorates from several universities in Asia and Europe.

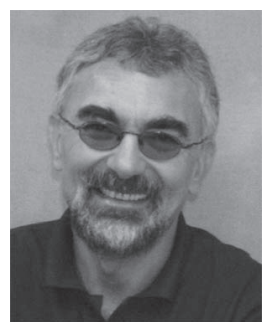

Lajos Hanzo received the degree in electronics in 2172 1976 and his doctorate in 1983. In 2009, he was 2173 awarded the honorary doctorate "Doctor Honoris 2174 Causa" by the Technical University of Budapest. 2175 During his 35-year career in telecommunications he 2176 has held various research and academic posts in 2177 Hungary, Germany and the U.K. Since 1986, he has 2178 been with the School of Electronics and Computer 2179 Science, University of Southampton, U.K., where he 2180 holds the chair in telecommunications. He has suc- 2181 cessfully supervised $80 \mathrm{Ph} . \mathrm{D}$. students, co-authored 2182 20 John Wiley/IEEE Press books on mobile radio communications totalling in 2183 excess of 10000 pages, published 1300 research entries at IEEE Xplore, acted 2184 both as TPC and General Chair of IEEE conferences, presented keynote lectures 2185 and has been awarded a number of distinctions. Currently, he is directing a 2186 100 -strong academic research team, working on a range of research projects 2187 in the field of wireless multimedia communications sponsored by industry, 2188 the Engineering and Physical Sciences Research Council (EPSRC), U.K., the 2189 European IST Programme and the Mobile Virtual Centre of Excellence (VCE), 2190 U.K. He is an enthusiastic supporter of industrial and academic liaison and 2191 he offers a range of industrial courses. He is also a Governor of the IEEE 2192 VTS. During 2008-2012, he was the Editor-in-Chief of the IEEE Press and a 2193 Chaired Professor also at Tsinghua University, Beijing. His research is funded 2194 by the European Research Council's Senior Research Fellow Grant. For further 2195 information on research in progress and associated publications please refer to 2196 http://www-mobile.ecs.soton.ac.uk. 


\section{AUTHOR QUERIES}

\section{AUTHOR PLEASE ANSWER ALL QUERIES}

AQ1 = Please provide keywords.

AQ2 = Table VI was cited only in Fig. 12 caption. Please provide citation in text.

AQ3 = Table VII was cited only in Fig. 15 caption. Please provide citation in text.

\section{END OF ALL QUERIES}

